\title{
Transfer of heart rate feedback training to reduce heart rate response to laboratory tasks
}

\author{
Jeffrey Louis Goodie \\ West Virginia University
}

Follow this and additional works at: https://researchrepository.wvu.edu/etd

\section{Recommended Citation}

Goodie, Jeffrey Louis, "Transfer of heart rate feedback training to reduce heart rate response to laboratory tasks" (2001). Graduate Theses, Dissertations, and Problem Reports. 1449.

https://researchrepository.wvu.edu/etd/1449

This Dissertation is protected by copyright and/or related rights. It has been brought to you by the The Research Repository @ WVU with permission from the rights-holder(s). You are free to use this Dissertation in any way that is permitted by the copyright and related rights legislation that applies to your use. For other uses you must obtain permission from the rights-holder(s) directly, unless additional rights are indicated by a Creative Commons license in the record and/ or on the work itself. This Dissertation has been accepted for inclusion in WVU Graduate Theses, Dissertations, and Problem Reports collection by an authorized administrator of The Research Repository @ WVU.

For more information, please contact researchrepository@mail.wvu.edu. 
Transfer of Heart Rate Feedback Training to Reduce Heart Rate Response to Laboratory Tasks

\author{
Jeffrey L. Goodie
}

Dissertation submitted to the Eberly College of Arts and Sciences at West Virginia University in partial fulfillment of the requirements of the degree of

\author{
Doctor of Philosophy \\ in \\ Clinical Psychology
}

\author{
Kevin Larkin, Ph.D., Chair \\ Marty Boone, Ph.D. \\ Barry Edelstein, Ph.D. \\ David Schaal, Ph.D. \\ Jeannie Sperry, Ph.D.
}

Department of Psychology

Morgantown, West Virginia

2001

Keywords: heart rate, feedback, cardiovascular reactivity

Copyright 2001 Jeffrey L. Goodie 


\author{
Abstract \\ Transfer of Heart Rate Feedback Training to Reduce \\ Heart Rate Response to Laboratory Tasks
}

\title{
By Jeffrey L. Goodie
}

Researchers have demonstrated that individuals can successfully reduce their heart rate (HR) response to a stressor when provided with heart rate feedback. However, it is unclear whether individuals can transfer HR reduction skills to stressors not used during HR feedback training. The present study used a multiple baseline, single subject design to examine the transfer of HR feedback training among six individuals. Participants were provided with HR feedback training during the presentation of a videogame, a mental arithmetic challenge, and a hand grip task until the participants reduced their HR to within $4 \mathrm{bpm}$ of their resting HR or until they completed three 2- hour training sessions. The participants' ability to reduce HR responses to the three training tasks with no HR feedback was assessed during an immediate post-training period, which followed training on each task. The participants' ability to reduce HR responses to the training tasks and a speech task was assessed during short delay (i.e., 1-2 days) and long delay (1-2 weeks) post-training sessions. Overall, participants demonstrated that during HR feedback training, they could successfully reduce their HR and generally could maintain this reduction in HR to the training task during an immediate post-training assessment when HR was not present. However, individuals were not able to reduce their HR responses to tasks during the short delay and long delay post-training sessions and they were unable to transfer HR reduction skills to a novel task (i.e., the speech task). In general, blood pressure responses to the tasks during the post-training sessions were equivalent to pre-training blood pressure levels. Individuals demonstrated consistent performance levels during the videogame and hand grip tasks, and increasing performance levels during the mental arithmetic task. Additional research is needed to examine whether providing motivation (e.g., monetary rewards) during post-training sessions or teaching specific HR reducing skills (e.g., diaphragmatic breathing) might enhance the transfer of HR feedback training and the reduction of HR responses to any number of tasks. 


\section{Acknowledgements}

First, and foremost, I gratefully acknowledge the guidance, assistance, and friendship of my "senior colleague" Kevin Larkin. I have learned from Kevin, not only how to excel as a researcher, clinician, and teacher, but also the value of balancing "work life" and "home life," which he appears to do so expertly. Without Kevin's guidance and assistance this document, and all of the milestones that have preceded its development, may have never materialized. Second, I extend my most sincere gratitude to each of my committee members, Marty, Barry, Dave, and Jeannie, whose comments and suggestions were integral in the development of this project. Each of these individuals has played an essential role in shaping my skills, but even more importantly each serves as a role model for my professional career. I am quite fortunate to have had their assistance throughout my career. Third, I like to thank Brandie Taylor, Nicole Siegwarth, and Damien White for their assistance and hard work in completing this project. In particular, I'd like to thank Brandie for her flexibility and willingness to help whenever I needed an extra hand. Finally, I thank my family, Mary, Alex, and Zach, who have endured having their husband/father away from home or tied to a computer for long hours and often not providing them with the attention or assistance that they deserve. My family's unconditional love has always served as my motivation to continue to reach for my goals and their support made the completion of this project possible. 


\section{Table of Contents}

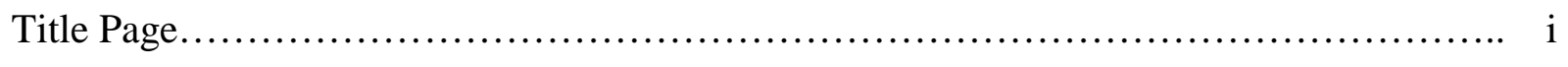

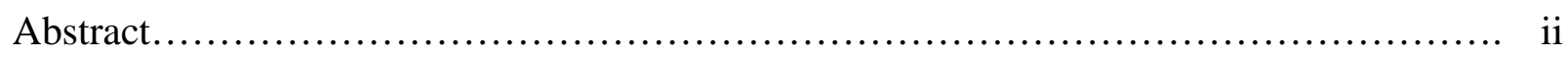

Acknowledgements......................................................... iii

List of Tables.........................................................

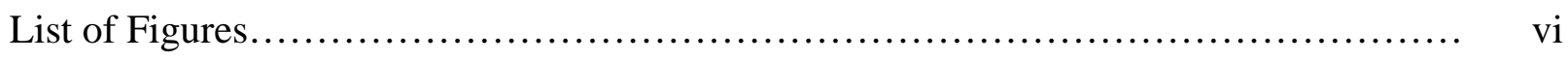

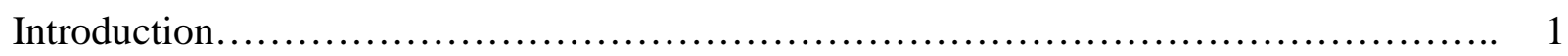

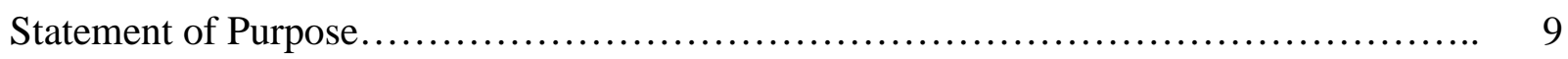

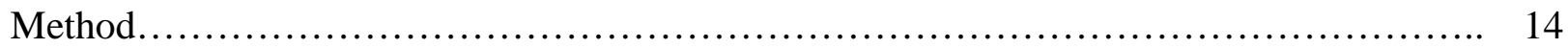

Participants........................................................ 14

Tasks............................................................. 16

Measures............................................................ 17

Procedure.......................................................... 19

Results................................................................... 25

Discussion........................................................... 45

References........................................................... 59

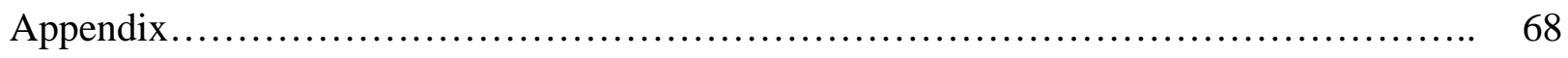

Curriculum Vita........................................................ 115 
List of Tables

Table 1. Demographic information of individuals participating in the pre-training

assessment............................................................. 81 


\section{List of Figures}

Figure 1. Pre-training heart rate responses to videogame, mental arithmetic, hand grip, and speech tasks for all participants completing the pre-training assessment. Change scores were calculated by subtracting lowest baseline heart rate from average heart rate observed during the task presentation. $\mathrm{VG}=$ videogame; $\mathrm{MA}=$ mental arithmetic; $\mathrm{HG}=$ hand grip; $\mathrm{SP}=$ speech; $\mathrm{HR}=$ heart rate.

Figures 2-3. Lowest baseline heart rate levels recorded during each phase of the study. Pre-train = pre-training; VG-\# = videogame training session \#; VG-Post = videogame immediate post-training session; MA-\# = mental arithmetic training session \#; MA-Post $=$ mental arithmetic immediate post-training session; $\mathrm{HG}-\#=$ hand grip training session \#; HG-Post $=$ hand grip immediate post-training session; $\mathrm{P} 1=$ short delay post-training session; $\mathrm{P} 2$ = long delay post-training session; $\mathrm{HR}=$ heart rate..

Figures 4 -9. Heart responses to videogame, mental arithmetic, hand grip, and speech tasks across pre-training, training, and post-training sessions. Responses to the videogame are displayed with blue diamonds, responses to the mental arithmetic task are displayed with red squares, responses to the hand grip task are displayed with green triangles, and responses to the speech task are displayed with black circles. The first session is the pre-training session. Training sessions range from 1 to 3 and are followed by an immediate post-training session. The final two phases of the graph represent short delay and long delay post training sessions. Heart responses were calculated by subtracting the lowest baseline heart rate during the pre-training session from the average heart rate observed during the presentation of the tasks. $\mathrm{PT}=$ pre-training; $\mathrm{VG}=$ 
videogame; $\mathrm{MA}=$ mental arithmetic $\mathrm{HG}=$ hand grip $\mathrm{SP}=$ speech $; \mathrm{I}=$ immediate posttraining session; $\mathrm{SD}=$ short delay post-training; $\mathrm{LD}=$ long delay post-training.

Figures 10-15. Systolic blood pressure responses to videogame, mental arithmetic, hand grip and speech tasks during pre-training and post-training periods. Responses to the videogame are displayed with blue diamonds, responses to the mental arithmetic task are displayed with red squares, responses to the hand grip task are displayed with green triangles, and responses to the speech task are displayed with black circles. $\mathrm{VG}=$ videogame; $\mathrm{MA}=$ mental arithmetic $\mathrm{HG}=$ hand grip; $\mathrm{SP}=$ speech .

Figures 16-21. Diastolic blood pressure responses to videogame, mental arithmetic, hand grip and speech tasks during pre-training and post-training periods. Responses to the videogame are displayed with blue diamonds, responses to the mental arithmetic task are displayed with red squares, responses to the hand grip task are displayed with green triangles, and responses to the speech task are displayed with black circles. $\mathrm{VG}=$ videogame; $\mathrm{MA}=$ mental arithmetic; $\mathrm{HG}=$ hand grip; $\mathrm{SP}=$ speech.

Figures 22-27. Performance scores during the videogame, mental arithmetic, and hand grip tasks across pre-training, training, and post-training sessions. Responses to the videogame are displayed with blue diamonds, responses to the mental arithmetic task are displayed with red squares, responses to the hand grip task are displayed with green triangles, and responses to the speech task are displayed with black circles. The first session is the pre-training session. Training sessions range from 1 to 3 and are followed by an immediate post-training session. The final two phases of the graph represent short delay and long delay post training sessions. $\mathrm{PT}=$ pre-training; $\mathrm{VG}=$ videogame; $\mathrm{MA}=$ 
mental arithmetic; $\mathrm{HG}=$ hand grip; $\mathrm{I}=$ immediate post-training session; $\mathrm{SD}=$ short delay post-training; $\mathrm{LD}=$ long delay post-training.

Figures 28-33. SUDs ratings during the videogame, mental arithmetic, hand grip, and speech tasks across pre-training, training, and post-training sessions. Responses to the videogame are displayed with blue diamonds, responses to the mental arithmetic task are displayed with red squares, responses to the hand grip task are displayed with green triangles, and responses to the speech task are displayed with black circles. The first session is the pre-training session. Training sessions range from 1 to 3 and are followed by an immediate post-training session. The final two phases of the graph represent short delay and long delay post training sessions. $\mathrm{PT}=$ pre-training; $\mathrm{VG}=$ videogame; $\mathrm{MA}=$ mental arithmetic; $\mathrm{HG}=$ hand grip; $\mathrm{SP}=$ speech; $\mathrm{I}=$ immediate post-training session; $\mathrm{SD}$ $=$ short delay post-training; $\mathrm{LD}=$ long delay post-training. 


\section{Introduction}

Cardiovascular disease (e.g., hypertension, coronary heart disease, strokes) accounts for $41 \%$ of deaths per year, and since the 1930 s has been the primary cause of death in the United States (American Heart Association, 1997). Moreover, epidemiological evidence suggests that one in five individuals have some form of cardiovascular disease (American Heart Association). The two most common forms of cardiovascular disease, coronary heart disease and hypertension, account for $61 \%$ of cardiovascular diseases (American Heart Association).

Coronary heart disease develops over time as arteries harden through the process of atherosclerosis, which is the build-up of plaque on the interior lining of the arteries. Increased plaque levels may lead to arterial blockage and eventually impede the flow of blood to the heart (i.e., a heart attack) or to the brain (i.e., a stroke). Plaque tends to form in places where the endothelial lining of the artery is damaged, which often occurs at sites of increased blood turbulence (e.g., branches of arteries proximal to the heart). Elevated blood pressure promotes endothelial damage and is an important factor in the development of cardiovascular disease (Smith \& Leon, 1992). The development of hypertension, which is chronically elevated blood pressure (systolic blood pressure $(\mathrm{SBP})>140 \mathrm{~mm} \mathrm{Hg}$ and/or diastolic blood pressure (DBP) > $90 \mathrm{~mm} \mathrm{Hg}$ ) has been shown to be an important risk factor in the development of coronary heart disease. In addition to hypertension, other risk factors have been related to the development of coronary heart disease. These risk factors include both uncontrollable factors, such as heredity and age, and many other modifiable risk factors such as smoking, serum cholesterol level, obesity, and physical inactivity. However, the three leading risk factors (i.e., hypertension, serum cholesterol levels, and smoking) only account for $50 \%$ of the variance in the prediction of coronary heart disease (Jenkins, 1988). 
Researchers have examined a variety of behavioral factors that may explain additional variance in the prediction of coronary heart disease and other cardiovascular diseases. For example, hostility (e.g., Barefoot, Larsen, Von der Leith, \& Schroll, 1995), lack of social support, (e.g., Orth-Gomer, 1994; Ruberman, Weinblatt, Goldberg, \& Chaudhary, 1984), and depression (e.g., Booth-Kewley \& Friedman, 1987; Frasure-Smith, Lesperance, \& Talajic, 1993) are all believed to be related to the development of cardiovascular disease. Additionally, physiological responses to stress have been widely studied as behavioral risk factors for cardiovascular disease (Allan \& Scheidt, 1996).

Researchers have long observed that individuals exhibit different physiological reactions to stress (Lacey, Bateman, \& Vanlen, 1953; Moos \& Engel, 1962). These observations contributed to the concept of individual response specificity, which posits that a single person will demonstrate a consistent pattern of physiological responses to a variety of stimuli, but the pattern of physiological responses may differ between persons (Andreassi, 1995; Lacey, Bateman, \& Vanlen). Applying the concept of individual response specificity to the cardiovascular system, researchers have shown that certain individuals demonstrate exaggerated cardiovascular responses to engaging, aversive, or challenging stimuli (i.e., stressors) compared to others persons presented with the same stimuli (Manuck, 1994).

\section{The Cardiovascular Reactivity Hypothesis}

Malmo (1950) suggested that physiological reactivity to stress may be related to disease processes. For example, when individuals encounter an engaging, aversive, or challenging situation, the sympathetic nervous system is activated. Sympathetic nervous system activation results in heart rate (HR) and blood pressure increases to prepare muscle tissue for a "fight or flight" response. Although an adaptive response, this increased cardiovascular response may 
place additional strain on the vasculature leading to the development of atherosclerotic lesions, plaque build-up, and eventually hypertension or coronary artery disease. Combining the theory of individual response specificity and the relation of physiological reactivity to disease, researchers (Krantz \& Manuck, 1984; Manuck, Kasprowicz, Monroe, Larkin, \& Kaplan, 1989) have developed the cardiovascular reactivity hypothesis. Specifically, the cardiovascular reactivity hypothesis posits that individuals demonstrating exaggerated cardiovascular responses to stressful stimuli have a greater risk of developing cardiovascular disease compared to individuals with lower levels of responding to the same stimuli (Manuck et al., 1989; Manuck, 1994).

Animal, case-control, and prospective studies have provided evidence to support the cardiovascular reactivity hypothesis. Manuck, Kaplan, and Clarkson (1983) examined cardiovascular responses in 22 cynomolgus monkeys fed an atherogenic diet (i.e., high in fat) for 22 months. Monkeys exhibiting higher heart rate reactions to a stressor (i.e., threat of capture) several weeks before necropsy, also demonstrated greater levels of coronary atherosclerosis compared to those with lower heart rate reactivity to the stressor. These findings were later replicated in a sample of female cynomolgus monkeys (Manuck, Kaplan, Adams, \& Clarkson, 1989).

Case-control studies have also been used to explore the relation between cardiovascular reactivity to stress and cardiovascular disease (e.g., Corse, Manuck, Cantwell, Giordani, \& Matthews, 1982; Hastrup, Light, \& Obrist, 1982; Hollenberg, Williams, \& Adams, 1981). For example, Corse et al. (1982) examined adult males with and without a history of coronary heart disease, but matched on a variety of variables including, age, race, and family history of cardiovascular disease. Corse et al. found that those demonstrating exaggerated DBP responses 
to stress were more likely to be diagnosed with coronary heart disease. A meta-analysis of casecontrol studies found that, compared to normotensive individuals, hypertensive individuals demonstrated exaggerated cardiovascular responses to a variety of stressors (Fredrickson \& Matthews, 1990).

Prospective evidence has generally supported the cardiovascular reactivity hypothesis. Prospective studies typically examine HR and BP responding to a laboratory stressor (e.g., cold pressor, mental arithmetic challenge) and then examine the development of cardiovascular disease (e.g., hypertension, coronary heart disease, atherosclerotic build-up) several years later. Prospective studies (e.g., Barnett, Hines, Schirger, \& Gage, 1963; Barnett, Spence, Manuck, \& Jennings, 1997; Davidoff et al. 1982; Keys et al., 1971; Matthews et al., 1998; Menkes et al., 1989) have consistently demonstrated a positive relation between exaggerated responses to laboratory stressors and the development of a disorder of the cardiovascular system among Caucasian men. However, the evidence regarding women and persons of different racial composition is less clear. More specifically, individuals who demonstrate higher HR and/or BP reactions to laboratory stressors, particularly the cold pressor task and exercise tasks, are also more likely to develop cardiovascular disease than their non-reactive counterparts. $\underline{\text { Reducing Cardiovascular Reactivity }}$

Given the potential negative consequences of exaggerated cardiovascular responses to stress, it would seem desirable to develop and test methods to control and moderate HR and/or physiological responses to challenging, engaging, or aversive stimuli. Moderating cardiovascular reactivity, like eliminating smoking, increasing exercise, or reducing sodium and fat intake, may reduce one's risk for developing coronary heart disease and hypertension. Researchers have examined several methods aimed at reducing one's physiological reaction to stress, including 
cognitive (Grimm \& Kanfer, 1976; Houston \& Holmes, 1976), behavioral (Ewart, Burnett, \& Taylor, 1983; Kirsch \& Henry, 1979), and physiological interventions (Goleman \& Schwartz, 1976; Lehrer, 1978; Sawada \& Steptoe, 1988). Studies examining the efficacy of methods to reduce cardiovascular response to stress typically involve random assignment of participants to a control group or a treatment group. The procedure generally includes three phases: pre-training, training, and post-training. In the pre-training phase, HR or BP measures are assessed while the participant is at rest and during exposure to a standardized stressor (e.g., shock, cold pressor, mental arithmetic). Change scores from the resting state to the presentation of the stressor provide an initial estimate of cardiovascular reactivity. Next, during the training phase, individuals in the treatment group receive instruction in the method designed to reduce their cardiovascular response (e.g., cognitive intervention, relaxation, biofeedback) while control participants receive no training. In some investigations, participants have received training while at rest (Blanchard \& Young, 1972; Hatch, 1980; Manuck, Levenson, Hinrichsen, \& Gryll, 1975), whereas in other studies, training was conducted while participants engaged in a physical or mental task (Larkin, Manuck, \& Kasprowicz, 1990; Perski \& Engle, 1980; Sirota, Schwartz, \& Shapiro, 1974). Finally, in the post-training phase of these studies, participants in both groups are re-exposed to the stressor and a second estimate of cardiovascular responsivity is calculated. Researchers can then compare change in cardiovascular response magnitude of those in the treatment group with control participants to determine whether training affected cardiovascular responding to stress.

Research examining the efficacy of the various non-pharmacological methods to decrease HR responses to stress has resulted in discrepant findings. Some studies demonstrated that nonpharmacological methods effectively decrease HR responses (Goleman \& Schwartz, 1976; 
Grimm \& Kanfer, 1976; Kirsch \& Henry, 1979; Larkin, Manuck, \& Kasprowicz, 1989) and others have failed to support this contention (e.g., Ahles, Blanchard, \& Leventhal, 1983; Falkowski \& Steptoe, 1981; Green, Webster, Beiman, Rosmarin, \& Holliday, 1981; Jorgenson, Houston, \& Zurawski, 1981; Sharpley, 1989). Although it is beyond the scope of this paper to review the entire body of literature pertaining to the effectiveness of these methods for reducing cardiovascular reactivity to stress, two findings are important to highlight. First, HR feedback has been shown to reduce HR reactivity to a stressor more consistently than other interventions (e.g., cognitive restructuring, relaxation therapy). Second, interventions that involved training during the presentation of a stressor (e.g., Ainslie \& Engel, 1974; Larkin et al., 1989; Larkin, Zayfert, Veltum, \& Abel, 1992; Sirota et al., 1974), rather than during periods of rest (e.g., Bennett, Holmes, \& Frost, 1978; Blanchard \& Young, 1972; Carroll \& Evans, 1981; Hatch, 1980; Manuck, et al., 1975), have been more effective at reducing HR reactivity to stress. Although these findings have been consistently observed, the role of training during stressor presentation has rarely been examined empirically. Bentham and Glaros (1982) conducted the only published study to date directly comparing training during a stressor and at rest. Using pulse transit time feedback, the researchers found that only those participants trained during the stressor demonstrated reductions in cardiovascular reactivity to stress (increased pulse transit time). Participants in a no treatment group and those trained during rest showed no change in cardiovascular reactivity to stress. In sum, studies examining the effects of HR feedback training upon cardiovascular reactivity to stress by employing animals (Ainslie \& Engel, 1974; Engel \& Chism, 1967; Engel \& Gottlieb, 1970; Engel \& Hansen, 1966) and humans exposed to shock (Sirota et al., 1974), physical exercise (Goldstein, Ross, \& Brady, 1977; Perski \& Engel, 1980), and videogame challenges (Larkin et al., 1989; Larkin et al., 1990; Larkin, Zayfert, Veltum, \& 
Abel, 1992) have demonstrated that HR biofeedback can reliably reduce exaggerated HR responses to stressors when participants are trained to reduce their HR during the presentation of a stressor. Considering these findings, it is evident that the most promising method for obtaining reductions in behaviorally-elicited cardiovascular responsivity is HR feedback training during the presentation of a stressor.

\section{Transfer of HR Feedback Training}

Although studies have consistently demonstrated that individuals can effectively reduce HR when trained during the presentation of the stressor (Ainslie \& Engel, 1974; Engel \& Chism, 1967; Engel \& Gottlieb, 1970; Engel \& Hansen, 1966; Goldstein, Ross, \& Brady, 1977; Larkin et al., 1989; Larkin et al., 1990; Larkin, Zayfert, Veltum, \& Abel, 1992; Perski \& Engel, 1980; Sirota et al., 1974), few studies have examined whether an individual can utilize the HR reduction skills learned during one stressor and apply those skills during the presentation of a different stressor. Among all studies in this literature, only three studies (Goodie \& Larkin, 1999; Larkin, Zayfert, Abel, \& Veltum, 1992; Sharpley, 1994,) have examined the transfer of HR feedback training; that is, these studies examined whether skills learned during HR feedback training could be used to reduce HR during the presentation of another stressor not used during training.

Larkin, Zayfert, Abel, and Veltum (1992) investigated whether HR feedback training would transfer (a) to sessions conducted one week later, and (b) to a mental arithmetic task that was not used during feedback training. HR reactivity to the videogame and mental arithmetic task was assessed in 8 experimental and 8 control participants. Participants in the experimental group were trained using five HR feedback-training trials, whereas participants in the control group were simply instructed to lower HR without feedback. Following training, participants 
were again exposed to the videogame and mental arithmetic tasks and instructed to use "whatever skills they had learned to lower their HR." One week later, participants returned and were provided with the same instructions they received during the post-training session. Results showed that those participants trained to reduce their HR using feedback, compared to control group participants, demonstrated significantly less HR reactivity to both the videogame and mental arithmetic tasks during the post-training and one-week follow-up sessions. These findings suggested that HR reductions obtained with biofeedback training transferred to tasks not used in training as well as across time.

Goodie and Larkin (1999) also examined whether training during a videogame stressor transferred to a mental arithmetic challenge. After pre-training cardiovascular reactivity to both a videogame and mental arithmetic challenge was assessed, half of the participants were provided with HR feedback using the videogame challenge during 5 training trials (FB+ group). During a post-training assessment of cardiovascular responses to the videogame and mental arithmetic challenge, participants who received HR feedback training were asked to "use the skills that they had learned to keep their HR as low as possible during both tasks." Although, HR feedback participants demonstrated significantly lower HR responding during the post-training videogame, there were no significant differences between the two groups during the mental arithmetic challenge. In contrast to Larkin et al. (1992), these results suggested that the skills obtained during HR feedback training during the videogame failed to transfer to the mental arithmetic challenge. A noteworthy difference between the Goodie and Larkin and Larkin et al. studies is that the latter reported that the largest reduction of HR reaction to the mental arithmetic task for $\mathrm{FB}+$ participants during the follow-up session 1-week later. Because Goodie and Larkin did not include a follow-up session, it is unknown whether the participants from this study would have 
demonstrated lowered HR responses to the mental arithmetic at a later time. Otherwise the protocols were quite similar.

Sharpley (1994) provided evidence that individuals who learned to reduce HR with biofeedback were able to transfer what they learned to non-laboratory situations. Sharpley (1994) demonstrated that individuals who received biofeedback, imagery, breathing training, and education about cardiovascular disease reduced HR reactivity to mental stress more than control participants. Treatment group participants returned once a week for five weeks to receive HR feedback training during a laboratory mental arithmetic stressor. HR reactivity was assessed for the mental arithmetic task three times prior to training and three times following training. Work HR reactivity (i.e., HR changes recorded during a person's daily activities while behavioral observations were made by an observer) was assessed for two hours two times prior to HR feedback training and two times following training. The treatment group showed significantly lower HR responses to the mental arithmetic task during all three post-training sessions and demonstrated smaller HR reactivity during work than the control group. According to the authors, $95 \%$ of the participants who received biofeedback training reported that they continued to use the training 2.5 years later. However, because Sharpley trained individuals to use a variety of skills to reduce $\mathrm{HR}$, it is unclear how HR feedback training uniquely contributed to the reduced HR response to stress at work.

\section{Statement of Purpose}

Although researchers have demonstrated that individuals can learn to reduce HR responses to mental stressors using HR feedback, it is unclear how well individuals can apply these skills in the presence of stressors not used during training. The results from Larkin et al. (1992), Goodie and Larkin (1999), and Sharpley (1994) provide mixed evidence about the 
efficacy of HR feedback training in the presence of stressors not used during training. One explanation for the inconsistent findings regarding the transfer HR feedback training during nontraining tasks is that individuals were unable to apply skills used during the training task to other tasks due to the incongruence between the stimulus and response features of the training task (e.g., videogame) and "testing" task (e.g., mental arithmetic). It is unlikely that a single stressor captures the complex topography of the multitude of laboratory stressors or the multiple daily stressors experienced by most individuals. Each of these researchers used a single stressor to provide HR feedback training. Stressors vary widely in their topography and involve different stimulus features and require varying responses to effectively manage the demands of the task, which may limit the transfer of HR reduction skills between tasks. For example, some challenges, such as a videogame, may present performance demands on the individual, involving visual stimuli (i.e., watching the game) and motor responses (i.e., moving the joystick), whereas a mental arithmetic task involves auditory stimuli (i.e., verbal presentation of numbers), cognitive skills (i.e., mathematical computations), and verbal responses (i.e., stating the answer). Physical challenges, such as a hand grip task, involve muscular endurance and the maintenance of a physical response (i.e., gripping the hand dynamometer). Therefore, to effectively train individuals to use skills gained from HR feedback training it may be necessary to provide HR feedback training using a variety of stressors with varying stimulus and response features.

Examining HR feedback training using multiple training stressors presented during multiple training sessions would require many hours of training for each participant. If a traditional between-subjects study design was used, a project of this type may be impractical due to the amount of time spent training each participant and the cost in paying participants for their time and effort. Weems (1998) suggested that single-subject research designs may be helpful for 
examining the effects of HR feedback on physiological functioning. In a study with a singlesubject design, the individual serves as the unit of analysis and the variability of performance within each of the experimental conditions is examined (Lejuez, Zvolensky, \& Eifert, 1999). For example, a single-subject design allows researchers to examine how an intervention, such as HR feedback training, affects physiological responding to stimuli for each individual.

There are various types of single-subject design studies, including reversal-replication (e.g., A-B-A-B) and multiple baseline designs. These designs allow researchers to rule-out secondary explanations (e.g., habituation) for the observed treatment effects. During the first phase of reversal-replication design, the target behavior response (e.g., HR during a task) is measured prior to an intervention (i.e., A). In the second phase, the target behavior is measured during the presentation of the intervention (i.e., B). In the third phase, the intervention is withdrawn and the target response is expected to return to the level observed during the initial assessment period. Finally, the intervention is then reintroduced (i.e., B), and responding is expected to occur at the same level as observed during the first presentation of the intervention. However, a reversal-replication design is not conducive to testing skill development because once a skill is learned during an intervention, the skill does not become unlearned when the intervention is withdrawn. Consequently, a reversal-replication design is not appropriate for examining transfer of HR feedback training.

There are various types of multiple baseline designs. In one multiple baseline design, the baseline levels of the target response is measured across several tasks as an intervention is introduced sequentially for each task. For example, the target behavior response (e.g., HR response) to three stimuli (e.g., videogame, mental arithmetic, and hand grip task) is assessed during a pre-intervention phase. Then an intervention (e.g., HR feedback training) is introduced 
in the presence of one stimulus (e.g., videogame task) while the target behavior is measured during the presentation of all of the stimuli. The intervention is then presented during the presentation of a second stimulus (e.g., mental arithmetic), and the target response is again measured during the presentation of each stimulus. Finally, the intervention is presented during the presentation of the third stimulus. In order to obtain the desired effect, training during each stimulus should not transfer to the other tasks before training is provided with them. In the case of HR reduction to behavioral tasks, it is possible that transfer could result to other tasks without training, thus preventing the observed sequential training effect seen in multiple baseline designs. A second version of the multiple baseline design, which involves obtaining multiple baseline levels across subjects rather than tasks, helps to address some of the limitations of the betweentask multiple baseline design. In the between-subject multiple baseline design, the intervention for each participant is introduced sequentially. For example, after a pre-training assessment of the target response, the first individual would receive the intervention. A second individual would have the target response measured during two pre-training sessions, and have the intervention introduced during the third session. Similarly, for a third individual, the target response would be measured for three pre-training sessions, and the intervention would be introduced during the fourth session. A combination of both of the aforementioned multiple baseline designs is necessary to distinguish habituation from the actual learning of a skill that is applied during multiple tasks (e.g., HR response reduction).

The purpose of the current study was to examine the transfer of HR feedback training to reduce HR reactions to stress using a multiple baseline single subject design. Unlike previous studies, this study trained individuals to use HR feedback during the presentation of multiple stressors, rather than employing a single stressor during training. Specifically, individuals were 
provided with HR feedback training during three different tasks, comprised of a variety of stimulus-response characteristics: a videogame, mental arithmetic, and a hand grip challenge. Participants received HR feedback training during each challenge sequentially until they reduced their HR in the presence of that stressor, or after 3 training sessions. After individuals reduced their HR during each of the three training challenges, participants were asked to reduce their HR without HR feedback in the presence of the three training stressors (i.e., videogame, mental arithmetic, and hand grip) and a stressor not used during training (i.e., speech task). Approximately, one week later, participants were asked to return to the laboratory and again attempt to reduce their HR responses during the presentation of the three training tasks (i.e., videogame, mental arithmetic, hand grip) and one non-training task (i.e., speech task).

\section{Hypotheses}

1. Based on previous HR feedback training studies (e.g., Goodie \& Larkin, 1999; Larkin et al., 1989; Larkin et al., 1990; Larkin, Zayfert, Veltum, \& Abel, 1992) that demonstrated persons can reduce HR responses to tasks during HR reduction training, it was expected that participants would reduce HR responses to each task (i.e., videogame, mental arithmetic, hand grip) when HR feedback was provided during the presentation of that task.

2. The HR feedback training studies also demonstrated that individuals could reduce HR responding without HR feedback after receiving HR feedback training. Therefore, it was expected that following training on all 3 tasks, individuals would lower their HR responses, without the aid of HR feedback, during the presentation of each stressor that was used during training.

3. Following HR feedback training during the videogame, mental arithmetic, and hand grip challenges, individuals would demonstrate a lower HR response during the speech task 
compared to pre-training HR responses. Following the individual training sessions for each task, it was expected that individuals would demonstrate the lowest HR responding during the task(s) that were previously used during training. That is, the training tasks were presented as probe tasks after each training session, whereas the speech task was presented as a probe task during the post-training sessions only.

4. After a one-week follow-up period, individuals would demonstrate reduced HR responses during the presentation of all of the challenges compared to pre-training HR responses.

Method

\section{$\underline{\text { Participants }}$}

Fourteen individuals were recruited from undergraduate psychology courses at West Virginia University. Individuals taking cardiovascular-reactive drugs (e.g., decongestants, asthma medication, or beta-blockers) or who had a history of cardiovascular disease were excluded from the study. Participants were asked not to consume alcohol for 24 hours prior to coming to each laboratory session. Participants were also asked to refrain from smoking for 3 hours and avoid eating or exercising for 1 hour prior to attending each laboratory session. Table 1 displays the demographic characteristics of the participants who completed the pretraining session. Two individuals (participants 1 and 3) who attended the pre-training sessions were not invited to participate because EKG signals could not be reliably measured (i.e., electrical interference). Two participants (i.e., participants 6 and 9), discontinued the study after completing the pre-training sessions. Of the remaining subjects, the six individuals (i.e., participants $2,4,5,10,11$, and 13) demonstrating the highest average HR responses to the tasks were selected to complete the training and post-training sessions. Figure 3 displays the HR changes from baseline observed during the pre-training session. Although the speech task was 
done only once at pre-treatment, participants completed from 3 to 8 trials at pre-treatment for the remaining three tasks.

\section{$\underline{\text { Design }}$}

A single subject, multiple baseline study design was used to examine the transfer of $\mathrm{HR}$ feedback training. First, a pre-training session was used to examine baseline HR responses to four laboratory stressors (i.e., a videogame, mental arithmetic, hand grip, and speech tasks). The videogame, mental arithmetic, and hand grip tasks each were presented until the individual demonstrated consistent HR responses; that is, the individual's HR responses were within 5 bpm of each other across 3 consecutive trials. Following the pre-training session, several training sessions for the videogame, mental arithmetic, and handgrip tasks were conducted. During each training session, individuals were asked to reduce their HR while HR feedback was presented during completion of one of the three training tasks. After the individuals reduced their HR to the specified criteria or after three training sessions for a task, which ever occurred first, the participant moved on to training with the next task. A limit of three training sessions was implemented because previous research (i.e., Goodie \& Larkin, 1999; Larkin et al., 1989; Larkin et al., 1990; Larkin, Zayfert, Veltum, \& Abel, 1992) suggested that individuals typically learned to reduce their HR response to the videogame task during the course of a single session. Therefore, it was presumed that if individuals did not learn to lower their HR response to the tasks after three 2-hour sessions, that it was unlikely that they would successfully lower their HR response, regardless of the number of training trials. Following training with each task, the videogame, mental arithmetic, and hand grip tasks were presented without HR feedback. One to two days following the final training session, participants were asked to attend a post-training session (i.e., short delay). During the short delay post-training assessment participants were 
asked to reduce their HR, without HR feedback, during the presentation of the videogame, mental arithmetic, hand grip, and speech challenges. Participants were then asked to return approximately one week later for a second post-training session (i.e., long delay). During the long delay session, participants were again asked to reduce their HR during the presentation of the videogame, mental arithmetic, hand grip, and speech tasks.

\section{$\underline{\text { Training Tasks }}$}

Videogame. The Sno-Cat videogame required subjects to steer an image of a motorized snowmobile up a snow-covered mountain, while avoiding trees that randomly appeared in its path. Each presentation of the videogame lasted 2-minutes. The subjects could control the horizontal movements of the Sno-Cat as well as slow it down using the firebutton as a brake. However, the overall speed of the Sno-Cat could not be controlled by the subject. The Sno-Cat videogame was presented using a Commodore 64 computer using a Commodore disk drive (Model 1524), and a Commodore color monitor (Model 1802). Subjects controlled the

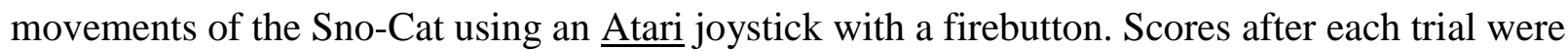
recorded by the researcher. The Sno-Cat program provided HR feedback by changing the background colors of the screen. This videogame task was successfully used to teach HR feedback in previous studies (Goodie \& Larkin, 1999; Larkin et al., 1989; Larkin et al., 1990; Larkin, Zayfert, Veltum, \& Abel, 1992)

Mental Arithmetic. The mental arithmetic task required subjects to count backwards by 17s from a four-digit number that was verbally presented to them using a cassette player. The task lasted 2 minutes and a new four-digit number was presented at the beginning of each minute of the task. Participants were asked to work "as quickly and accurately as possible." HR feedback was provided using changing background colors on a computer screen. Previous 
studies (Goodie \& Larkin, 1999; Larkin, Zayfert, Abel, \& Veltum, 1992) used this mental arithmetic task to examine the transfer of skills learned during HR feedback training.

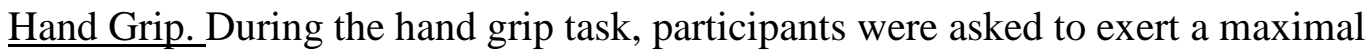
voluntary contraction using a hand dynamometer, and then sustain a force of 30 percent of maximum voluntary contraction for two minutes. The hand grip challenge served as a physical stressor for participants. The hand grip challenge has been used in laboratory investigations of cardiovascular responding, and shown to elicit significant heart rate and blood pressure responses (Faultisch et al., 1986). Again, changing colors on the computer monitor served as feedback to participants about their HR levels. The hand grip task has not been used in previous HR feedback training studies.

$\underline{\text { Task to Test Transfer of Training }}$

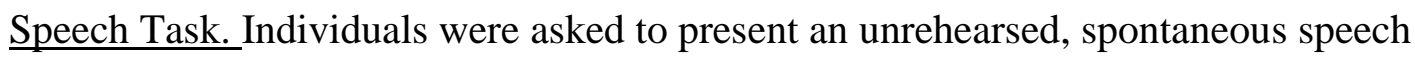
about a controversial topic for a 2 -min period. The topic of gun control was used during the pretraining session, the topic of the right to die was used during the first post-training session, and the topic of abortion was used during the final post-training session. Participants were told that their responses would be recorded and that the clarity of their argument would be evaluated. Presentations of a prepared speech have been employed in past research (Matthews, Manuck, \& Saab, 1986; Turner, Carroll, Dean, \& Harris, 1987; Turner, Girdler, Sherwood, \& Light, 1990), and have been shown to elicit significant pressor and heart rate responses.

\section{$\underline{\text { Measures }}$}

\section{$\underline{\text { Cardiovascular Assessment }}$}

$\underline{\text { Heart Rate at Pre-Training and Post-Training. The Minnesota Impedance Cardiograph }}$ (Model \#304B) was used to obtain measures of HR when the individual was presented with the 
tasks during the pre-training and post-training sessions, and when the individual was at rest. Three surface electrodes were used to detect the EKG signal. The first electrode was placed on the right side below the collar bone, the second electrode was placed on the contra-lateral side between two ribs, and the third electrode (i.e., the ground) was placed on the right side of the individual near the abdomen. The EKG waveforms were transmitted to an IBM compatible computer for display, ensemble scoring, and recording purposes.

Heart Rate During Training. A finger photoplethysmogram (Lafayette, Model \#76624) provided a continuous measure of HR during periods when HR feedback was provided (i.e., during the training sessions). The device was placed on the middle finger of the subject's nondominant hand. Pulse waves were translated into beats per minute by a Lafayette Heart Rate Monitor (Model \#77067). To provide HR feedback, the HR monitor was connected to a Schmitt Trigger (Lafayette Model \#76729) that was attached to the Commodore 64 computer through the orange and black leads of an Atari joystick plugged into control port \#2 of the computer. For HR feedback training, HR values were entered into the Heart Rate Monitor. When the participant's HR exceeded the criterion value entered into the Heart Rate Monitor, a signal was sent to the Schmitt Trigger, which closed the relay. When the individual's HR was below the criterion level, the Schmitt Trigger relay remained open. The heart rate feedback program checked the status of the relay approximately every two seconds and provided appropriate feedback to the participant. The background color of the screen turned red to indicate increased HR, blue to indicate decreased HR, and white indicating no change in HR.

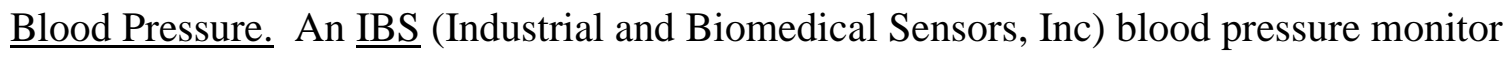
was used to assess systolic blood pressure and diastolic blood pressure. During times when feedback was not presented (e.g., during pre- and post-training sessions), the blood pressure cuff 
was fastened around the participant's non-dominant arm and inflated every two minutes to provide blood pressure values. During the training periods, when HR feedback was provided, no blood pressure readings were taken because such readings would interfere with the HR signal (i.e., the inflation of the cuff would limit blood flow to the finger being used to assess HR). A microphone inside of the cuff detected Korotkoff sounds from the brachial artery and provided measures of BP, which were digitally displayed and recorded by the experimenter.

\section{$\underline{\text { Self-Reported Arousal }}$}

To obtain a self-report measure of arousal, participants were asked to rate their Subjective Units of Discomfort (SUDs) throughout the training sessions as well as pre- and post-training assessment sessions. Previous studies examining HR feedback training have not typically examined arousal ratings associated with HR reduction after training. The SUDs ratings were used to examine whether reductions in HR responses were associated with reductions in selfreported arousal. The scale ranged from 0 , the most relaxed individuals could imagine, to 100 , the highest level of stress that individuals could imagine. Individuals recorded their SUDs ratings on a form provided to them (Appendix A).

\section{$\underline{\text { Procedure }}$}

The procedure for the study included a pre-training session, a maximum of three training sessions per training task, a short-delay post-training assessment (i.e., 1-2 days following the final training session), and a long-delay post-training assessment (i.e., 1-2 weeks following the final training session). A flow chart for the procedures of the study are presented in Appendix B.

Pre-Training Assessment Session. Individuals volunteering for the study, and meeting acceptance criteria, were asked to come to the psychophysiology laboratory in Oglebay Hall. Informed consent (Appendix C) was obtained from all participants and a general summary of the 
study was provided. A demographics form (Appendix D) was then administered and compliance with pre-session instructions was assessed. Participants were asked to sit in a comfortable chair while the finger photoplethysmogram, three EKG electrodes, and blood pressure cuff were attached. Following the attachment of the physiological equipment, a 10-min habituation period commenced.

The presentation order of the four pre-training tasks (i.e., the videogame, mental arithmetic, hand grip, and speech tasks) was the same for all participants. The same task order was used for all participants because previous studies (e.g., Goodie \& Larkin, 1999, Larkin et al., 1989; Larkin et al., 1990; Larkin, Zayfert, Veltum, \& Abel, 1992) demonstrated that individuals can successfully reduce HR using HR feedback during the videogame and mental arithmetic challenges, however, there are no studies that have examined HR training during the hand grip task. Therefore, the videogame and mental arithmetic tasks were presented first to maximize the likelihood that individuals learned to reduce their HR successfully. No HR feedback was provided during the pre-training session and SUDs ratings were obtained at the end of each rest period and task. A 4-minute baseline followed by instructions for the upcoming task preceded each challenge.

During the first task, the Sno-Cat videogame, subjects were asked to play multiple 2minute games, until heart rate habituation was observed. Habituation was defined as 3 of 4 consecutive HR responses that were within $5 \mathrm{bpm}$ of each other. The second task was the 2- min mental arithmetic challenge, followed by the 2-minute hand grip task. During the final challenge, the 2-min speech task, individuals were asked to discuss the topic of gun control. The speech task was presented one time (i.e., HR habituation was not assessed during the speech task). The highest average response during each task was calculated by subtracting baseline HR from HR 
observed during the task. The baseline value chosen was the lowest observed baseline HR value among the 4 baseline periods and was termed the initial baseline for the individual. The initial baseline was then subtracted from the HR values obtained during the tasks. The 6 individuals who demonstrated the highest average HR responses across the videogame, mental arithmetic, and hand grip tasks were asked to return for the HR feedback training sessions. All individuals were provided with extra-credit points in their psychology class for their participation in the pretraining session.

Videogame Training Sessions. The target HR goal during training was for the participant to demonstrate a HR response that was at most only $4 \mathrm{bpm}$ higher than the pre-training baseline HR observed during the pre-training session. Based upon the findings of Goodie and Larkin (1999) that individuals demonstrated an average $\mathrm{HR}$ response of $1.0 \mathrm{bpm}(\underline{\mathrm{SD}}=2.5 \mathrm{bpm})$ at post-training, the target HR response of $4 \mathrm{bpm}$ appeared to be an attainable goal for individuals in the current study. For the first training task (i.e., the videogame), the average HR from the last three pre-training presentations of the task (i.e., the initial heart rate response (IHR)) served as the target goal for that session and was entered into the Heart Rate Monitor (i.e., Target Value (1)). Subjects were instructed to reduce their HR below the target goal using the color feedback on the monitor, which showed subjects when they were above, equal, or below the target goal. For example, if a participant's HR during the pre-training presentation of the videogame averaged $85 \mathrm{bpm}$, the target goal was set at $85 \mathrm{bpm}$. If the individual's HR during the game was $89 \mathrm{bpm}$, the background turned red. If the HR level was at $85 \mathrm{bpm}$, the background turned white, and if the HR level was at $84 \mathrm{bpm}$ the screen turned blue.

To obtain HR data to adjust the training criterion, the HR during the last minute of the task was hand-counted. If the HR level had not reached the target goal, participants were 
instructed to reduce their HR and the task was again presented without changing the HR goal. If the HR level was equal to or below the target goal, the target goal was reduced. Eighty percent of the difference between the target goal and initial baseline was calculated, which was then added to the baseline value (Target value $(2)=(0.80 *(\mathrm{IHR}-$ mean initial baseline $))+$ mean initial baseline). The videogame task was again presented and participants were instructed to reduce their HR below the new target goal. Once the new target goal was reached during the last minute of the task, the next target goal was calculated using $60 \%$ of the initial response level (Target goal $(3)=(0.60 *($ IHR - mean initial baseline $))+$ mean initial baseline $)$. Upon reaching the third criterion level during the final minute of the task, a fourth goal at $40 \%$ of the original response to the task was added to the initial baseline established (Target goal $(4)=(0.40 *(\mathrm{IHR}-$ mean initial baseline)) + mean initial baseline). Participants continued to receive feedback with lowered criterion levels until they demonstrated a HR level that was 4 bpm or less above the initial baseline level. Once participants demonstrated a reduction of this size, the videogame was presented two more times with the same HR criteria (i.e., until participants demonstrated a HR level change of $4 \mathrm{bpm}$ or less during 3 consecutive presentations of the videogame). If the individual failed to meet the target goal after 3 presentations of the task, the goal was increased to the previous target goal.

As an example of the calculations used to determine target goals, suppose an individual demonstrated an average $\mathrm{HR}$ of $70 \mathrm{bpm}$ during the baseline period and a mean HR of $86 \mathrm{bpm}$ during the videogame challenge (i.e., an average HR response of $16 \mathrm{bpm}$ ) the initial criterion (Target value 1) entered into the Heart Rate Monitor would be $86 \mathrm{bpm}$. If the participant's HR was below 86 bpm during the last minute of the videogame challenge, then the second criterion would be set at $83 \mathrm{bpm}$ (i.e., $0.80 * 16=12.8$ and $12.8+70 \approx 83$ ). Once the second criterion was 
met during the last minute of the task, the third criteria would be set at $80 \mathrm{bpm}$. The fourth criterion would be $76 \mathrm{bpm}$ and the final criterion would be set at $74 \mathrm{bpm}$. The individual would be required to demonstrate a HR level of $74 \mathrm{bpm}$ during 3 presentations of the videogame prior to moving on to training with the next task.

To maximize compliance with the instructions and to enhance interest in the task during training, individuals received monetary rewards for their performance during the videogame and for successfully reducing their HR. For every 5 seconds that the HR was maintained below the target goal, 500 points were added to the final score. Likewise, 500 points were deducted from the score for every 5 seconds that participant's HR was above the criterion. The HR score was added to the performance score of the videogame, which is based on the distance that the individual traveled during the course of the game (i.e., hitting trees and using the brakes decreased distance traveled and resulted in a lower performance score). Each time the participant achieved a score that was 100 points higher than the previous score, the individual earned one dollar.

Each training session lasted a maximum of 2-hours. If the participant was unable to reach the target goal by the end of the 2-hour session, another training session was devoted to reaching the target goal during the videogame task. If the target goal was not reached during the second day of training, a final third session was devoted to HR feedback training during the videogame. Regardless of whether the target goal was reached on the third day of training, the next task (i.e., the mental arithmetic task) was presented during the subsequent session.

Immediate post-training assessment. After mastering HR reduction during the videogame task, or after three days of training during the videogame task, the three training tasks (i.e., the videogame, mental arithmetic, and hand grip tasks) were presented during an immediate 
post-training session, without HR feedback. A 4-minute rest period preceded each task. SUDs ratings were assessed at the end of each rest period and task. Participants were instructed to use the skills that they learned to keep their HR as low as possible during all tasks.

Training Sessions for the Mental Arithmetic and Hand Grip Tasks. The procedure for the mental arithmetic and hand grip training sessions was identical to the procedure used in videogame training. The monetary rewards during the mental arithmetic challenge were provided based on the number of correct calculations and the percentage of time the participant's HR was below the target goal during the task. During the hand grip challenge, the two minutes for the task were separated into 12 epochs. Participants accumulated points based on the number of epochs that they maintained $30 \%$ of their maximum grip. If at any point during an epoch the individual was unable to maintain $30 \%$ of their maximum grip, they did not receive credit for that epoch. During the mental arithmetic and hand grip tasks, each time the individual obtained a score that was 100 points higher than the previous score, he or she was awarded one dollar.

At the end of the mental arithmetic and hand grip training sessions, participants were presented with an immediate post-training session, during which the videogame, mental arithmetic, and hand grip challenge were presented without HR feedback. $\underline{\text { Short Delay Post-Training Assessment Session }}$

During the short delay post-training assessment session, the videogame, mental arithmetic, hand grip, and speech tasks were presented without HR feedback. The task order and procedure during the post-training session was the same as the task order and procedures employed during the pre-training assessment session except that the topic of the speech task was changed. As in the pre-training session, the presentation of each task was preceded by a 4-min resting period and instructions for completing the task. Participants were instructed to use the 
skills that they had learned to keep their HR as low as possible during each of the tasks. SUDs ratings were again assessed at the end of each rest period and task.

\section{Long Delay Post-Training Assessment Session}

One to two weeks following the post-training assessment session, individuals were asked to return one final time to the laboratory. Again, no HR feedback was provided and the tasks were presented in the same order as during the pre-training and short delay post-training assessment sessions. Participants were instructed to use the skills that they learned to keep their HR as low as possible during each of the tasks. SUDs ratings were assessed at the end of each rest period and task.

Following the long delay post-training assessment session participants were debriefed and received a $\$ 10$ payment for their participation, in addition to whatever amount of money that was earned during the course of the study.

\section{Results}

Due to the single-subject design of the study, inferential statistical analyses were not conducted. Instead, descriptive data and figures, are presented for each participant. The baseline HR levels, which were measured when participants were at rest prior to the beginning of each session are depicted in Figures 2 and 3. Upon inspection of these data, it is very clear that baseline HR values fluctuated greatly across sessions for all participants, perhaps reflecting different arousal levels that these students may have experienced during a typical semester. For example, if individuals were experiencing more significant stressors (e.g., upcoming exams, relationship problems), engaging in different sleeping patterns across session, or consuming substances that caused increases or decreases in HR, the baseline HR during one session, regardless of the amount of time allowed for habituation, would fluctuate across days. Baseline 
HR for even the most stable participant (i.e., 5), ranged from $64 \mathrm{bpm}$ to $80 \mathrm{bpm}$. Because of these fluctuating baselines, presentation of HR reactivity (i.e., change scores) for each participant across training phases and assessment sessions may not accurately portray their learning if change scores were calculated using baseline HR values obtained on that day. We chose to use a single estimate of initial baseline for each subject uniformly across all training and assessment sessions to calculate HR reactivity to all tasks. The initial baseline that was chosen was the lowest baseline HR observed during the pre-training assessment session. This was the most appropriate method to use because this baseline was used to calculate the target HR during HR feedback training (i.e., participants were asked to reduce their HR to within $4 \mathrm{bpm}$ of this baseline HR). Using the lowest pre-training baseline level to calculate HR reactivity to the tasks throughout the study allows the reader to see of how well each participant reduced his or her HR towards the target goal that was established using the initial baseline measure.

The HR responses to the tasks during pre-training, training, and post-training sessions are displayed in Figures 4 -9. The HR response to each presentation of the tasks is plotted. The HR response during each training session was calculated by subtracting the initial baseline HR from the pre-training session from the HR observed during each presentation of the task. The HR responses during the pre-training and post-training assessment sessions (i.e., immediate, short delay and long delay) were also calculated by subtracting the initial baseline HR from each HR measured during the task presentation.

Comparable SBP and DBP change scores for pre- and post-training assessment sessions are depicted in Figures $10-21$. The SBP and DBP response to each presentation of the tasks during the pre- and post-training assessment sessions is plotted. To be consistent with the HR data, a similar strategy was used to determine SBP and DBP responses to the challenges. That is, 
the lowest SBP observed during the baseline period of the pre-training session was determined to be the initial baseline SBP and was subtracted from all subsequent SBP readings. The same procedure was used to calculate DBP responses to the tasks.

The performance score, which was based on how well the participant performed during the task (i.e., without adjusting for success with heart rate feedback) is presented for each participant in Figures 22-27. The SUDs ratings associated with the task presentations during the pre-training, training, and post-training sessions are displayed in Figures 28-33.

Across all figures, responses to the videogame challenge are displayed with blue diamonds, responses to the mental arithmetic challenge are displayed with red squares, responses to the hand grip challenge are displayed with green triangles, and responses to the speech task are displayed with black circles.

\section{$\underline{\text { Pre-Training Assessment Session }}$}

\section{Videogame, Mental Arithmetic, Hand Grip, and Speech Challenges}

$\underline{\text { HR measures. }}$ The HR responses to pre-training tasks are presented in Figure 1 for all participants screened for the study and in the first panel (PT) of Figures 4-9 for the six individuals who completed the entire study. Participants 2, 4, 5, 6, 10, and 11 demonstrated the highest overall HR responses to the tasks. Participant 5 demonstrated somewhat lower responding to the videogame task compared to the other individuals; however, his HR responses to the other tasks were higher compared to the other participants. After participant 6 dropped out of the study, participant 13 was selected to replace him.

$\underline{\text { SBP measures. }}$ SBP reactions to the four tasks are shown in the first panel (PT) of Figures 10-15. Although there was considerable variability in SBP response to the three tasks during pre-training, there was no trend toward increasing or decreasing SBP responses. 
However, SBP response to the videogame was generally less than SBP responses to the other two tasks. SBP response to the speech challenge was typically greater than the other three tasks. DBP measures. DBP responses to the pre-training tasks are shown in the first panel (PT) of Figures 16-21. Consistent with SBP, considerable variability in DBP response was observed with no consistent increasing or decreasing trends. In general, DBP responses were greater for the hand grip challenge than the mental arithmetic challenge, which in turn was greater that the DBP response to the videogame. The speech task elicited DBP responses that were generally comparable to the hand grip challenge.

Behavioral measures. The performance scores of individuals during the pre-training session are presented in the first panel (PT) of Figures 22-27. .Regarding performance during the pre-training presentation of the videogame, four participants (i.e., 5, 10, 11, and 13) demonstrated performance scores that were quite stable, fluctuating less than 5000 points of each other (see Figures 22-27). Participants 2 and 4 demonstrated performance scores that were more variable, fluctuating between 7000 and 10000 points. Participants 4 and 11 (and participant 2 to some extent) demonstrated increasing trends in performance during the pre-training videogame task, whereas the remaining participants demonstrated no increasing or decreasing trends in performance.

Regarding performance during the pre-training mental arithmetic challenge, participants 2, 11, and 13 demonstrated relative stability of performance, with scores fluctuating 5000 or less points between presentations of the task. Participants 5 and 10 demonstrated performance scores that fluctuated as much as 9000 points between mental arithmetic task presentations, whereas participant 4 demonstrated performance scores that fluctuated 13000 points between mental arithmetic task presentations. Almost all participants showed some improvement in performance 
scores across the mental arithmetic presentations during the pre-training assessment session.

Regarding performance during the hand grip task, participants 10, 11, and 13

demonstrated very stable performance. Participants 2, 4, and 5 demonstrated performance scores that fluctuated as much as 12000 points, and all three cases showed decreased performance over time.

Subjective measures. The SUDs ratings of individuals during the pre-training session are presented in the first panel (PT) of Figures 28-33. In general, individuals reported the same pattern of SUDs ratings, with the hand grip task as most stressful, followed by the mental arithmetic challenge as the second most stressful, and the videogame task as the least stressful task. Three individuals (i.e., 5, 10, and 11) rated the speech task as stressful as the videogame task and the remaining 3 individuals (i.e., 2, 4, and 13) rated the speech task as stressful as the hand grip task. SUDs ratings were generally quite stable, with most of them fluctuating 20 points or less.

\section{$\underline{\text { Training Sessions }}$}

\section{Videogame Training}

HR measures. HR responses during the videogame training are presented in the second panel (VG Training) of Figures 4-9. Participants required between one to three sessions for videogame training, with two participants (i.e., 4 and 11) requiring one session, three participants (i.e., 2, 5, and 10) requiring two sessions, and one participant (i.e., 13) requiring three sessions. Every participant, except for participant 13, reduced their HR to the HR criteria during training trials. Interestingly, three participants (i.e., 4, 5, and 10) demonstrated HR responses to tasks at the end of training sessions that were similar to the HR response to the task at the beginning of the subsequent training session. That is, although at least one day had passed between training 
sessions, participants maintained the HR reductions observed during the previous session. In contrast to participants 4,5 , and 10, participants 2 and 13 demonstrated an increased HR response to the training task at the beginning of training sessions compared to the previous training session. That is, participants 2 and 13 did not appear to maintain HR reductions across time.

Behavioral measures. Performance scores (without HR adjustment) during the videogame training are presented in the second panel (VG Training) of Figures 22-27. Participants 2 and 13 demonstrated increasing trends in performance across the videogame training sessions. Participants 4, 5, 10, and 11 demonstrated no clear increasing or decreasing trends regarding performance across the videogame training sessions.

Subjective measures. SUDs ratings during the videogame training are presented in the second panel (VG Training) of Figures 28-33. Compared with SUDs ratings during the pretraining videogame task, participants 4, 5, and 10 demonstrated no increasing or decreasing trends, participants 2 and 11 demonstrated decreasing SUDs ratings, and participant 13 demonstrated increasing SUDs ratings across videogame presentations during training. Mental Arithmetic Training

HR measures. HR responses during the mental arithmetic task are presented in the third panel (MA Training) of Figures 4-9. Regarding HR changes during the mental arithmetic training sessions, participants demonstrated lower HR responses to the mental arithmetic challenge when provided with HR feedback. Two participants (i.e., 10 and 11) reduced their HR responses to the HR criteria level during the mental arithmetic training sessions. These two participants required one training session to reduce their HR responses to the mental arithmetic challenge, whereas the remaining four participants were presented the mental arithmetic 
challenge across three training sessions, but failed to reach the established criterion during that time. By the end of the mental arithmetic feedback training, four participants (i.e., 2, 4, 10, and 11) reduced their HR responses to the mental arithmetic challenge below the response levels observed during the pre-training sessions.

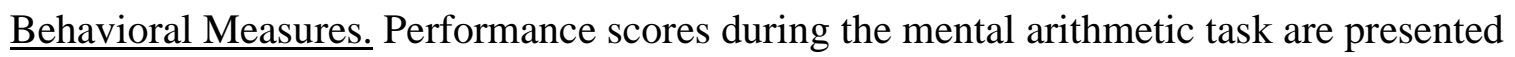
in the third panel (MA Training) of Figures 22-27. All six participants demonstrated performance scores that tended to increase across mental arithmetic presentations during training.

Subjective measures. SUDs ratings during the mental arithmetic task are presented in the third panel (MA Training) of Figures 28-33. Participants 5 and 10 reported SUDs ratings that were relatively consistent with the SUDs ratings reported during the pre-training mental arithmetic task. The remaining four participants reported lower SUDs levels during the mental arithmetic training sessions than the pre-training assessment session; however, participants 4 and 13 reported SUDs ratings that tended to increase within the training sessions, suggesting increased arousal or possibly frustration throughout the mental arithmetic training.

\section{Hand Grip Training}

Heart rate measures. HR responses during the hand grip task are presented in the fourth panel (HG Training) of Figures 4-9. One participant (i.e., 2) reduced her HR responses to the hand grip task to the heart rate criterion level. Although four of the participants (i.e., 4, 10, 11, and 13) demonstrated reduced HR responses to the hand grip task within individual training sessions, they did not exhibit a reduction in HR responses across sessions, preventing them from reaching the HR criterion. Finally, one participant (i.e., 5) showed steady increases in HR responsivity with training. 
Behavioral measures. Performance scores during the hand grip task are presented in the fourth panel (HG Training) of Figures 22-27. Regarding performance across the hand grip training sessions, participants 5, 10, 11, and 13 demonstrated performances that were relatively stable, whereas participant 4 demonstrated a decreasing trend in performance and participant 2 demonstrated an increasing trend during the first training session and a stable performance during the second training session.

Subjective measures. SUDs ratings during the hand grip task are presented in the fourth panel (HG Training) of Figures 28-33. Across the handgrip training sessions, participants 2, 5, 10, and 11 reported SUDs ratings that remained stable across training. Participant 4 reported increasing SUDs ratings within each of the three handgrip training sessions. Although no obvious trend was observed, participant 13 reported SUDs levels that fluctuated approximately 30 points between presentations of the handgrip task.

\section{Post-Training Sessions}

$\underline{\text { Immediate Post-Training }}$

HR responses. HR responses to the tasks during the immediate post-training assessments are labeled with an "I" and presented as a sub-section at the end of each training session within the second, third, and fourth panels in Figures 4-9. Examining the HR responses to the videogame task during the videogame immediate post-training assessment revealed that five participants (i.e., 4, 5, 10, 11, and 13) demonstrated HR responses that were equivalent to the last three HR responses during the videogame training session, whereas participant 2 demonstrated a higher HR response to the immediate post-training assessment videogame presentation compared to the last three videogame presentations during feedback training. Surprisingly, though, participant 2's HR response to the videogame was lower again following training in mental 
arithmetic and hand grip challenges. For the other participants, one participant (i.e., 13)

maintained her HR response reduction to the videogame challenge across all immediate posttraining periods; one participant (i.e., 11) maintained his HR reduction following the mental arithmetic training trials, but showed an increasing trend following the hand grip training trials; and the remaining three participants (i.e, 4, 5, and 10) showed an increased HR reactivity to the videogame challenge following the mental arithmetic and hand grip training periods in contrast to the immediate assessment following videogame training trials.

Regarding HR responses to the mental arithmetic challenge across immediate posttraining sessions, four participants (i.e., 2, 4, 5, and 13) demonstrated comparable HR responses to the mental arithmetic challenge following videogame training than pre-training. For these four participants, training in HR reduction to the videogame challenge did not transfer to the mental arithmetic task. Participants 10 and 11, however, exhibited a reduction in HR response to the mental arithmetic challenge following training on the videogame task. During the mental arithmetic immediate post-training assessment (i.e., following HR feedback training during the mental arithmetic challenge), four participants (i.e., 2, 5, 11, and 13) demonstrated HR responses to the mental arithmetic task that were similar to the HR responses demonstrated during the final presentations of the preceding training session. Of these, only participants 5 and 13 maintained their reduced HR reactivity to the mental arithmetic challenge during the hand grip immediate post-training assessment.

Regarding HR responses to the hand grip challenge across immediate post-training assessments, all participants exhibited decreased HR responses to the hand grip task during the videogame immediate post-training assessment. Therefore, all participants showed some generalization to the hand grip task following training using the videogame. Although HR 
responses to the hand grip tended to increase slightly during the mental arithmetic immediate post-training assessment (i.e., 4, 5, 10, 11, and 13), the levels still tended to be lower than those observed for hand grip at pre-treatment. During the hand grip immediate post-training assessment, all participants demonstrated HR responses to the hand grip task that were similar to the responses observed during the final presentations of the hand grip task during the training sessions and lower than HR responses observed during the pre-treatment hand grip task.

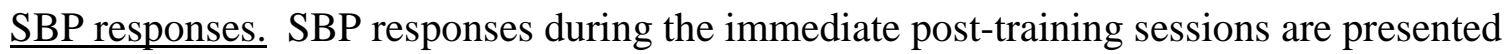
in the panels labeled "VG-, MA-, and HG- Post-training" in Figures 10-15. SBP responses to the videogame during the videogame immediate post-training assessment were equivalent to the SBP responses observed during the pre-training videogame for four participants (i.e., 2, 4, 10, and 13), lower for one participant (i.e., 5) and higher for one participant (i.e., 11). During the mental arithmetic immediate post-training assessment, SBP responses to the videogame presentation were equivalent to SBPs observed during the pre-training videogame presentation for participants 5, 10,11 , and 13 and lower for participants 2 and 4. During the hand grip immediate post-training session, SBP responses to the videogame task were equivalent to pretraining levels for three participants (i.e., 5, 10, and 13), lower for participants 2 and 4, and higher for participant 11.

SBP responses to the mental arithmetic task during the videogame immediate posttraining assessment were equivalent to the SBP responses to the mental arithmetic challenge observed during the pre-training for four participants (i.e., 2, 5, 10, and 11), and lower for two participants (i.e., 4 and 13). During the mental arithmetic immediate post-training assessment, SBP responses to the mental arithmetic task were equivalent to pre-training mental arithmetic SBP levels for two participants (i.e., 11 and 13), lower for three participants (i.e., 2, 4, and 5) and 
higher for participant 10. During the hand-grip immediate post-training assessment SBP responses to the mental arithmetic task were equivalent to pre-training SBP responses to the mental arithmetic task for three participants (i.e., 2, 11 and 13), lower for two participants (i.e., 4 and 5) and higher for one participant (i.e., 10).

SBP responses to the hand grip task during the videogame immediate post-training assessment were equivalent to SBP responses to the pre-training hand-grip task for almost all participants (i.e., 2,4, 5, 10, and 11). Participant 13 evidenced reduced SBP response to the hand grip task following videogame training. During the mental arithmetic immediate post-training assessment, SBP responses to the hand-grip task were equivalent to pre-training hand grip levels for most participants (i.e., 2, 4, 5, 11 and 13) and slightly higher for participant 10. During the hand grip immediate post-training assessment, SBP responses to the hand grip task were equivalent to pre-training SBP levels for five participants (i.e., 2, 5, 10, 11, and 13) and slightly lower for one participant (i.e., 4).

DBP responses. DBP responses during the immediate post-training sessions are presented in the panels labeled "VG-, MA-, and HG- Post-training" in Figures 16-21. DBP responses to the videogame during the immediate videogame post-training session were equivalent to pre-training for four participants (i.e., 2, 4, 5, and 11), higher for participant 10, and lower for participant 13. DBP responses to the videogame during the mental arithmetic immediate post-training session were equivalent to pre-training DBP responses to the videogame for four participants (i.e., 2, 4, 11 and 13), lower for participant 5, and higher for participant 10. DBP responses to the videogame task during the hand grip immediate post-training session were equivalent to pretraining DBP responses for four participants (i.e., 2, 4, 5, and 11) and higher for participants 10 and 13. 
DBP responses to the mental arithmetic task during the videogame immediate posttraining assessment were equivalent to pre-training DBP responses to the mental arithmetic task for five participants (i.e., 2, 4, 10, 11, and 13) and slightly lower for participant 5. DBP responses to the mental arithmetic challenge during mental arithmetic immediate post-training session were equivalent to DBP responses to the mental arithmetic task during pre-training for three participants (i.e., 10, 11, and 13) and lower for three participants (i.e., 2, 4, and 5). DBP responses to the mental arithmetic challenge during the hand grip immediate post-training session were equivalent to DBP responses to the pre-training mental arithmetic challenge for four participants (i.e., 2, 4, 11, and 13) and lower for participant 5, and higher for participant 10.

DBP responses to the hand grip task, during the videogame immediate post-training assessment were equivalent for four participants (i.e., 5, 10 ,11, and 13) and lower for two participants (i.e., 2 and 4). DBP responses to the hand grip task during the mental arithmetic immediate post-training session were equivalent to pre-training DBP responses to the hand grip task for three participants (i.e., 4, 11 and 13) and lower for three participants (i.e., 2, 5, and 10). DBP responses to the hand grip task during the hand grip immediate post-training assessment were equivalent to pre-training DBP responses during the hand grip task for participant 13 and lower for five participants (i.e., 2, 4, 5, 10 and 11).

Behavioral responses. Performance scores during the immediate post-training assessments are labeled with an "I" and presented as a sub-section at the end of each training session within the second, third, and fourth panels in Figures 22-27. Regarding performance during the videogame across the three immediate post-training assessments (i.e., videogame, mental arithmetic, and hand grip), five participants (i.e., 2, 4, 5, 10, and 11) demonstrated performance scores that were equivalent to the average performance score obtained during the 
pre-training session. Participant 13 demonstrated videogame performance scores across the 3 immediate post-training assessments that were slightly higher than the performance score obtained during the pre-training videogame presentations.

Regarding performance during the mental arithmetic challenge, all participants demonstrated increasing performance scores across the three immediate post-training assessments. Participant 5 demonstrated the greatest increase in performance (i.e., 189\%) between the videogame and the hand grip immediate post-training sessions.

Regarding performance during the hand grip task, five participants (i.e., 2, 4, 10, 11, and 13) demonstrated consistent performance across the three immediate post-training assessments and performance scores that were equivalent to the average performance demonstrated during the pre-training assessment. One participant (i.e., 5) demonstrated an improved performance across the immediate post-training assessments.

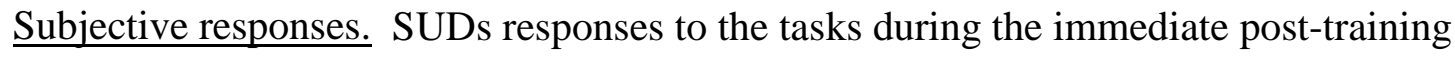
assessments are labeled with an "I" and presented as a sub-section at the end of each training session within the second, third, and fourth panels in Figures 28-33. Regarding SUDs responses to the videogame across the immediate post-training assessments, participants reported SUDs levels that were lower than or equivalent to the lowest SUDs ratings reported following the pretraining videogame. Participants 2, 4, 5, 10, and 11 videogame SUDs ratings remained consistent across the three immediate post-training assessments, whereas participant 13's SUDs responses to the videogame decreased from the videogame to the hand grip immediate posttraining assessments.

Regarding SUDs responses to the mental arithmetic across immediate post-training assessments, individuals reported lower SUDs ratings during the immediate post-training 
assessments than reported during the pre-training mental arithmetic challenge. Comparing the three immediate post-training assessments, three participants (i.e., 5, 10, and 13) demonstrated a decreasing trend, whereas three participants (i.e., 2, 4, and 11) demonstrated stable SUDs rating across immediate post-training assessments.

Regarding SUDs responses to the hand-grip task, five participants (i.e., 2, 4, 5, 10, and 11) reported SUDs ratings that were lower than the SUDs ratings reported during the pre-training hand grip task presentations, whereas participant 13 reported SUDs ratings during the videogame and mental arithmetic post-training assessments that were as high as the highest SUDs rating reported during the pre-training hand grip task. Five participants (i.e., 2, 4, 5, 10, and 11) reported consistent SUDs ratings across the three immediate post-training assessments, whereas participant 13 demonstrated a lower SUDs rating during the hand grip immediate post-training assessment compared to the videogame and mental arithmetic immediate post-training assessments.

\section{$\underline{\text { Short Delay Post-Training Assessment }}$}

HR responses. HR responses during the short delay post-training assessment are presented in the fifth panel labeled SD in Figures 4-9. Compared to the HR responses observed during the pre-training session, only participant 5 demonstrated lower HR responses to all three training tasks during the short delay post-training assessment.

Regarding HR responses to the videogame challenge, only participant 5 demonstrated HR responses to the videogame that were lower than the HR responses observed during the pretraining session. Participants 4 and 11 demonstrated HR responses that were roughly equivalent to the pre-training response, and the remaining three participants (i.e., 2, 10, and 13) demonstrated higher HR responses to the videogame compared to the pre-training levels. Except 
for participant 5, the participants demonstrated HR responses to the videogame that were higher than the HR responses to the videogame observed during the final videogame training period.

Regarding HR responses to the mental arithmetic challenge, only participant 5 demonstrated HR responses to the mental arithmetic challenge that were lower than the HR responses to the mental arithmetic challenge observed during the pre-training session.

Participants 2, 4, 10, and 11 demonstrated HR responses to the mental arithmetic challenge that were roughly equivalent to the HR responses to the mental arithmetic challenge that were observed during the pre-training session. Participant 13 demonstrated a higher HR response to the short delay post-training mental arithmetic challenge compared to the pre-training HR responses to the mental arithmetic challenge. Compared to the HR responses to the mental arithmetic challenge observed during the final mental arithmetic training session, five participants (i.e., 2, 4, 10, 11, and 13) demonstrated higher HR responses to the short delay posttraining mental arithmetic challenge. Only participant 5 demonstrated a lower HR response to the short delay post-training assessment compared to the final HR responses to the mental arithmetic training challenges.

Regarding HR responses to the hand grip task, four participants (i.e., 2, 4, 10 and 11) demonstrated HR responses that were roughly equivalent to the HR responses to the hand grip task observed during the pre-training session. Only participants 5 and 10 demonstrated a HR response to the short delay post-training hand grip task that was lower than the HR response to the hand grip task demonstrated during the pre-training assessment. Half of the participants (i.e., 2, 5, and 13) demonstrated HR responses to the hand grip task that were equivalent to HR responses observed during the hand grip training session. The remaining three participants (i.e., 4, 10, and 11) demonstrated HR responses to the short-delay post training session that were 
higher than the HR responses observed during the hand grip training session.

Regarding the speech task, compared to the pre-training HR responses to the speech task, four participants (i.e., 2, 4, 5, and 11) demonstrated somewhat lower HR responses; participant 13 demonstrated an equivalent HR response, and participant 10 demonstrated a higher HR response to the short delay post-training assessment.

$\underline{\text { SBP responses. }}$ SBP responses during the short delay post-training assessment are presented in the panel labeled "Short Delay Post-Training" in Figures 10-15. SBP responses to the short-delay post-training videogame task were equivalent to pre-training SBP responses to the videogame for four participants (i.e., 2, 5, 10 and 13), lower for participant 4, and higher for participant 11.

SBP responses to the short-delay post-training mental arithmetic challenge were equivalent to SBP levels during the pre-training mental arithmetic for participants 11 and 13, lower for three participants (i.e., 2, 4, and 5), and higher for participant 10.

SBP responses to the short-delay post-training hand grip challenge were equivalent to pre-training SBP responses to the hand grip challenge for three participants (i.e., 10, 11, and 13) and lower for three participants (i.e., 2, 4, and 5).

SBP responses to the short-delay post-training speech challenge were equivalent to pretraining SBP levels for two participants (i.e., 10 and 13) and lower for four participants (i.e., 2, 4, 5 , and 11).

DBP responses. DBP responses during the short delay post-training assessment are presented in the panel labeled "Short Delay Post-training" in Figures 16-21. DBP responses to the short-delay post-training videogame were equivalent to DBP responses to the pre-training videogame for four participants (i.e., 2, 5, 10, and 13), higher for participant 4, and lower for 
participant 11.

DBP responses to the short-delay post-training mental arithmetic task were equivalent to DBP responses to the pre-training mental arithmetic task for four participants (i.e., 2, 4, 11, and 13) and lower for two participants (i.e., 5 and 10).

DBP responses to the short-delay hand grip challenge were equivalent to DBP responses to the pre-training hand-grip challenge for one participant (i.e., 13) and lower for 5 participants (i.e., 2, 4, 5, 10, and 11).

DBP responses to the short-delay post-training speech challenge were equivalent to pretraining DBP responses to the speech challenge for four participants (i.e., 2, 4, 11, and 13), lower for participant 5, and higher for participant 10.

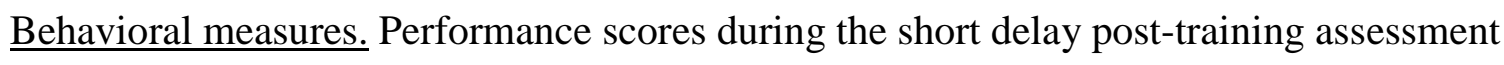
are presented in the fifth panel labeled SD in Figures 22-27. Regarding performance during the short delay post-training videogame task, five participants (i.e., 2, 4, 5, 10, and 11) demonstrated scores that were equivalent to the performance score obtained during the pre-training session and participant 13 demonstrated a score that was slightly higher than the pre-training performance. All participants demonstrated performance scores that were roughly equivalent to the scores obtained during the final presentations of the videogame during the training session.

During the short delay post-training mental arithmetic task, five participants (i.e., 4, 5, 10, 11, and 13) demonstrated higher performance scores compared to the pre-training performance score and participant 2 demonstrated a performance score that was equivalent to the pre-training performance score. Compared to the performance scores obtained during training, all participants demonstrated performance scores during the short delay post-training session that were equivalent to those obtained during the final presentations of the mental arithmetic task 
during training.

During the short delay post-training hand grip task, all participants demonstrated performance scores that were roughly equivalent to the scores obtained during the pre-training session. Similarly, performance for all participants during the short delay post-training hand grip task was equivalent to the performance demonstrated during the hand grip training session.

No behavioral data were obtained for the speech task.

In sum, the videogame and hand grip scores remained fairly stable from beginning to end, while scores on the mental arithmetic task tended to improve.

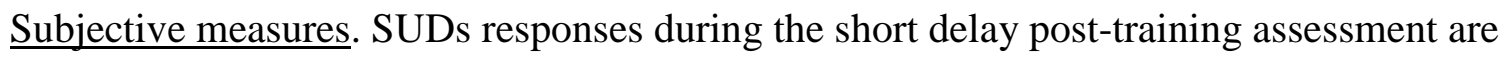
presented in the fifth panel labeled SD in Figures 28-33. All participants reported a decrease in SUDs during all three training tasks delivered during the short delay post-training period compared to the average SUDs ratings reported during the pre-training period.

Participants 2, 4, 11, and 13 reported a decrease in SUDs during the short delay posttraining speech task compared to the average SUDs ratings reported during the pre-training session speech task. Participant 5 reported the same SUDs rating during both pre- and posttraining and participant 10 reported a slightly increased SUDs rating during the short delay posttraining speech task compared to the average SUDs ratings reported during the pre-training session speech task.

Long Delay Post-Training Assessment

$\underline{\text { HR responses. }}$ HR responses observed during the long delay post-training assessment are displayed in the sixth panel labeled LD in Figures 4 -9. All participants demonstrated HR responses to the videogame that were equivalent to the HR responses observed during the pretraining videogame. Compared to the HR responses to the videogame observed during the final 
training presentations of the videogame, four participants (i.e., 4, 5, 10, and 11) demonstrated higher HR responses and two participants (i.e., 2 and 13) demonstrated equivalent $\mathrm{HR}$ responses to the long delay post-training videogame.

Only participants 2 and 11 demonstrated HR responses to the mental arithmetic challenge that were lower than the HR response observed during the pre-training session. Three participants (i.e., 5, 10, and 13) demonstrated $\mathrm{HR}$ responses that were equivalent to pre-training measures of $\mathrm{HR}$ response to the mental arithmetic task and 1 participant (i.e., 4) demonstrated a HR response to the mental arithmetic challenge that was higher than the HR responses to the pretraining mental arithmetic challenge.

Regarding the hand grip task, five participants (i.e., 2, 4, 10, 11, and 13) demonstrated HR responses that were lower than the responses observed during the pre-training session. Participant 5 demonstrated a HR response to the long-delay hand grip task that was equivalent to the HR response observed during the pre-training session.

Three participants (i.e., 2, 4, and 11) demonstrated a HR response to the speech task that was lower than the pre-training HR response to the speech task. For the remaining three participants (i.e., 5, 10, and 13) HR response to the speech task at the long delay post-training was equivalent to HR response at pre-treatment.

Blood pressure responses. SBP responses during the training sessions are presented in the panel labeled "Long Delay Post-training" in Figures 10-15. SBP responses to the long delay post-training videogame were equivalent to SBP responses to the pre-training videogame for five participants (i.e., 4, 5, 10, 11, and 13) and lower for participant 2.

SBP responses to the long delay post-training mental arithmetic task were lower than the SBP responses to the pre-training mental arithmetic challenge for five participants (i.e., 2, 4, 5, 
11, and 13) and higher for participant 10.

SBP responses to the long delay hand grip task were equivalent to the SBP responses to the pre-training hand grip task for three participants (i.e., 5, 10, and 13) and lower for three participants (i.e., 2, 4 and 11).

SBP responses to the long delay speech task were equivalent to the SBP responses to the pre-training speech challenge for two participants (i.e., 10 and 13) and lower for four participants (i.e., 2, 4, 5, and 11).

DBP responses. DBP responses during the training sessions are presented in the panel labeled "Long Delay Post-training" in Figures 16-21. DBP responses to the long delay posttraining videogame were equivalent to the DBP responses to the pre-training videogame for five participants (i.e., 2, 4, 10, 11, and 13) and lower for participant 5.

DBP responses to the long delay post-training mental arithmetic challenge were lower than the DBP responses to the pre-training mental arithmetic for five participants (i.e., 2, 4, 5, 10, and 11) and equivalent for participant 13.

DBP responses to the long delay post-training hand-grip challenge were equivalent to DBP responses to the pre-training hand grip challenge for two participants (i.e., 10 and 13) and lower for four participants (i.e., 2, 4, 5, and 11).

DBP responses to the long delay post-training speech task were equivalent to DBP responses to the pre-training speech task for two participants (i.e., 10 and 13) and lower for 4 participants (i.e., 2, 4, 5, and 11).

Behavioral Measures. Behavioral responses observed during the long delay post-training assessment are displayed in the sixth panel labeled LD in Figures 22-27. Regarding performance during the long delay post-training videogame task, all participants demonstrated a stable 
performance from pre-treatment, except for participant 13 who demonstrated an increase in performance compared to the pre-training videogame task. In brief, all participants maintained their performance on the videogame task from the short- to long-delay post assessment period.

During the long delay post-training mental arithmetic task, all participants demonstrated an increase in performance compared to the pre-training mental arithmetic task. Congruent with findings for the videogame task, all participants maintained their performance gains from the short- to the long-delay assessment periods.

During the long delay post-training hand grip task, participant 11 demonstrated a small decrease in performance, whereas the rest of the participants demonstrated increased performance compared to the pre-training hand grip task.

Again, no measures of performance were obtained for the speech task.

Subjective measures. SUDs responses observed during the long delay post-training assessment are displayed in the sixth panel labeled LD in Figures 28 -33. Participants reported a decrease in SUDs during all three training tasks presented during the long delay post-training period compared to the average SUDs ratings reported during the pre-training session period.

Participants 5 and 10 reported the same SUDs level during the long delay post-training speech task compared to the average SUDs ratings reported during the pre-training session speech task. Participants 2, 4, 11, and 13 demonstrated lower SUDs ratings during the long delay post-training speech task.

\section{Discussion}

The present study replicates previous findings (Goodie \& Larkin, 2001; Larkin et al., 1989; Larkin et al., 1990; Larkin, Zayfert, Veltum, \& Abel, 1992) suggesting that individuals can reduce their HR when provided with HR feedback during the presentation of a videogame task.. 
Overall, the data replicated the findings of Goodie and Larkin. That is, individuals reduced their HR responses to a videogame task with HR feedback and then successfully reduced their HR responses to the videogame task, without HR feedback present, during an immediate posttraining session. Similarly, performance during the videogame remained relatively stable across pre-training, training, and post-training sessions.

The present study extends previous data by demonstrating that individuals can also reduce their HR when provided with HR feedback during a mental arithmetic task and a hand grip task. All participants generally demonstrated decreased HR responses to the mental arithmetic challenge within each training session and five participants (i.e., 2, 4, 10, 11, and 13) demonstrated decreased HR responses to the hand grip task. The present study partially replicated findings (Goodie \& Larkin, 1999; Larkin et al., 1989; Larkin et al., 1990; Larkin, Zayfert, Veltum, \& Abel, 1992) that suggested that individuals can lower their HR response when HR feedback is not provided. Five participants demonstrated lowered HR responses to the videogame task during the videogame immediate post-training assessments, four participants demonstrated lower responses to the mental arithmetic challenge during the immediate posttraining mental arithmetic assessment, and all participants demonstrated lowered HR responses to the hand grip task during the hand grip immediate post-training session. Overall, however, the study failed to support previous data (Larkin et al., 1992; Sharpley, 1994) suggesting that individuals can learn to apply HR reduction skills beyond an immediate post-training assessment. Responses to the tasks during the short-delay and long-delay tasks were generally higher than those observed during the final training session for each task and approached, or were equivalent to, the HR responses observed during the pre-training assessment.

In general, most blood pressure responses to tasks during the immediate post-training 
sessions were equivalent to pre-training blood pressure responses, where as during the short- and long-delay post-training sessions, blood pressure responses tended to be mixed (i.e., either equivalent or lower) compared to pre-training blood pressure responses.

Consistent with the previous literature (Goodie \& Larkin, 1999; Larkin et al., 1990, 1992), performance during the tasks remained constant or improved across presentations of the tasks (i.e., the delivery of HR feedback did not interfere with task performance). Similarly, across training sessions and post-training sessions, SUDs ratings remained relatively similar across task presentations, although SUDs ratings were generally lower than those observed during the pre-training assessment. The following sections focus on findings pertaining to HR, SBP, DBP, behavioral, and subjective responses in this study, including a description of some of the limitations of the study and suggestions for future research.

\section{$\underline{\text { Training Sessions }}$}

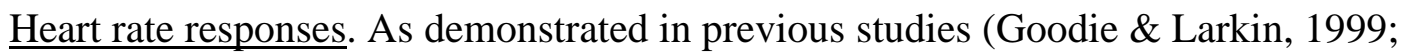
Larkin et al., 1989; Larkin et al., 1990; Larkin, Zayfert, Veltum, \& Abel, 1992), participants decreased HR responding to the videogame during the videogame training sessions and 5 participants (i.e., 2, 4, 5, 10, and 11) reached the target HR criteria. During the immediate posttraining session four of these participants (i.e., 4, 5, 10, and 11) demonstrated HR responses to the videogame that were both similar to the HR responses observed during the final presentation of the videogame during the training task and lower than the HR responses observed during the pre-training assessment.

The magnitude of the reduction in HR to the videogame appears smaller in the present study than HR reductions observed in previous studies, which is likely to be related to several factors. First, previous studies (i.e, Goodie \& Larkin, 1999; Larkin et al., 1989; Larkin et al., 
1990; Larkin, Zayfert, Veltum, \& Abel, 1992) assessed initial HR response to the videogame on the same day as training and the baseline levels that were used to calculate change scores remained constant between the pre-training, training, and post-training sessions. Because baseline HR levels varied greatly between sessions, which occurred on different days, using a constant baseline level from the pre-training assessment minimized the depiction of success among participants in reducing their HR. In fact, when the daily baseline level was used to calculate change scores, instead of the initial baseline value, participants exhibited what appear to be more dramatic declines in HR during training (see Appendix E). A second factor that may have contributed to the lower magnitude of HR reduction in the current study pertains to the fact that during the pre-training sessions, the tasks were presented multiple times until HR habituation was observed. Therefore, unlike previous studies (Goodie \& Larkin, 1999; Larkin et al., 1989; Larkin et al., 1990; Larkin, Zayfert, Veltum, \& Abel, 1992), participants were less likely to demonstrate a lowered HR response during the first training session that was due to both habituation and reductions associated with HR feedback training.

Although previous researchers (Goodie \& Larkin, 1999; Larkin, Zayfert, Abel, \& Veltum, 1992) used the mental arithmetic challenge to examine transfer of HR feedback training, the present study was the first to examine using the mental arithmetic challenge as a HR feedback-training task. Although only two participants (i.e., 10 and 11) reached the target HR criterion within training sessions, all participants demonstrated a decreased HR response to the mental arithmetic challenge during training. These data suggest that although the mental arithmetic challenge involved a different behavioral topography (i.e., auditory, cognitive, and verbal parameters) in comparison to the videogame (i.e., visual and motor parameters), individuals were able to reduce their HR responses during the presentation of such tasks. 
The hand grip task also had not been used in previous studies of HR feedback training. However, individuals generally were able to reduce their HR response to the hand grip challenge during the training session, although in several instances HR responses tended to increase during the later task presentations within a HR feedback training session (e.g., participant 2 and 3's first hand grip training session). Again, these data suggest that individuals were able to learn to reduce their HR responses to a task that involved a different behavioral topography (i.e., muscular endurance and physical response parameters) than the behaviors involved in the videogame challenge.

Behavioral Responses. Consistent with previous research (Goodie \& Larkin, 1999; Larkin et al., 1990, 1992), performance during the videogame remained stable (i.e., participants $4,5,10$, and 11) or improved slightly (i.e., participants 2 , and 13) across presentations of the videogame during training trials. Although one previous study (i.e., Larkin et al., 1989) found that a group that did not receive feedback performed better than a group that did receive feedback, most other studies (Goodie \& Larkin, 1999, Larkin et al. 1990, Larkin et al., 1992) have not found differences in behavioral performance during training using very similar protocols. Although there was no control group in the current study, the absolute values and trends in performance were consistent with previous research.

Performance during the mental arithmetic challenge remained stable for two participants (i.e., 2 and 11), but increased across time for the remaining four participants (i.e., participants 4 , 5, 10, and 13). Because previous research has not used mental arithmetic during the training sessions, there are no previous studies to compare the performance scores; thus performance can only be compared to videogame performance in this and previous studies. One explanation that participants demonstrated an increase in performance in mental arithmetic across training 
sessions, is that the mental arithmetic challenge, unlike the videogame or hand grip challenge, did not have a ceiling on the performance score. During the mental arithmetic challenge, participants could increase their performance by correctly subtracting more numbers, whereas during the other two tasks, the maximal performance score could be obtained early in training. Therefore, the lack of a ceiling effect during the mental arithmetic challenge, unlike the videogame and hand grip challenge, allowed individuals to continually improve their performance scores across training trials.

Within the hand grip training sessions, individuals generally maintained consistent performance across and within the training sessions, except participant 4 who demonstrated decreased performance scores within the training sessions. In order to maintain comparable levels of performance to the hand grip challenge, the number of presentations of the hand grip challenge within each training session was fewer than the number of presentations of the videogame or mental arithmetic challenge. Individuals were also given approximately 5 minutes to recover after each presentation of the hand grip task to give the participants' muscles an opportunity to recover from the task. Despite this difference in timing, comparable effects were observed and no deterioration in performance was observed.

Overall the performance scores from the videogame, mental arithmetic, and hand grip challenge are similar to findings of previous studies (Goodie \& Larkin, 1999; Larkin et al., 1990, 1992), suggesting that HR feedback training does not significantly interfere with performance during environmental challenges that involve varying behavioral topographies.

$\underline{\text { Subjective responses. }}$ SUDs ratings during the videogame challenge were inconsistent across participants; compared to pre-training, SUDs ratings of three individuals (i.e., 4, 5, and 10) demonstrated no trends, the ratings of participants 2 and 11 demonstrated decreasing trends, 
and the ratings of participant 13 demonstrated an increasing trend in SUDs ratings during training. Those who demonstrated no stable pattern in SUDs ratings, or who demonstrated decreasing trends, required 1 or 2 training sessions to meet the target HR, whereas participant 13 never met the target HR. Therefore participant 13's increasing arousal score may reflect increased frustration associated with her inability to reduce HR to the target level.

SUDs ratings during the mental arithmetic challenge also demonstrated a mixed pattern, with 2 individuals (i.e., 5 and 10) reporting consistent SUDs ratings, 2 participants (i.e., 2 and 11) reporting lower SUDs ratings, and 2 participants (i.e., 4 and 13) reporting higher SUDs ratings during training compared to the pre-training SUDs ratings during the mental arithmetic challenge. Again, two participants who did not meet the HR criterion (i.e., 4 and 13) demonstrated increased SUDs ratings during training, possibly reflecting increased frustration associated with not reaching the HR criterion.

SUDs ratings for the hand grip task also failed to demonstrate a consistent pattern. Five participants (i.e., 2, 5, 10,11, and 13) demonstrated no increasing or decreasing trends. Participant 4 was the only participant to demonstrate increasing trends in SUDs ratings within a training session. Participants with more "stable" SUDs responses completed fewer presentations of the hand grip task within a session.

Overall, the SUDs data suggest that no individual became disinterested in the tasks during the training sessions despite the multiple presentations of the tasks. Overall individuals generally demonstrated consistent or increasing SUDs responses, which suggested that the participants found the tasks to be consistently challenging across presentations. It may also be noteworthy that, except for participant 4 , participants tended to rate the tasks as similarly stressful. Late in the training sessions, participant 4 tended to rate the mental arithmetic and 
hand grip challenges as more stressful than the videogame challenge.

\section{$\underline{\text { Post-Training Assessments }}$}

Heart rate responses. As demonstrated in previous studies (Goodie \& Larkin, 1999;

Larkin et al., 1989; Larkin et al., 1990; Larkin, Zayfert, Veltum, \& Abel, 1992), five participants (i.e., 4, 5, 10, 11, and 13) reduced their HR responses to the videogame during an immediate post-training assessment (i.e., no HR feedback was present) to a level that was equivalent to the HR responses observed at the end of the training session. However, the HR response was equivalent to the HR response observed during the pre-training session for two (i.e., participants 5 and 13) of these five participants. During subsequent post-training sessions (i.e., after training in mental arithmetic and hand grip), participants demonstrated an increasing trend in their HR responses to the videogame task. These data suggested that individuals failed to retain the skills to lower HR during the videogame task that had been learned during the training session.

Four participants (i.e., 2, 5, 11, and 13) demonstrated HR responses to the immediate post-training mental arithmetic assessment that was equivalent to the final HR responses observed during the mental arithmetic training session. However, only two of these participants (i.e., 2 and 11) demonstrated HR responses during the immediate post-training mental arithmetic challenge that were lower than the HR responses to the mental arithmetic challenge observed during the pre-training assessment. Similar to the HR responses observed during the videogame challenge, participants generally demonstrated increasing HR responses to the mental arithmetic challenge across subsequent post-training sessions. These HR responses often were equivalent or higher than the HR responses observed during the pre-training assessment.

HR responses to the immediate post-training hand grip task were equivalent to the HR responses to the hand grip task observed during the final training session, and these responses to 
the hand grip challenge were lower than those observed during the pre-training session.

Interestingly, HR responses to the hand grip task were generally higher during the short delay post training assessment, but then returned to reduced HR response levels during the long delay post-training assessment. Therefore, unlike the HR responses observed during the videogame and mental arithmetic challenge, there was some limited evidence that participants appeared to maintain the skills that they had learned regarding HR reduction during the hand grip challenge.

During the speech task, the task designed to examine transfer of HR feedback training, three participants demonstrated lower HR responses during the short and long delay posttraining assessment compared to the pre-training assessment (i.e., 2, 4, and 11). Therefore, the data suggest that some individuals may have transferred skills for reducing HR to a "novel" task. However, given that most participants (i.e., 2, 4, 5, 10, and 13) failed to demonstrate consistent reductions in HR to the training tasks across post-training assessments, it is unlikely that participants could apply HR reduction skills to the "novel" speech task without HR feedback present. Therefore, the reductions in HR response to the speech task that occurred probably represented habituation to the task. Unlike the training tasks, habituation to the speech task did not occur during pre-training; therefore, it is more difficult to rule-out habituation as a causal factor for the HR reduction observed during the speech task at post-training.

Overall, the post-training HR response data suggests that most individuals, except for participant 11 , demonstrated little evidence that they could apply the HR reduction skills that they had learned during training to post-training sessions, other than the immediate post-training session. This data is consistent with the results reported by Goodie and Larkin (1999) who found that individuals could reduce HR responses to a challenge during training and apply those skills during an immediate post-training session, but not apply those skills to a novel task (i.e., mental 
arithmetic challenge) at post-training.

The data regarding the transfer of HR feedback training are inconsistent with the data reported by Larkin, Zayfert, Abel, and Veltum (1992) and Sharpley (1994). There are several differences between the present study and these two studies (Larkin, Zayfert, Abel, \& Veltum; Sharpley) that may contribute to the discrepant results observed among these studies. Although Larkin et al. used a similar training procedure using the videogame challenge, participants were provided with monetary rewards during the post-training session. The present study, like Goodie and Larkin's (1999) study, did not use monetary rewards for obtaining HR reduction at posttraining. The addition of rewards during the post-training phase may have provided an additional incentive to reduce HR in the Larkin et al. study. Regarding Sharpley's study, individuals not only learned to reduce HR using HR feedback, but were also provided with education and diaphragmatic breathing training. Therefore, both of these previous studies incorporated components other than HR feedback that may have contributed to the observed HR response reduction.

Blood pressure responses. Compared to the blood pressure levels observed during the pre-training assessment, SBP and DBP responses to tasks during the post-training assessments generally were equivalent to pre-training blood pressure levels. Because reduced HR responses generally failed to transfer to the short and long delay assessment sessions, it was not expected that blood pressure responses would decrease during those assessment phases. It is somewhat surprising that a few of the participants (i.e., 2 and 4) were able to reduce blood pressure reactions to some of the tasks at the short and long delay assessment phases without showing the expected reductions in HR response. This cardiovascular response pattern to the stressors might suggest that for these participants HR feedback training resulted in a broader physiological 
response affecting both cardiac and vascular responses to stress. Because this affect was not observed across all participants, however, HR feedback training did not always result in reduced blood pressure reactions. Additional research is warranted to examine what factors predict which participants will exhibit concomitant reductions in blood pressure response during HR feedback training. Previous studies (Goodie \& Larkin, 1999) have also demonstrated that blood pressure responses decreased following HR feedback training.

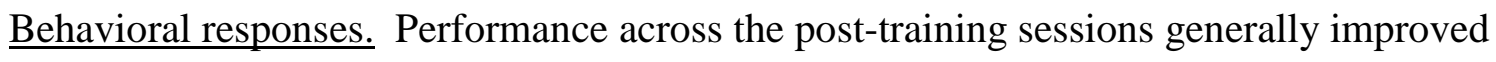
or remained stable when compared to the pre-training performance during all tasks. These data are consistent with the findings by Goodie and Larkin (1999) and Larkin et. al. (1990), which primarily examined performance during the videogame. The data from the present study suggested that attempts to reduce HR do not appear to interfere with performance during tasks that involve motor, cognitive, physical, or verbal responding.

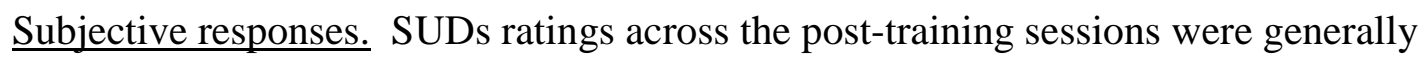
lower than those observed during the pre-training assessment. Systematic data regarding SUDs ratings have not been reported previously, so it is unclear how these data relate to previous studies. The SUDs data suggest that individuals became less emotionally aroused by the tasks, particularly after training. However, the data also suggest that individuals remained engaged in the tasks and found the tasks to be somewhat arousing throughout the entire study.

\section{$\underline{\text { Limitations and Future Directions }}$}

The findings of the present study support the hypothesis that individuals can learn to reduce their HR during HR feedback training in the presence of stressors requiring multiple behavioral response topographies. Individuals appear to learn these HR reduction skills without hindering their performance during the task, while experiencing consistent or increasing arousal. 
However, the results fail to support the hypothesis that individuals can demonstrate HR reduction without feedback present after extensive HR feedback training. Additionally, these data do not support the hypothesis that individuals can transfer their HR reduction skills to a task on which they have not received training. These data also suggest that the failure to reduce HR responses to tasks without HR feedback also results in limited reductions in blood pressure responses to those tasks.

There are several important limitations to the current study. First, there are the limitations that are inherent in a single subject design. Given the small number of participants in the present study it is unclear whether the observed changes in HR and blood pressure represent clinically important changes.

Secondly, the participants used in the present study were college students who were attempting to reduce their HR response to tasks for monetary gain. It is unknown whether these results generalize to other populations, particularly to older adults who are at greater risk for developing cardiovascular disease.

Third, the design of this study does not allow for comparisons between those who did and did not receive HR feedback. Therefore, it is unclear how HR, blood pressure, performance, and SUDs ratings would change over time without individuals attempting to reduce their HR using HR feedback training. Based upon previous data using control participants however, we can conclude that the observed reductions in HR were significantly better than what might have occurred through simple instructions to reduce HR.

Fourth, traditionally in single-subject design studies subjects are trained until they obtain the performance criterion. In this study, training was terminated after three 2-hour training sessions if the individuals had not lowered their HR response to the HR criterion. Although 
results from previous studies (i.e., Goodie \& Larkin, 2001; Larkin et al., 1989; Larkin et al., 1990; Larkin, Zayfert, Veltum, \& Abel, 1992) suggested that individuals would learn to reduce their HR responses to criterion within a single session, most participants in the current study failed to reduce their HR to the criterion after three training sessions. The difference between how the HR criterion was established between the present study and previous studies, as well as the highly variable baselines between sessions, may have contributed to the comparatively higher failure of participants to reach the HR criterion in the present study.

Fifth, individuals received monetary incentives to reduce their HR during the course of HR feedback training, but received no such incentives during pre-training and post-training assessments. Therefore, it is unclear whether the differences in monetary incentives between this study and previous research contributed to the reductions in HR responses during the training sessions and the relative lack of HR reductions observed during the post-training assessments. Fifth, the instability of baseline measures across sessions made it difficult to interpret the data. We chose to use the baseline measure observed during the pre-training assessment session as the initial baseline. However, the use of the more proximal baseline that occurred on the day of training or assessment session resulted in a more dramatic (i.e., below baseline levels) portrayal of HR response reductions. Therefore future studies may consider using a within session initial probe of the HR response to the training task, to obtain a more realistic goal for participants to obtain.

Sixth, the binary feedback (i.e., whether the person was above or below the HR criterion) provided in the present study, may not provide sufficient feedback to the participants about how they are progressing towards the HR criterion. Instead, providing participants with continuous visual feedback (e.g., graphically depicting HR values and the criterion level) about how close 
they are to the HR criterion, may increase their HR control.

Despite these limitations, the data from this study, and the study's design, demonstrate that individuals effectively learned to reduce their HR during HR feedback training, but failed to transfer those skills across time when HR feedback was no longer present and failed to transfer HR reduction skills to novel tasks. Overall, these results suggest that for the successful transfer of HR feedback training to daily tasks may require continuous training during encounters with daily stressors. Future studies should explore methods that may improve the transfer of HR feedback skills. Perhaps enhancing motivation during post-training sessions would improve the transfer of HR reduction skills. Additionally, future studies should examine the components of Sharpley's (1994) study, to determine whether HR feedback training is an important component to reducing HR in daily life settings. Alternatively, the effective component of Sharpley's study may have been instructions in diaphragmatic breathing. Continuing research on behavioral methods to reduce cardiovascular reactivity to stress may ultimately result in the development of prevention programs or cardiac rehabilitation programs designed to reduce the incidence of cardiovascular disease among high risk populations. 


\section{References}

Ahles, T. A., Blanchard, E. B., \& Leventhal, H. (1983). Cognitive control of pain: Attenuation to the sensory aspects of the cold pressor stimulus. Cognitive Therapy and Research, 7, 159-178.

Ainslie, G. W., \& Engel, B. T. (1974). Alternation of classically conditioned heart rate by operant reinforcement in monkeys. Journal of Comparative and Physiological Psychology, 87, 373-382.

Allan, R. \& Scheidt, S. (1996). Empirical basis for cardiac psychology. In R. Allen \& S. Scheidt (Eds.), Heart and mind: The practice of cardiac psychology (pp. 63-123). Washington, DC: American Psychological Association.

American Heart Association (1997). Heart and stroke statistical update. Dallas, TX: Author.

Andreassi, J. L. (1995). Psychophysiology: Human behavior and physiological responses. Hillsdale, NJ: Lawrence Erlbaum Associates.

Barefoot, J. C., Larsen, S., Von der Lieth, L., \& Schroll, M. (1995). Hostility incidence of acute myocardial infarction, and mortality in a sample of older Danish men and women. American Journal of Epidemiology, 142, 477-484.

Barlow, D. H. \& Hersen, M. (1984). Single case experimental designs: Strategies for studying behavior change. New York: Pergamon Press.

Barnett, P. A., Spence, D., Manuck, S. B., \& Jennings, J. R. (1997). Psychological stress and the progression of carotid artery disease. Journal of Hypertension, 15, 49-55.

Barnett, P. H., Hines, E. A., Schirger, A., \& Gage, R. P. (1963). Blood pressure and vascular reactivity to the cold pressor test. Journal of the American Medical Association, 183, 
$845-848$.

Bennett, D. H., Holmes, D. S., \& Frost, R. O. (1978). Effects of instructions, biofeedback, reward, and cognitive mediation on the control of heart rate and the application of that control in a stressful situation. Journal of Research in Personality, 12, 416-430.

Bentham, J. A., \& Glaros, A. G. (1982). Self control of stress-induced cardiovascular change using transit time feedback. Psychophysiology, 19, 502-505.

Blanchard, E. B., \& Young, L. D. (1972). Self-control of cardiac function: A promise as yet unfulfilled. Psychological Bulletin, 79, 145-163.

Booth-Kewley, S., \& Friedman, H. S. (1987). Psychological predictors of heart disease: A quantitative review. Psychological Bulletin, 101, 343-362.

Carroll, D., \& Evans, L. (1981). Effects of heart rate biofeedback and false biofeedback on reactions to stressful stimulation. Perceptual and Motor Skills, 53, 387-393.

Corse, C. D., Manuck, S. B., Cantwell, J. D., Giordani, B., \& Matthews, K. A. (1982). Coronary-prone behavior pattern and cardiovascular response in persons with and without coronary heart disease. Psychosomatic Medicine, 44, 449-459.

Davidoff, R., Schamroth, C. L., Goldman, A. P., Diamond, T. H., Cilliers, A. J., \& Myburgh, D. P. (1982). Postexercise blood pressure as a predictor of hypertension. Aviation, Space, and Environmental Medicine 53, 591-594.

Davig, J., Larkin, K. T., \& Goodie, J. L. (2001). Does cardiovascular reactivity to stress measured in the laboratory generalize to thesis and dissertation meetings among doctoral students? International Journal of Behavioral Medicine.

Edelstein, B. A. (1989). Generalization: Terminological, methodological, and conceptual 
issues. Behavior Therapy, 20, 311-324.

Engel, B. T., \& Chism, R. (1967). Operant conditioning of heart rate speeding, Psychophysiology, 3, 419-426.

Engel, B. T., \& Hansen, S. P. (1966). Operant conditioning of heart rate slowing. Psychophysiology, 3, 176-188.

Engel, B. T., \& Gottlieb, S. H. (1970). Differential operant conditioning of heart rate in the restrained monkey. Journal of Comparative and Physiological Psychology, 73, 217-225.

Ewart, C. K., Burnett, K. F., \& Taylor, C. B. (1983). Communication behaviors that affect blood pressure: An A-B-A-B analysis of marital interaction. Behavior Modification, 7 , $331-344$.

Faultisch, M.E., Williamson, D.A., McKenzie, S.J., Dutchman, E.G., Hutchinson, KM, \& Bouin, D.C. (1986). Temporal stability of psychophysiological responding: A comparative analysis of mental and physical stressors. International Journal of Neuroscience, 30, 65-72.

Frasure-Smith, N., Lesperance, F., \& Talajic, M. (1995). The impact of negative emotions on prognosis following myocardial infarction: Is it more than depression? Health Psychology, 14, 388-398.

Fredrikson, M. \& Matthews, K. A. (1990). Cardiovascular response to behavioral stress and hypertension: A meta-analytic review. Annals of Behavioral Medicine, 12, 30-39.

Goldstein, D. S., Ross, R. S., \& Brady, J. V. (1977). Biofeedback heart rate training during exercise. Biofeedback and Self-Regulation, 2, 107-125.

Goleman, D. J. \& Schwartz, G. E. (1976). Meditation as an intervention in stress reactivity. Journal of Consulting and Clinical Psychology, 44, 456-466. 
Goodie, J. L., \& Larkin, K. T. (1999). Using impedance cardiography to examine changes in hemodynamic parameters during heart rate biofeedback. Unpublished master's thesis, West Virginia University, Morgantown, West Virginia.

Green, K. D., Webster, J., Beiman, I., Rosmarin, D., \& Holliday, P. (1981). Progressive and self-induced relaxation training: Their relative effects on participantive and autonomic arousal to fearful stimuli. Journal of Clinical Psychology, 37, 309-315.

Grimm, L., \& Kanfer, F. H. (1976). Tolerance of aversive stimulation. Behavior Therapy, 7, 593-601.

Hastrup, Light, \& Obrist (1982). Parental hypertension and cardiovascular response to stress in healthy young adults. Psychophysiology, 19, 615-622.

Hatch, J. P. (1980). The effects of operant reinforcement schedules on the modification of human heart rate. Psychophysiology, 17, 559-567.

Hollenberg, N. K., Williams, G. H., \& Adams, D. F. (1981). Essential hypertension: abnormal renal, vascular, and endocrine responses to a mild psychological stimulus. Hypertension, 3, 11-17.

Houston, B. K., \& Holmes, D. (1974). Effect of avoidant thinking and reappraisal for coping with threat involving temporary uncertainty. Journal of Personality and Social Psychology, 3, 382-388.

Jenkins, C. D. (1988). Epidemiology of cardiovascular diseases. Journal of Consulting and Clinical Psychology, 56, 324-332.

Jorgenson, R. S., Houston, B. K., \& Zurawski, R. M. (1981). Anxiety management training in the treatment of essential hypertension. Behavior Research and Therapy, 19, 467-474.

Keys, A., Taylor, H. L., Blackburn, H., Brozek, J., Anderson, J. T., \& Simonson, E. 
(1971). Mortality and coronary heart disease among men studied for 23 years. Archives of Internal Medicine, 128, 201-214.

Kirsch, I., \& Henry, D. (1979). Self-desensitization and meditation in the reduction of public speaking anxiety. Journal of Consulting and Clinical Psychology, 47, 536-541.

Krantz, D. S., \& Manuck, S. B. (1984). Acute psychophysiological reactivity and risk for cardiovascular disease: A review and methodologic critique. Psychological Bulletin, 96, 435464.

Lacey, J. I., Bateman, D. E., \& Van Lehn, R. (1953). Autonomic response specificity: An experimental study. Psychosomatic Medicine, 15, 8-21.

Larkin, K. T., Manuck, S. B. \& Kasprowicz, A. L. (1989). Heart rate feedback-assisted reduction in cardiovascular reactivity to a videogame challenge. The Psychological Record, 39, $365-371$.

Larkin, K. T., Manuck, S. B., \& Kasprowicz, A. L. (1990). The effect of feedbackassisted reduction in heart rate reactivity on videogame performance. Biofeedback and SelfRegulation, 15, 285-303.

Larkin, K. T., Zayfert, C., Veltum, L. G., \& Abel, J. L. (1992). Effects of feedback and contingent reinforcement in reducing heart rate response to stress. Journal of Psychophysiology, 6, 119-130.

Larkin, K. T., Zayfert, C., Abel, J. L., \& Veltum, L. G. (1992). Reducing heart rate reactivity to stress with feedback. Behavior Modification, 16, 118-131.

Lehrer, P. M. (1978). Psychophysiological effects of progressive relaxation in anxiety neurotic patients and of progressive relaxation and alpha feedback in non-patients. Journal of Consulting and Clinical Psychology, 46, 389-404. 
Lejuez, C. W., Zvolensky, M. J., \& Eifert, G. H. (1999). Using a single-subject design to assess the development of anxiety in humans. Journal of Behavior Therapy and Experimental Psychiatry, 30, 15-20.

Malmo, R. B., Shagass, C., \& Davis, F. H. (1950). Symptom specificity and bodily reactions during psychiatric interview. Psychosomatic Medicine, 12, 362-376.

Manuck, S. B. (1994). Cardiovascular reactivity in cardiovascular disease: "Once more unto the breach.” International Journal of Behavioral Medicine, 1, 4-31.

Manuck, S. B., Kaplan, J. R., Adams, M. R., \& Clarkson, T. B. (1989). Behaviorally elicited heart rate reactivity and atherosclerosis in female cynomolgus monkeys (Mascaca fascisularis). Psychosomatic Medicine, 51, 306-318.

Manuck, S. B., Kaplan, J. R., \& Clarkson, T. B. (1983). Social instability and coronary artery atherosclerosis in cynomolgus monkeys. Neuroscience and Biobehavioral Reviews, 7 , 485-491.

Manuck, S. B., Kasprowicz, A. L., Monroe, S. B., Larkin, K. T., \& Kaplan, J. R. (1989). Psychophysiologic reactivity as a dimension of individual differences. In N. Schneiderman, S.B. Weiss, f \& P. Kaufmann (Eds.), Handbook of methods and measurements in cardiovascular behavioral medicine (pp. 365-382). New York: Plenum.

Manuck, S. B., Levenson, R. W., Hinrichsen, J. J., \& Gryll, S. L. (1975). Role of feedback in voluntary control of heart rate. Perceptual and Motor Skills, 40, 747-752.

Matthews, K. A., Manuck, S. B., \& Saab, P.G. (1986). Cardiovascular responses of adolescents during a naturally-occurring stressor and their behavioral and psychophysiological predictors. Psychophysiology, 23, 198-209.

Matthews, C. E., Pate, R. R., Jackson, K. L., Ward, D. S., Macera, C. A., Kohl, H. W., \& Blair, S. N. (1998). Exaggerated blood pressure response to dynamic exercise and risk for future 
hypertension. Journal of Clinical Epidemiology, 51, 29-35.

Menkes, M. S., Matthews, K. A., Krantz, D. S., Lundberg, U., Mead, L. A., Qaqish, B., Liang, K. Y., Thomas, C. B., Pearson, T. A. (1989). Cardiovascular reactivity to the cold pressor test as a predictor of hypertension. Hypertension, 14, 524-530.

Moos, R. H., \& Engel, B. T. (1962). Psychophysiological reactions in hypertensive and arthritic patients. Journal of Psychosomatic Research, 6, 227-241.

Orth-Gomer, K. (1994). International epidemiological evidence for a relationship between social support and cardiovascular disease. In S. A. Shumaker \& S. M. Czajkowski (Eds.), Social support and cardiovascular disease (pp. 97-117). New York: Plenum.

Perski, A., \& Engel, B. T. (1980). The role of behavioral conditioning in the cardiovascular adjustment to exercise. Biofeedback and Self-Regulation, 5, 91-104.

Ruberman, W., Weinblatt, E., Goldberg, J., \& Chaudhary, B. S. (1984). Psychosocial influences on mortality after myocardial infarction. New England Journal of Medicine, 311, 552559.

Sawada, Y., \& Steptoe, A. (1988). The effects of brief meditation training on cardiovascular stress responses. Journal of Psychophysiology, 2, 249-257.

Sharpley, C. F. (1989). Biofeedback training versus simple instructions to reduce heart rate reactivity to a psychological stressor. Journal of Behavioral Medicine, 12, 435-447.

Sirota, A. D., Schwartz, G. E., \& Shapiro, D. (1974). Voluntary control of human heart rate: Effect on reaction to aversive stimulation. Journal of Abnormal Psychology, 83, 261-267.

Smith, T. W., \& Leon, A. (1992). Coronary heart disease. Champaign, IL: Research Press.

Turner, J.R., Carroll, D., Dean, S., \& Harris, M.G. (1987). Heart rate reactions to 
standard laboratory challenges and a naturalistic stressor. International Journal of

Psychophysiology, 5, 151-152.

Turner, J. R., Girdler, S. S., Sherwood, A., \& Light, K. C. (1990). Cardiovascular responses to behavioral stressors: Laboratory-field generalization and inter-task consistency. Journal of Psychosomatic Research, 34, 581-589.

Weems, C. F. (1998). The evaluation of heart rate biofeedback using a multi-element design. Journal of Behavior Therapy and Experimental Psychiatry, 29, 157-162. 


\section{Footnotes}

${ }^{1}$ Demonstrating control skills (e.g., HR reduction) in the presence of one stressor when trained in the presence of another stressor is more appropriately referred to as "transfer of training," rather than the more commonly used term of "generalization" (Edelstein, 1989). 
Appendix A

\section{SUDS}

Please rate your level of stress/arousal during the previous rest period/task.

0

$10 \quad 20 \quad 30 \quad 40$

40

50

60

70

80

90

100

Most

Most

Relaxed

Stressed 


\section{Appendix B}

\section{PRE-TRAINING}

\begin{tabular}{|l|}
\hline Participants attend 2-hour session \\
10 min habituation \\
Videogame presented until HR habituation \\
Mental arithmetic presented until HR habituation \\
Hand grip presented until HR habituation \\
Speech task presented once \\
\hline
\end{tabular}

6 individuals with highest HR response selected to continue in studv.

\begin{tabular}{|l|l} 
Videogame training & Target \\
10 min habituation \\
resting HR measured \\
videogame repeatedly presented
\end{tabular}

\section{Target HR met.}

\section{VIDEOGAME TRAINING}

Videogame training

10 min habituation

resting HR measured

videogame repeatedly presented

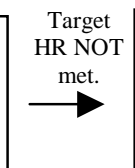

Videogame training 10 min habituation resting HR measured videogame repeatedly presented

IMMEDIATE POST TRAINING

Target HR met.

Target HR met or time expires.

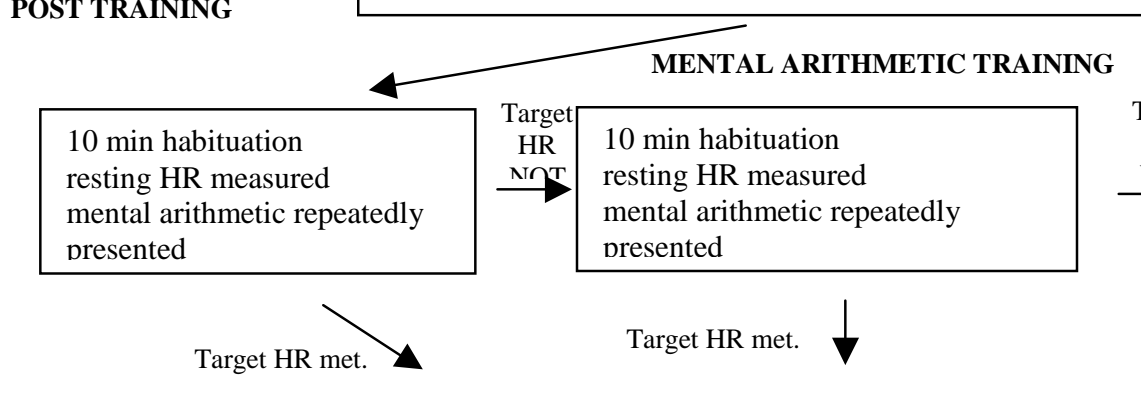

\section{IMMEDIATE POST TRAINING}

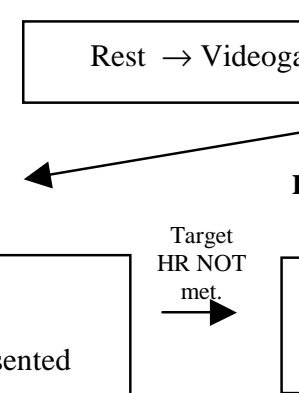

10 min habituation resting HR measured hand grip repeatedly presented

\section{HAND GRIP TRAINING}

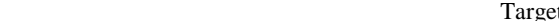

10 min habituation

resting HR measured

hand grip repeatedly presented

Target HR met.

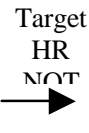

10 min habituation resting HR measured mental arithmetic repeatedly presented

Target HR met or time exnires.

Target HR met.

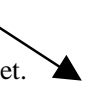

IMMEDIATE POST TRAINING

$$
\text { Rest } \rightarrow \text { Videogame } \rightarrow \text { Rest } \rightarrow \text { Mental Arithmetic } \rightarrow \text { Rest } \rightarrow \text { Handgrip }
$$

SHORT DELAY POST TRAINING

$$
\text { 1-2 davs }
$$

Rest $\rightarrow$ Videogame $\rightarrow$ Rest $\rightarrow$ Mental Arithmetic $\rightarrow$ Rest $\rightarrow$ Handgrip $\rightarrow$ Rest $\rightarrow$ Speech Task

LONG DELAY POST TRAINING

\section{1-2 weeks}

Rest $\rightarrow$ Videogame $\rightarrow$ Rest $\rightarrow$ Mental Arithmetic $\rightarrow$ Rest $\rightarrow$ Handgrip $\rightarrow$ Rest $\rightarrow$ Speech Task 


\section{Appendix C}

\section{Consent Form}

\section{Heart Rate Feedback Training During Laboratory Tasks}

Introduction: I, which has been explained to me by have been asked to participate in this research study, Jeffrey Goodie and Dr. Kevin Larkin at West Virginia University for partial fulfillment of the requirements for Jeffrey Goodie's dissertation.

Purpose of the Study: I understand that the purpose of this research project is to examine how skills learned during heart rate transfer between tasks.

Description of Procedures: I understand that there are two phases to this study, Phase I and Phase II. I further understand that my participation in this study will involve the measurement of my heart rate using a finger heart rate monitor and three spot electrodes will also be attached to my torso. Blood pressure will also be measured using a blood pressure cuff wrapped around my arm.

Phase I: During Phase I of the study I will be requested to participate in an approximately 2 hour session. During the session, I will be asked to sit quietly for a baseline period. I will then be asked to participate in a videogame, mental arithmetic, hand grip, and speech task. The videogame, mental arithmetic, and hand grip tasks will be presented multiple times as my heart rate and blood pressure are monitored. The exact number of times each task is presented will depend on my cardiovascular responses. I will be asked to complete 1 questionnaire. I understand that I can review this questionnaire before signing this consent agreement and that I do not have to answer all questions. Approximately 20 subjects will be entered in this phase of the study. I understand that based on their cardiovascular responses to the tasks, 6 individuals will be selected to participate in Phase II of this study and that my participation in Phase I of this study does not guarantee that I will be selected for the second phase.

Phase II: During Phase II of the study I will be asked to participate in at approximately 5 separate sessions over a 2 week period. During the five sessions I will be asked to reduce my heart rate while the videogame, mental arithmetic, hand grip, or speech task is presented. The sessions will occur across contiguous days, except for the final session, which will occur approximately 1 week after the previous session. I understand that based on my ability to reduce my heart rate, additional sessions may be needed for additional practice with heart rate reduction. Each session will last between 1-2 hours.
Date 
Risks and Discomforts: My participation in this study is not expected to produce a risk to my health or well-being. I understand that some discomfort and irritation may arise during the placement and removal of the strip and spot electrodes. I may also experience some discomfort in my arm during the repeated blood pressure measures. However, the procedures involved in this study may have risks that are unforeseeable. In the unlikely event that I incur an injury as a result of my participation in this research, I understand that voluntary compensation or costs for medical treatment will not be provided by West Virginia University for research-related injuries. I am aware that the experimental procedures will be terminated if at any time during the laboratory sessions, my blood pressure is found to be above $200 \mathrm{mmHg}$ (systolic) and/ 120 $\mathrm{mmHg}$ (diastolic). In the event of such termination of the experiment, I understand that I will receive a proportion (reflecting the extent of my participation) of the class credit and lottery entries described below.

\section{Compensatory Considerations:}

Phase I: I understand that I will receive extra credit points toward my psychology course grade in return for my participation in Phase I of this study. I also understand that other methods of obtaining extra credit are available in my class. No monetary compensation will be provided for participation in Phase I of this study.

Phase II: If I am selected for Phase II of this study, I understand that based on my performance during the videogame, mental arithmetic, hand grip, and speech tasks I will earn a cash reward. However, if my performance does not meet the criteria established by the researcher during a given task, I understand that I may not qualify for a cash reward. I am also aware that, regardless of my performance during the tasks, I will be paid $\$ 10$ for my complete participation in the second phase. Additionally, I will receive extra credit points toward my psychology course grade in return for my participation in Phase II of this study. I also understand that other methods of obtaining extra credit are available in my class.

Benefits: My participation in this study may lead to an increased understanding of the benefits of heart rate feedback training.

Contact Persons: For more information about this research and about research-related risks or injury, I should contact Jeffrey Goodie at 293-2001 ext. 859 or Dr. Kevin T. Larkin at 293-2001 ext. 668. For information regarding my rights as a research subject, I may contact the Executive Secretary of the Institutional Review Board for the Protection of Human Subjects at 293-7073.

Confidentiality: I understand that any information about me obtained as a result of my participation in this research will be kept as confidential as legally possible. I understand that my research records, just like hospital records, may be subpoenaed by court order or may be inspected by federal regulatory agencies. In any publications result from this research, neither my name nor any information from which I might be identified, will be published without my consent.

Initials

Date 
Voluntary Consent: Participation in this study is voluntary. Refusal to participate or withdrawal will involve no penalty or loss of benefits and will not affect my grades or class standing. I have been given the opportunity to ask questions about the research, and I have received answers concerning areas I did not understand. In the event new information becomes available that may affect my willingness to continue to participate in this study, this information will be given to me so I may make an informed decision about my participation.

Upon signing this form, I will receive a copy.

I willingly consent to participate in this study.

Signature of Subject or Subject's Representative

Signature of Investigator or Investigator's Representative
Date

Date 


\section{Appedix D}

\section{Demographics Form}

Subject ID:

Date:

Arrival time:

1. Age

2. Race

3. Height

4. Weight

5. Hip

$$
\text { Waist }
$$

Ratio

6. What is your current relationship status?

Not dating Dating same person: < 1 mnth 1-6 mnths 6-12 mnths > 12 mnths

7. Years of education? $($ high school $=12)$

8. Do you smoke? Y N

How much (pks/day)

How long? (yrs)

When was the last time? (hours)

9. Do you consume alcohol? Y N

What?

How much? (drinks/month)

For how long? (yrs)

10. Do you consume caffeine? (e.g., soda, coffee, tea, etc...) Y N

How much?

Last time? (cups/day) (hours)

11. Do you exercise regularly? $\mathrm{Y} N$

How much? (min/wk)

For how long? (yrs)

When was the last time? (days)

12. Do you use any recreational or street drugs?

(e.g. marijuana, crack, cocaine, heroin)

What?

How frequently? (days per month)

How much per episode? 
Last time? (days ago)

13. Are you taking any medications? $\mathrm{Y} N$ Drug Name

Purpose

14. Do you consume a special diet (e.g., salt restriction, low fat, etc...)? Y N For how long? (yrs)

15. Have you been diagnosed with any chronic medical conditions? $\mathrm{Y} N$ Describe:

16. Have you ever been diagnosed with a cardiovascular problem? Y N (e.g., hypertension, mitral valve prolapse) Describe:

17. Is there a family history of any cardiovascular disease in your family? $\mathrm{Y} \mathrm{N}$ Describe:

18. Has anyone in your family been diagnosed with hypertension? $\mathrm{Y} N$ Describe: 
Appendix E

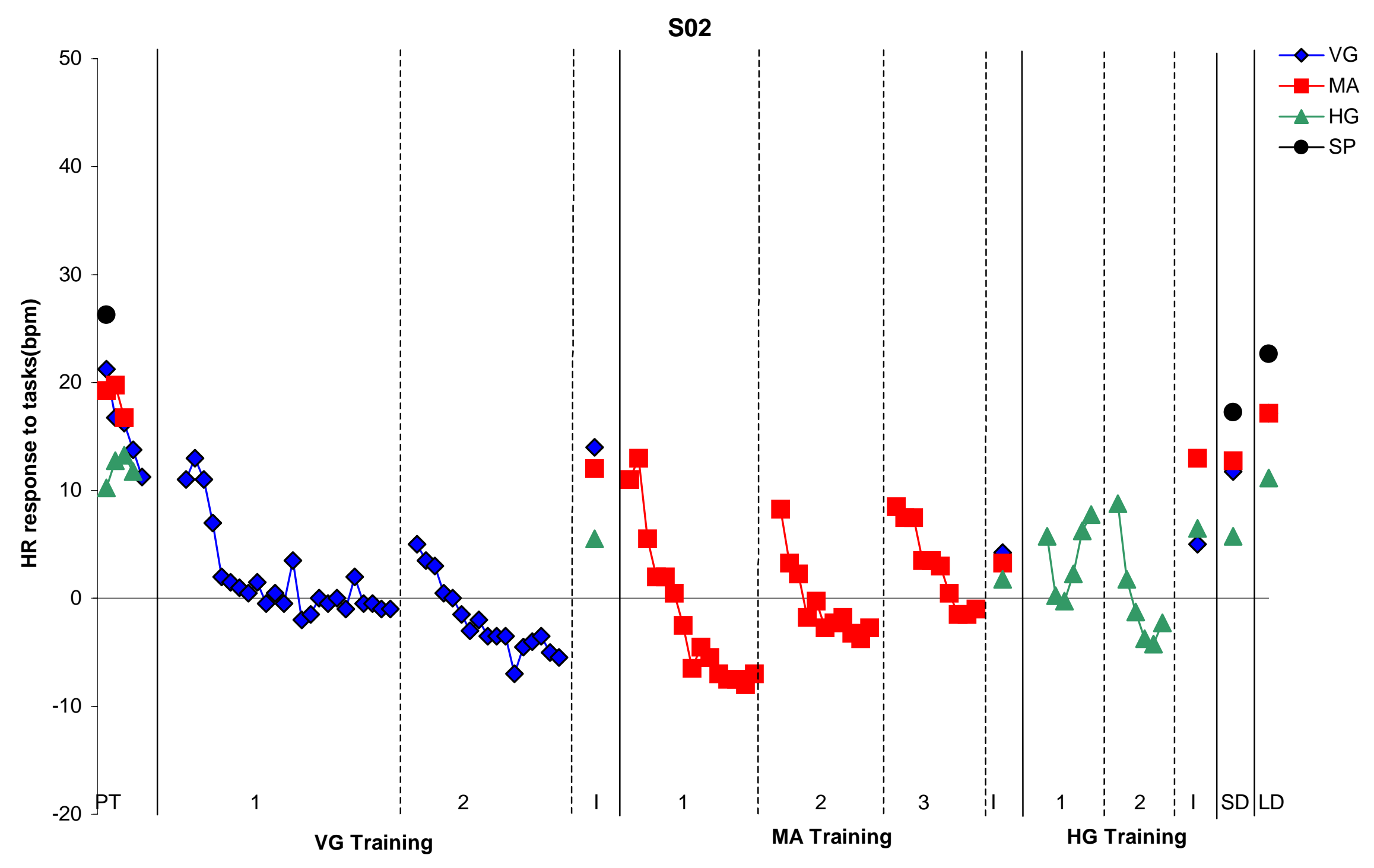




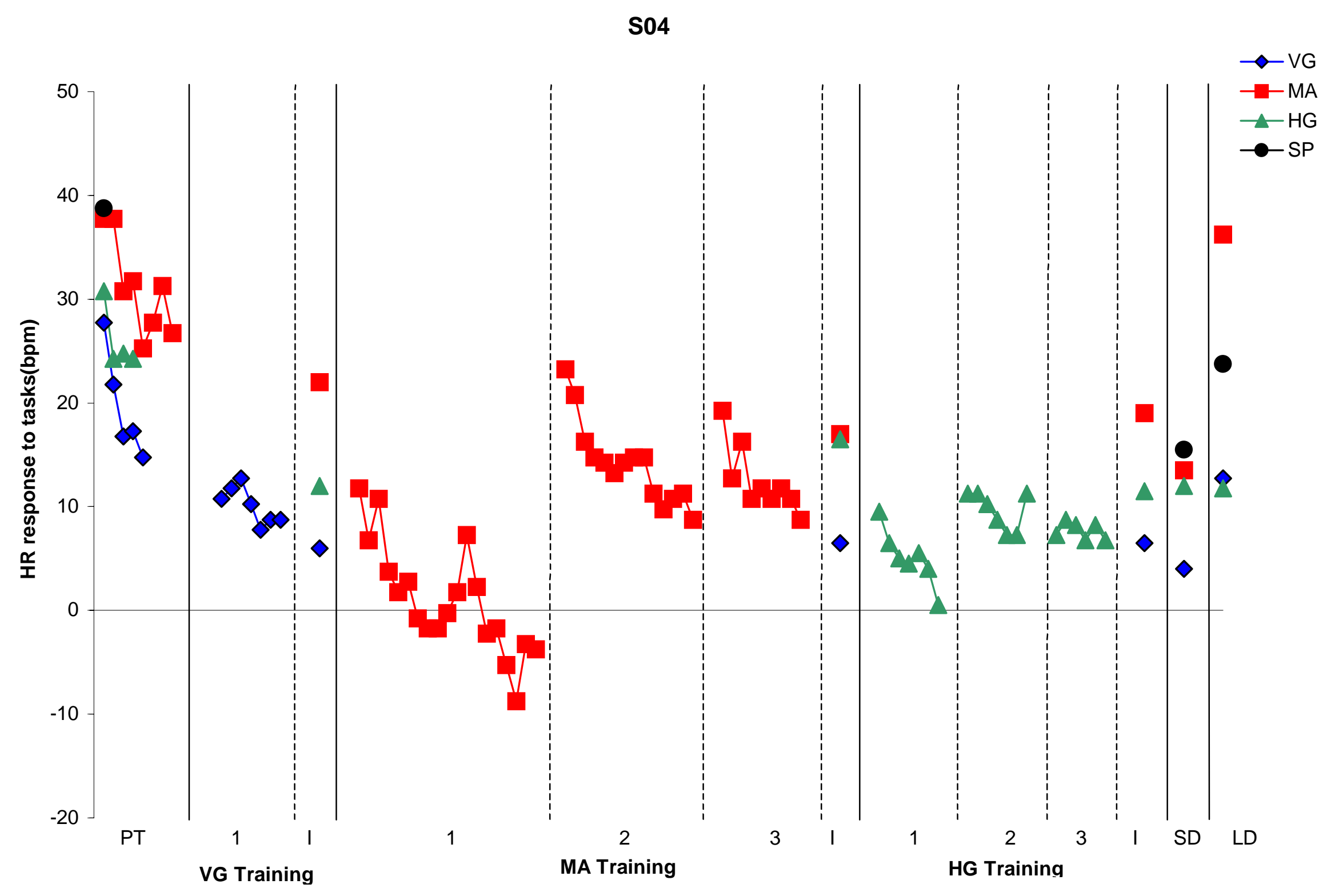




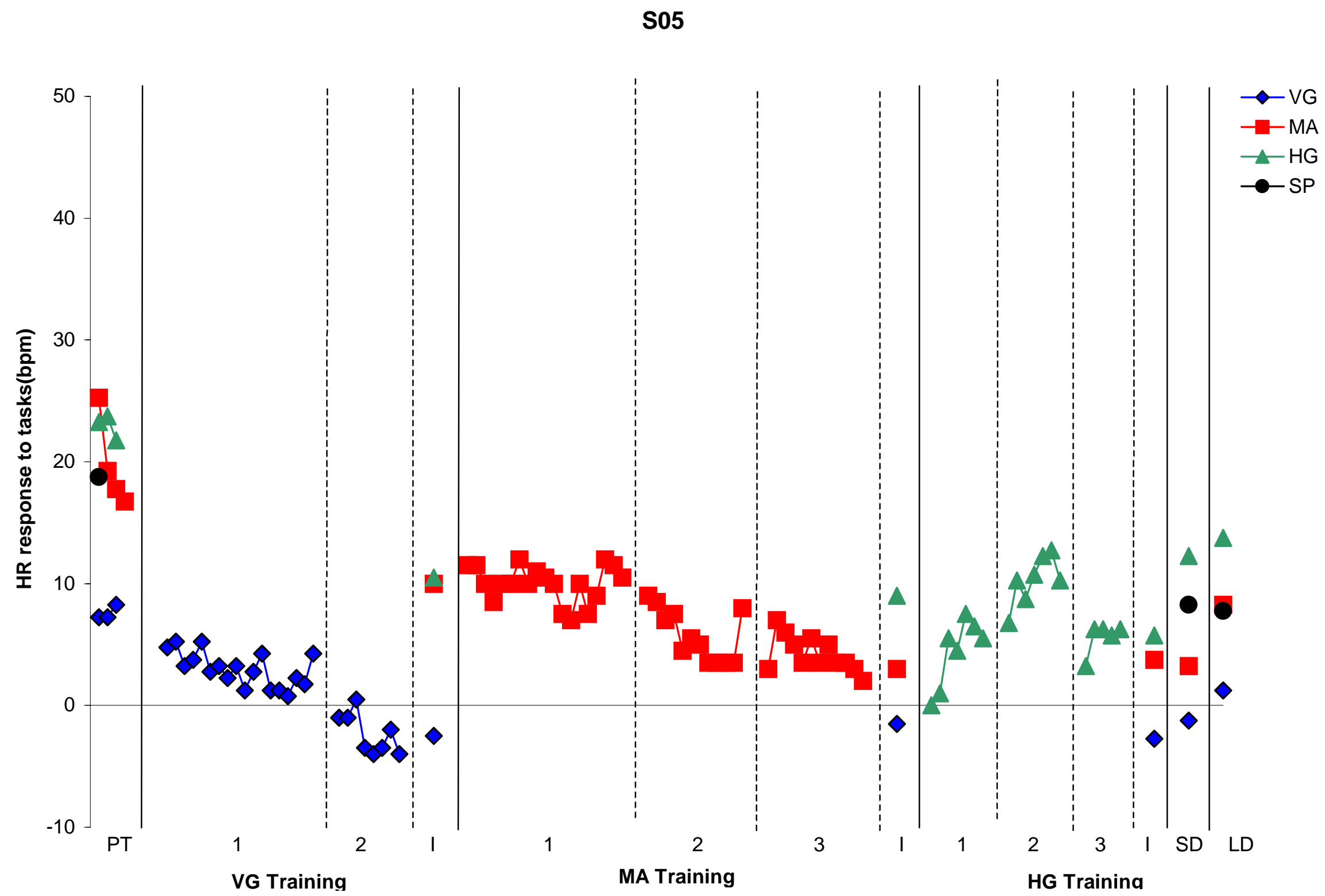


S10

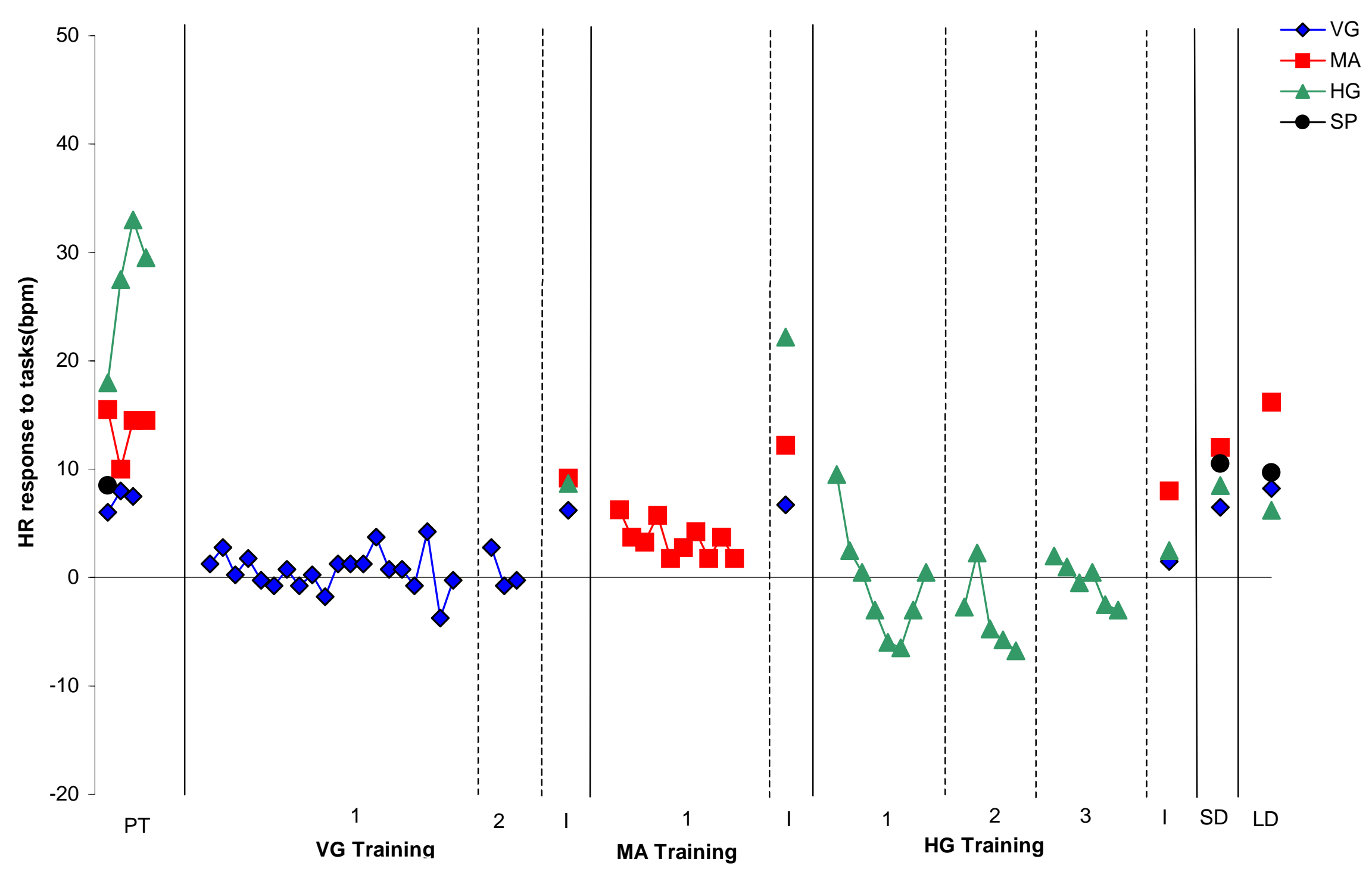




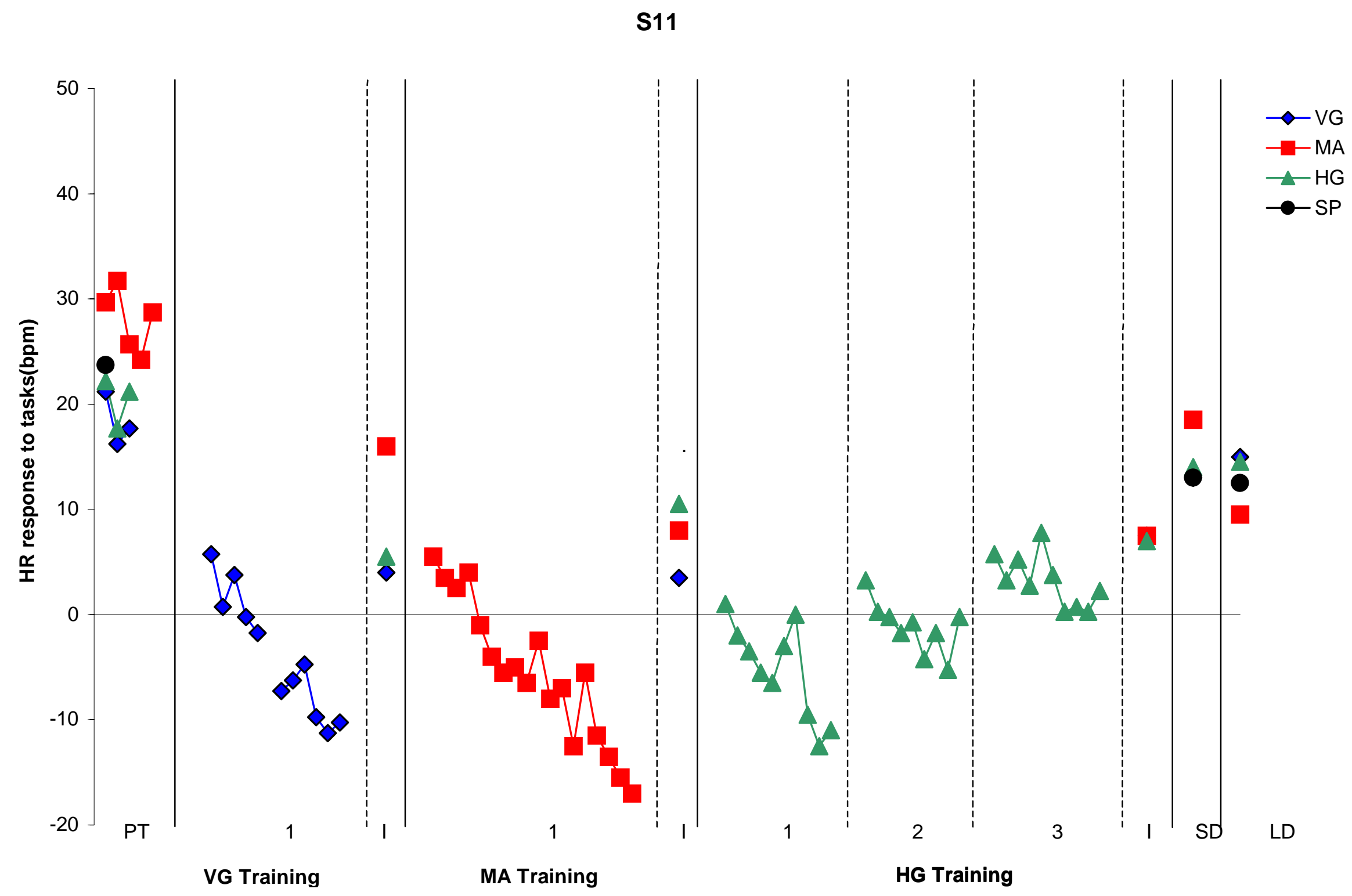




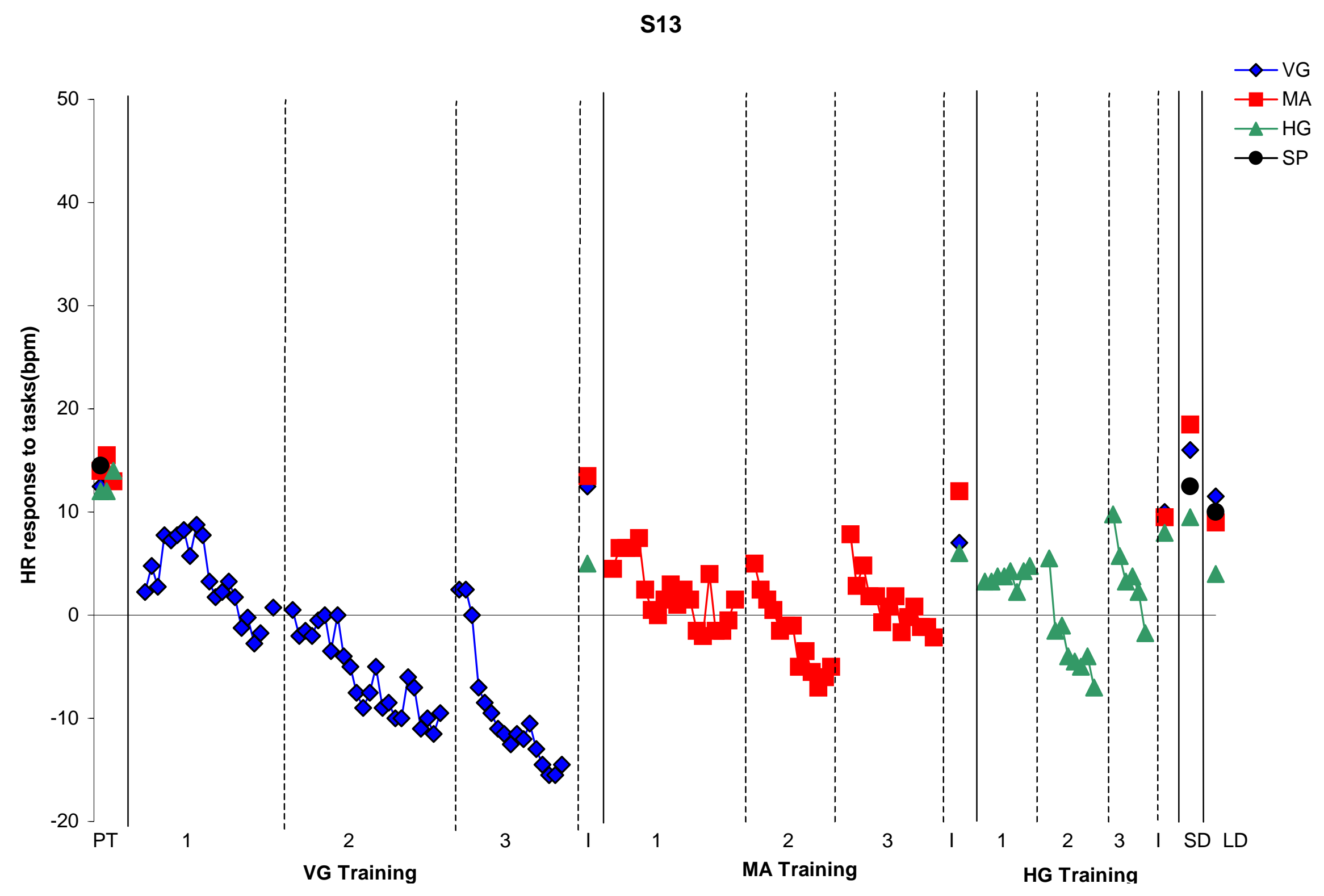


Table 1. Demographics of study participants.

\begin{tabular}{|c|c|c|c|c|c|c|c|c|c|c|}
\hline Subject \# & Sex & Age & Race & BMI & $\begin{array}{l}\text { Smoke } \\
(\mathrm{Y} / \mathrm{N})\end{array}$ & $\begin{array}{l}\text { Alcohol } \\
(\mathrm{Y} / \mathrm{N})\end{array}$ & $\begin{array}{c}\text { Caffeine } \\
\text { Use }(\mathrm{Y} / \mathrm{N})\end{array}$ & $\begin{array}{c}\text { Family Hx } \\
\text { of CVD }\end{array}$ & $\begin{array}{l}\text { Family Hx of } \\
\text { Hypertension }\end{array}$ & $\begin{array}{l}\text { Relaxation } \\
\text { technique }\end{array}$ \\
\hline 1 & female & 20 & caucasian & 37.0 & yes & no & yes & no & yes & no \\
\hline 2 & female & 18 & caucasian & 26.6 & no & no & yes & yes & no & no \\
\hline 3 & female & 20 & caucasian & 28.2 & no & no & yes & yes & yes & no \\
\hline 4 & male & 21 & caucasian & 21.3 & no & yes & yes & no & yes & yes \\
\hline 5 & male & 19 & caucasian & 25.1 & no & yes & yes & no & no & no \\
\hline 6 & male & 19 & caucasian & 21.9 & no & yes & yes & no & no & yes \\
\hline 7 & female & 35 & caucasian & 30.7 & yes & yes & yes & no & no & no \\
\hline 8 & male & 19 & caucasian & 25.1 & yes & yes & yes & no & no & no \\
\hline 9 & female & 18 & black & 19.3 & yes & yes & no & yes & yes & no \\
\hline 10 & male & 18 & caucasian & 23.5 & no & yes & yes & yes & no & no \\
\hline 11 & female & 18 & caucasian & 20.1 & no & no & yes & no & no & yes \\
\hline 12 & male & 18 & caucasian & 31.3 & no & no & yes & yes & yes & no \\
\hline 13 & female & 27 & black & 21.0 & no & yes & yes & yes & yes & no \\
\hline 14 & male & 19 & caucasian & 19.8 & no & no & yes & no & yes & no \\
\hline
\end{tabular}

Note: Participants that are bolded were selected for heart rate feeback training. 


\section{Figure 1.}

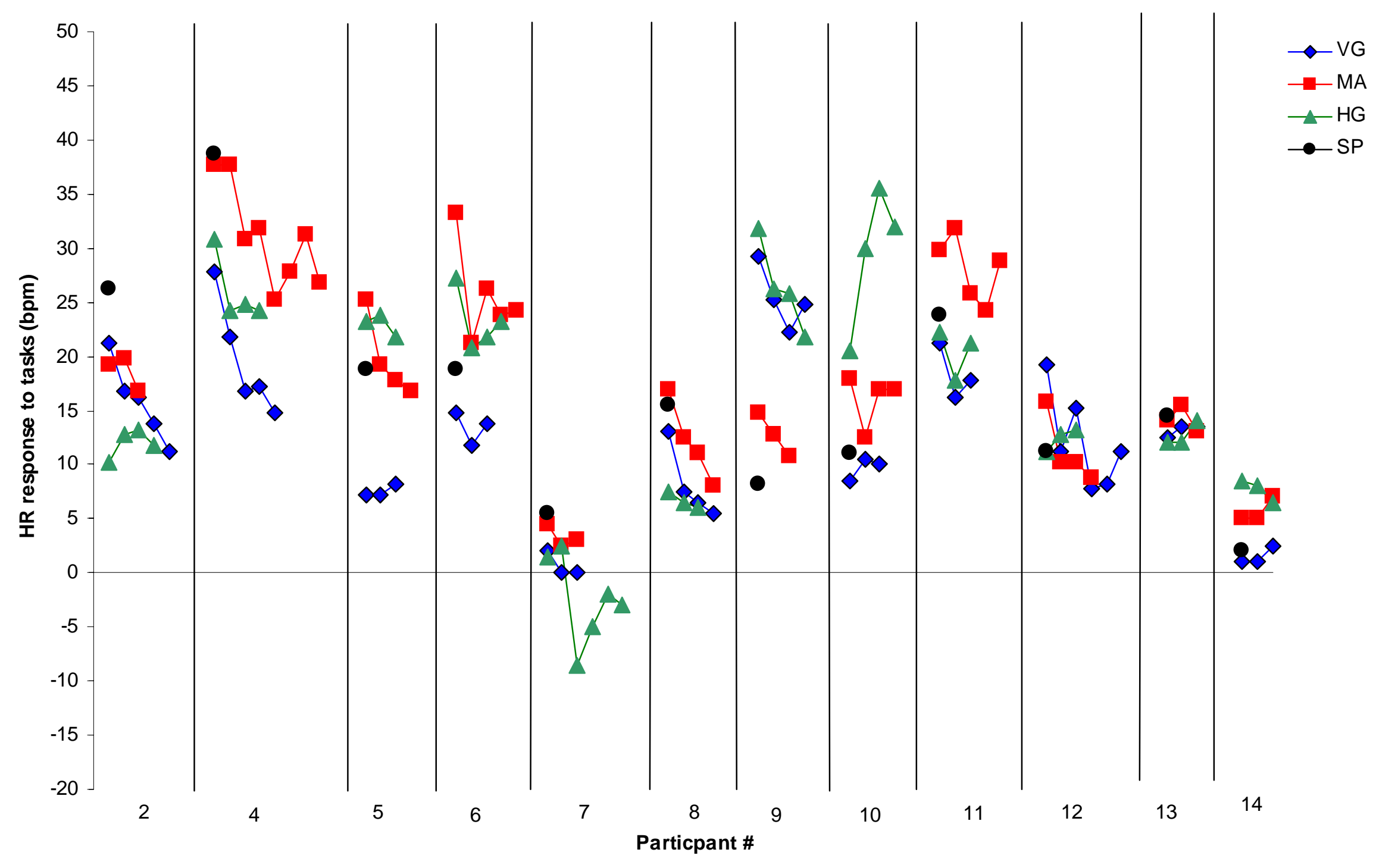


Figure 2.

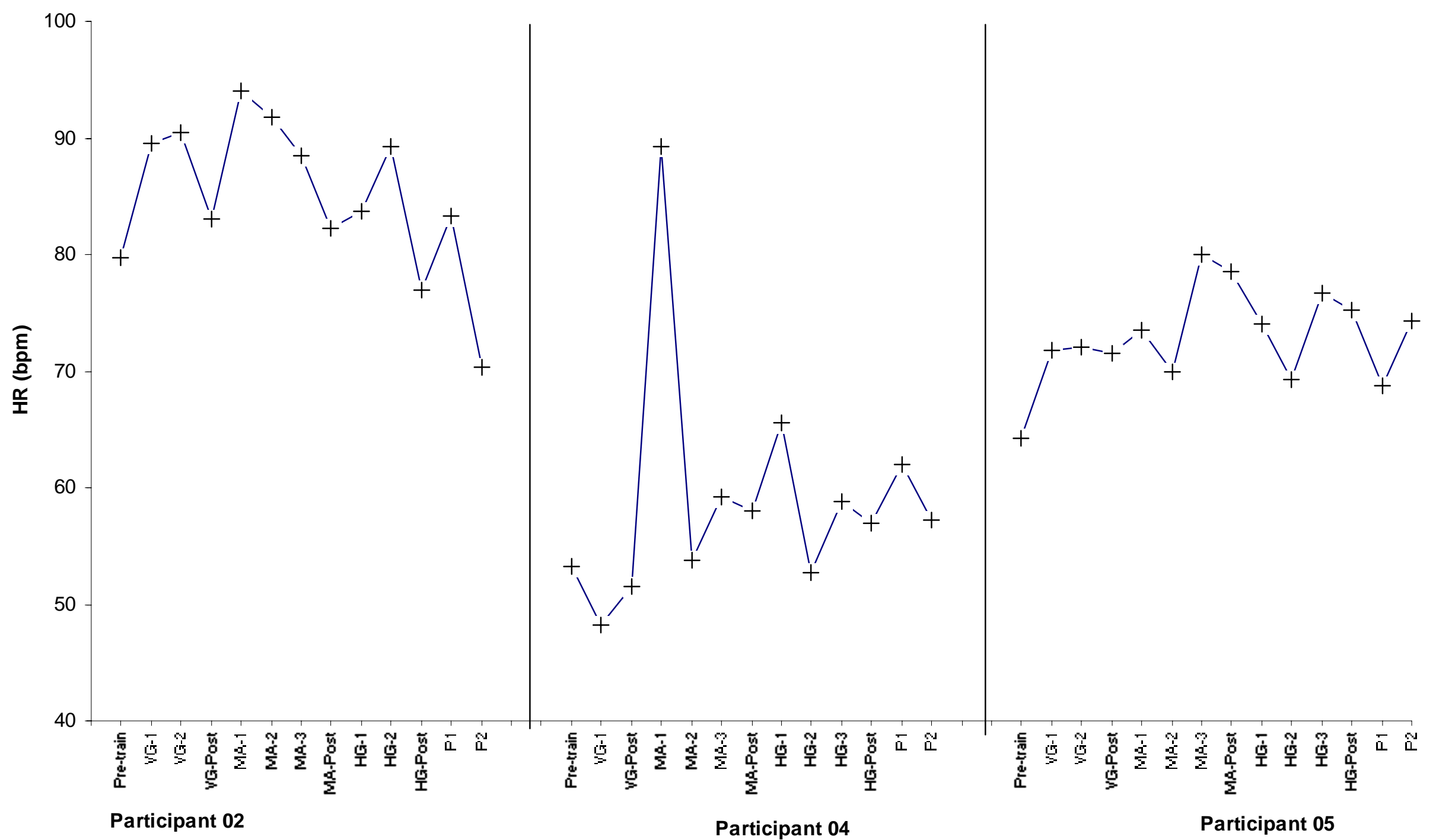


Figure 3.

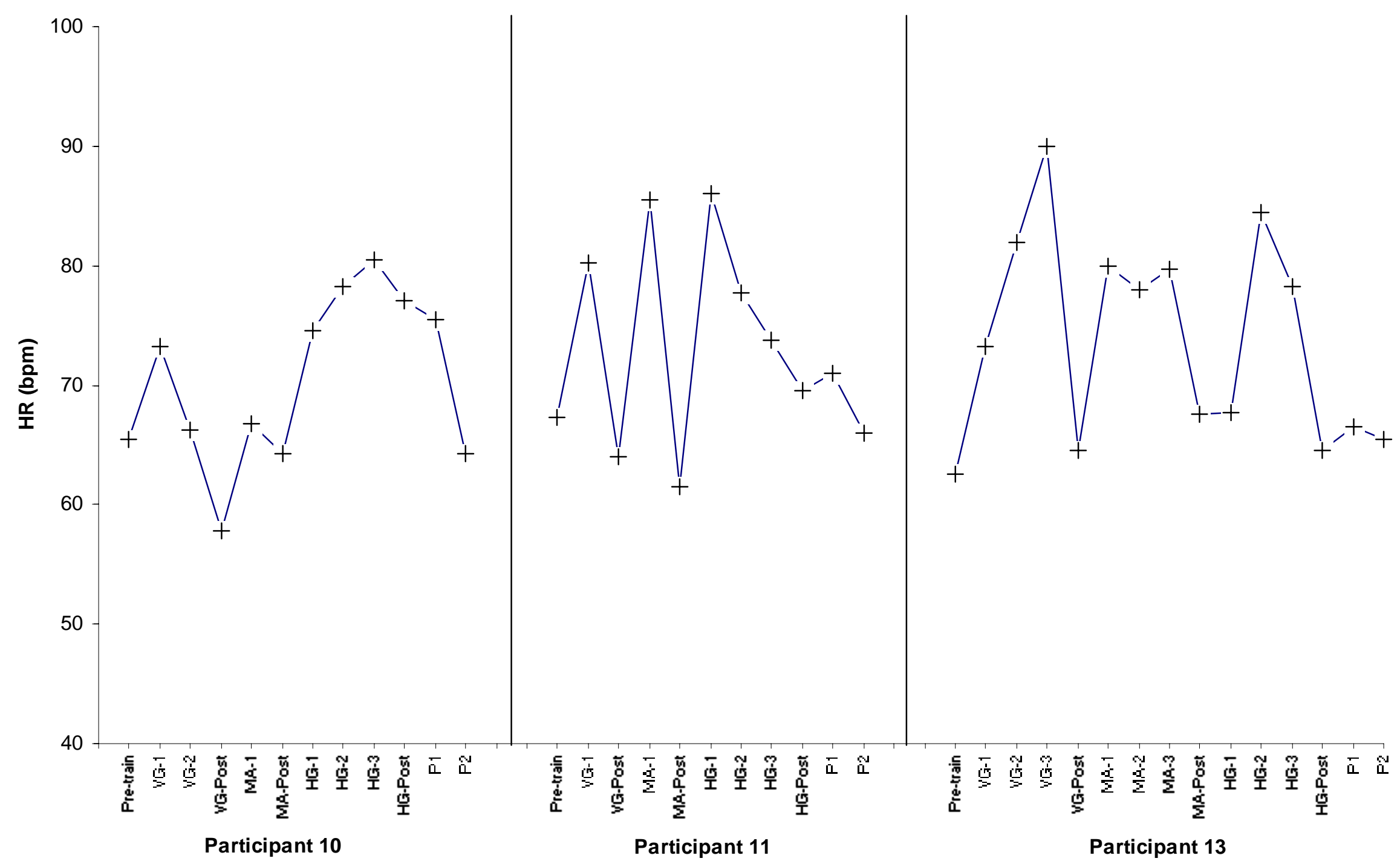


Figure 4.

S02

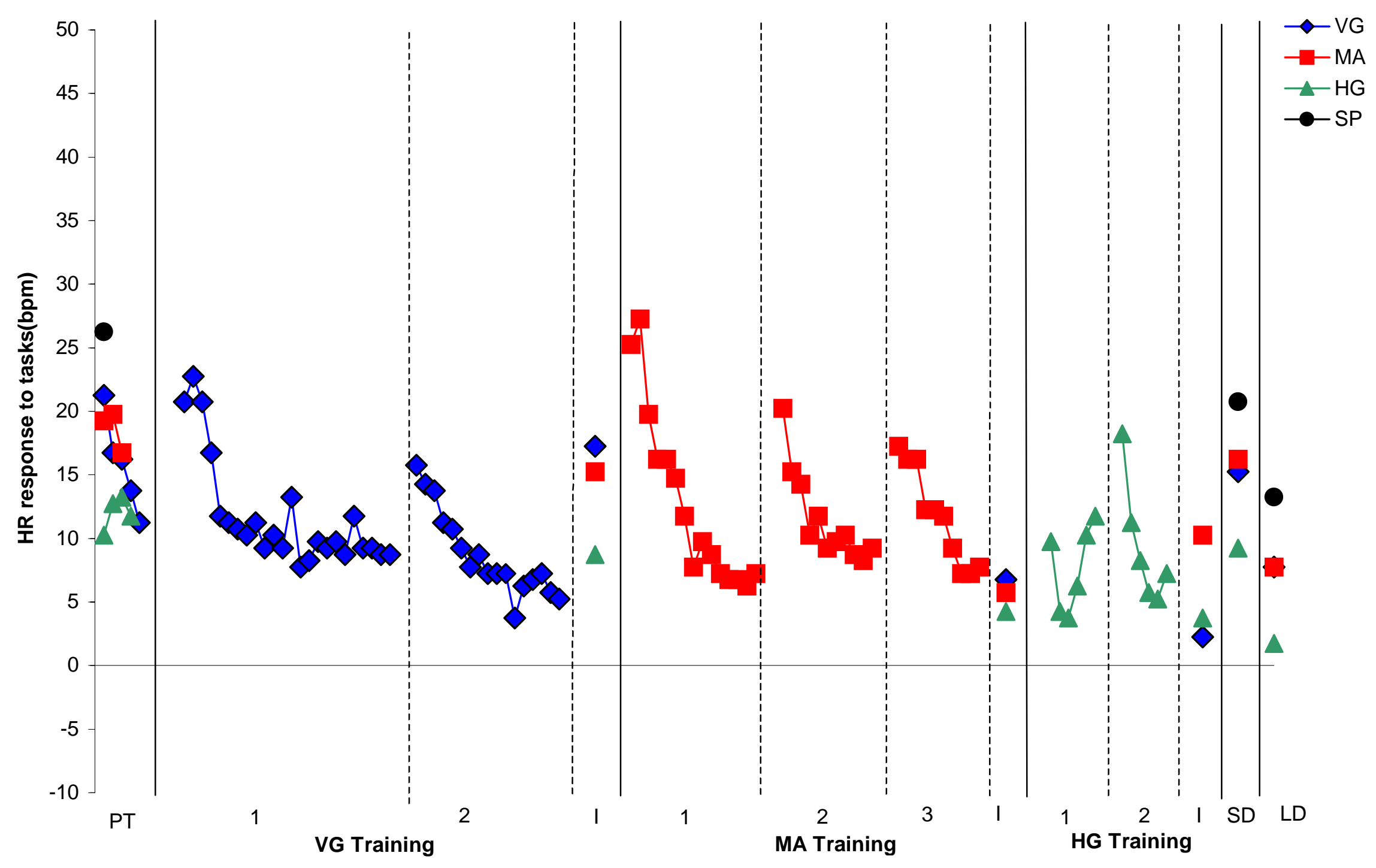




\section{Figure 5.}

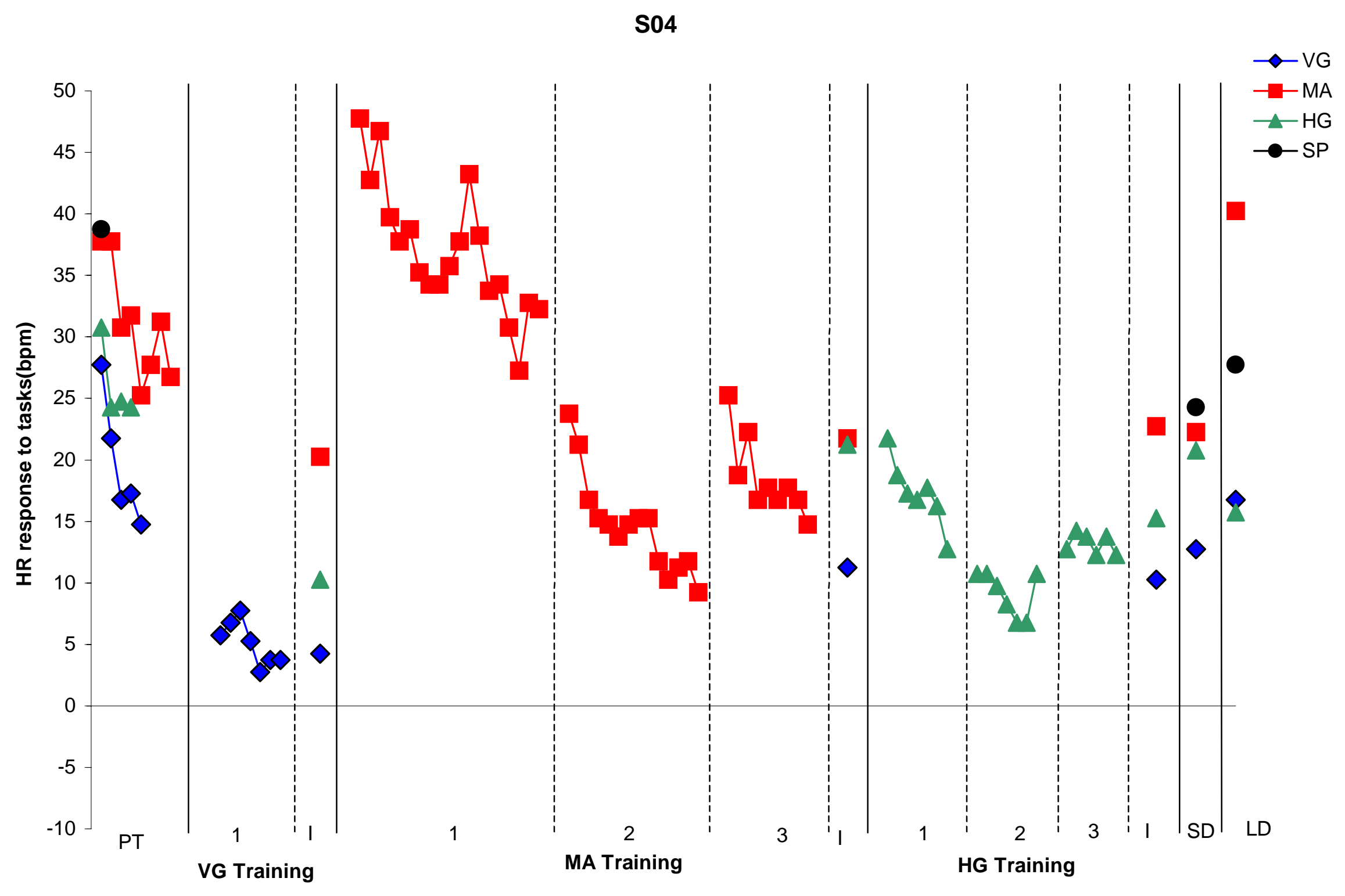




\section{Figure 6.}

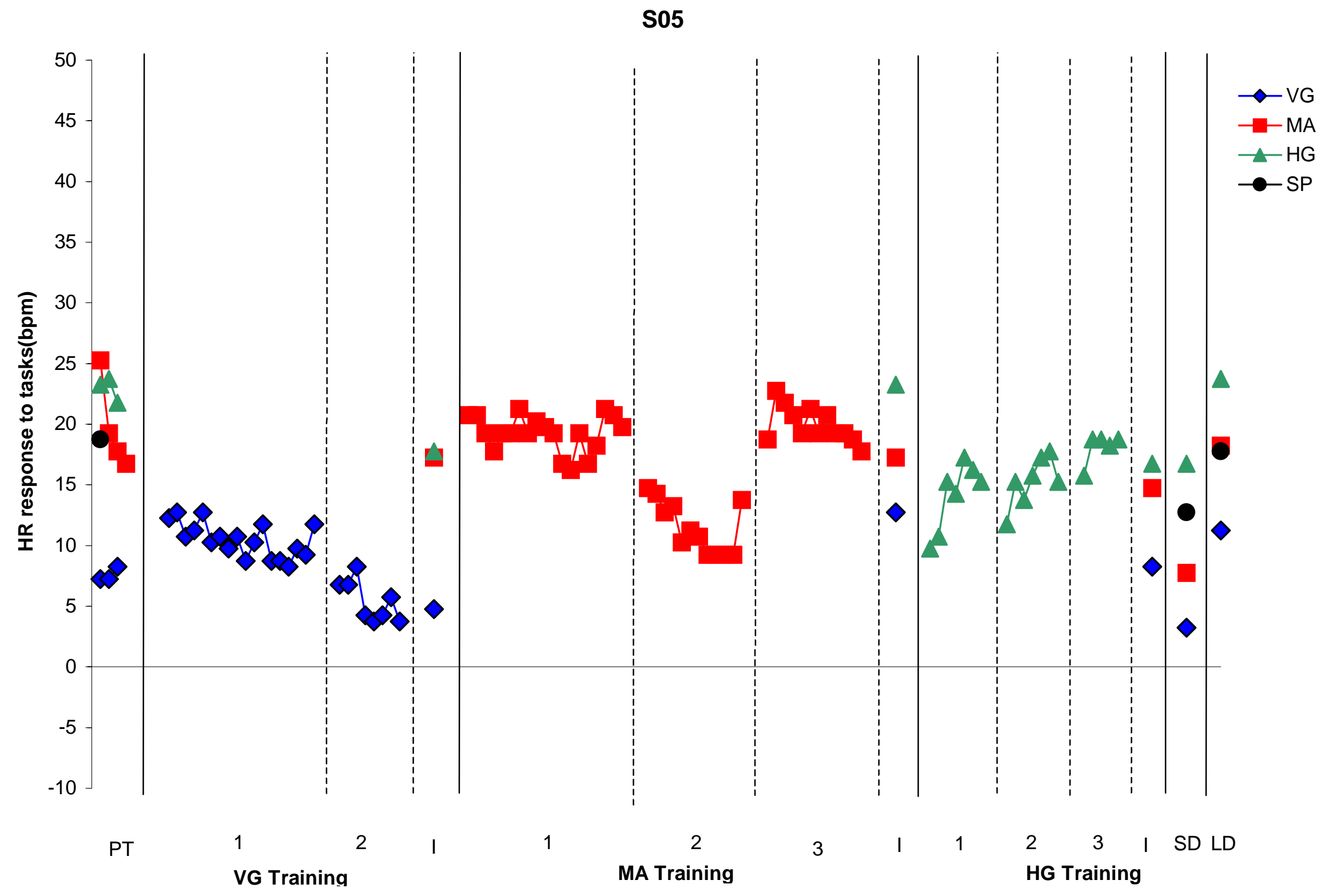


Figure 7.

\section{S10}

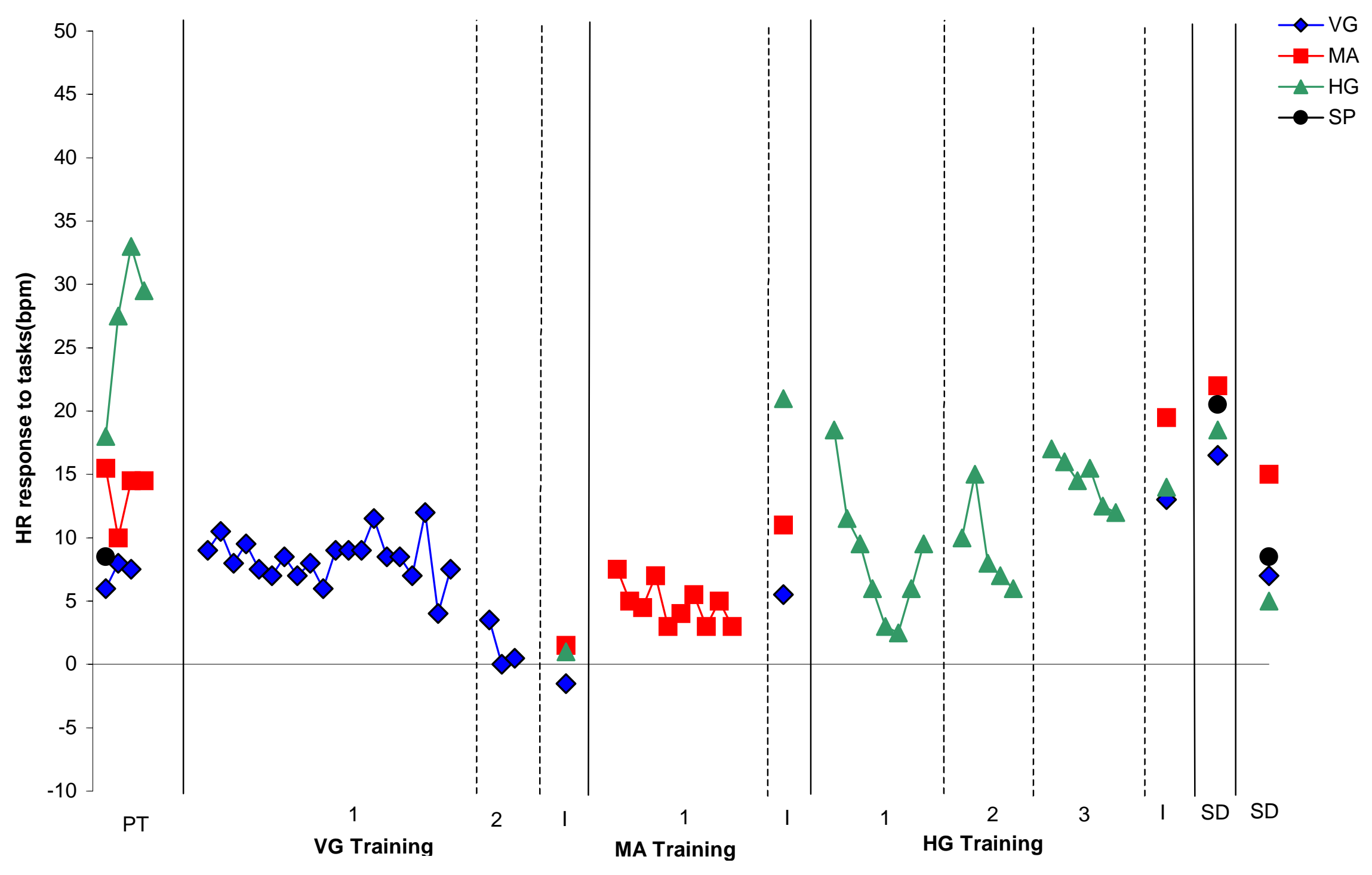


Figure 8.

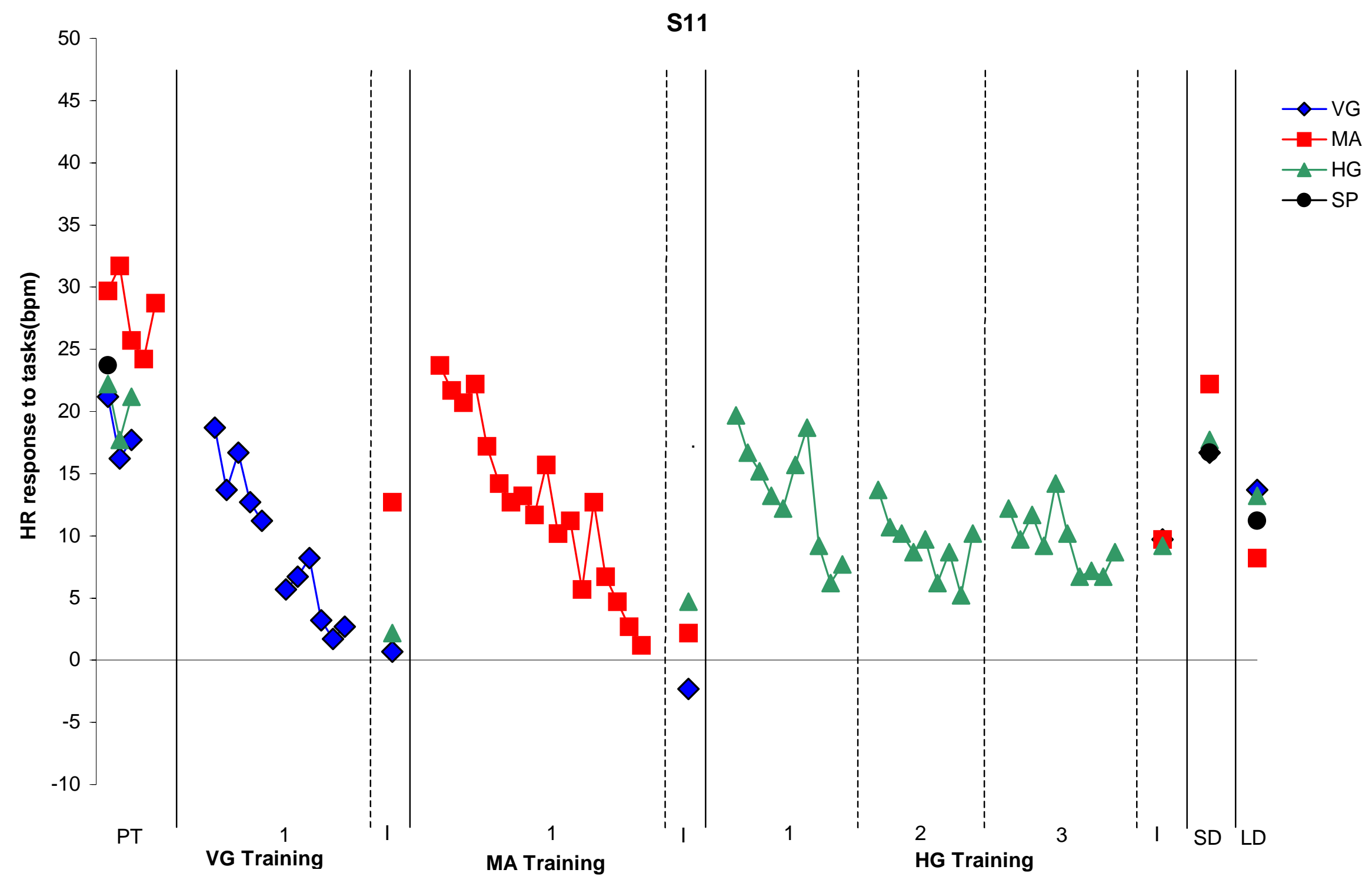




\section{Figure 9.}

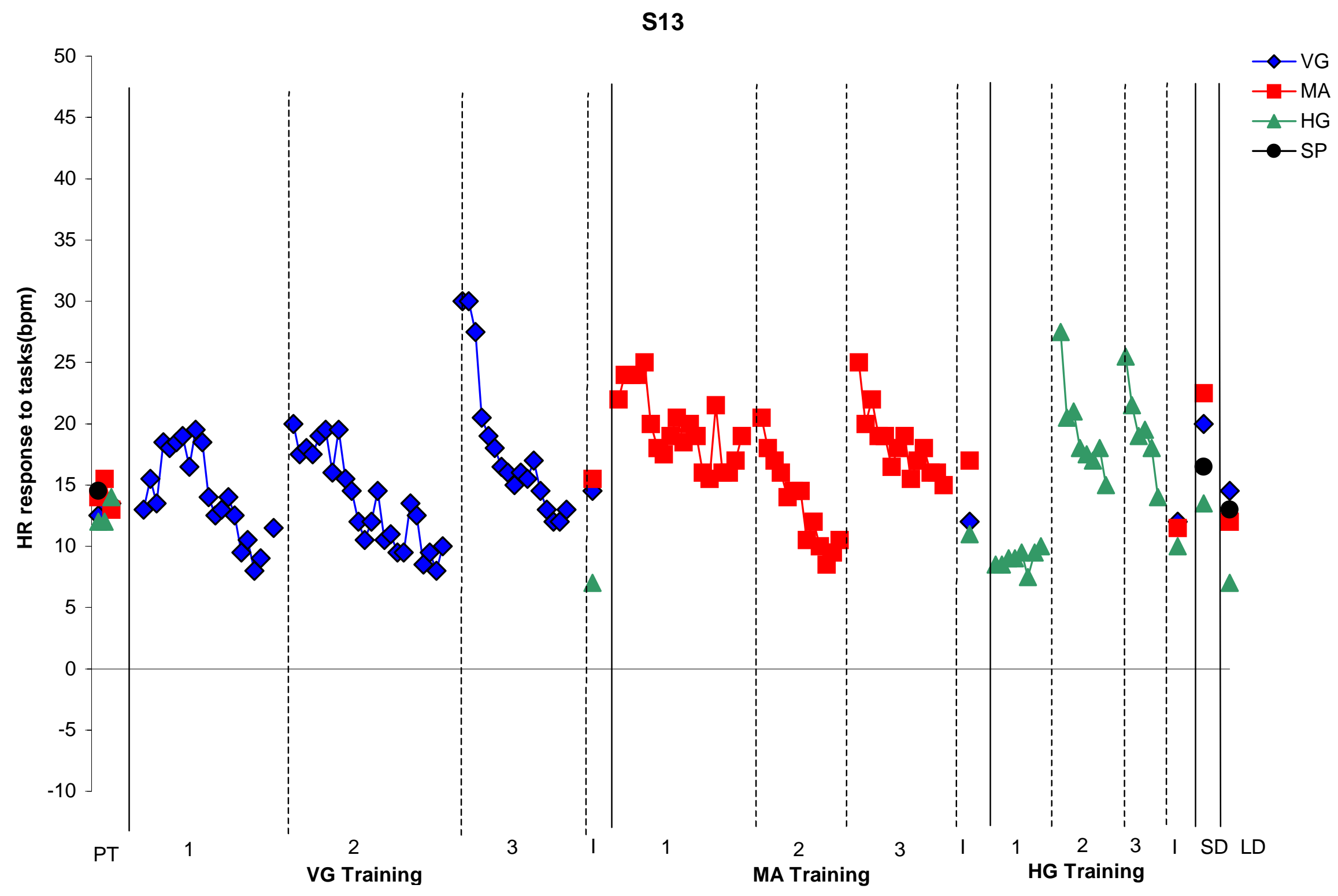


Figure 10.

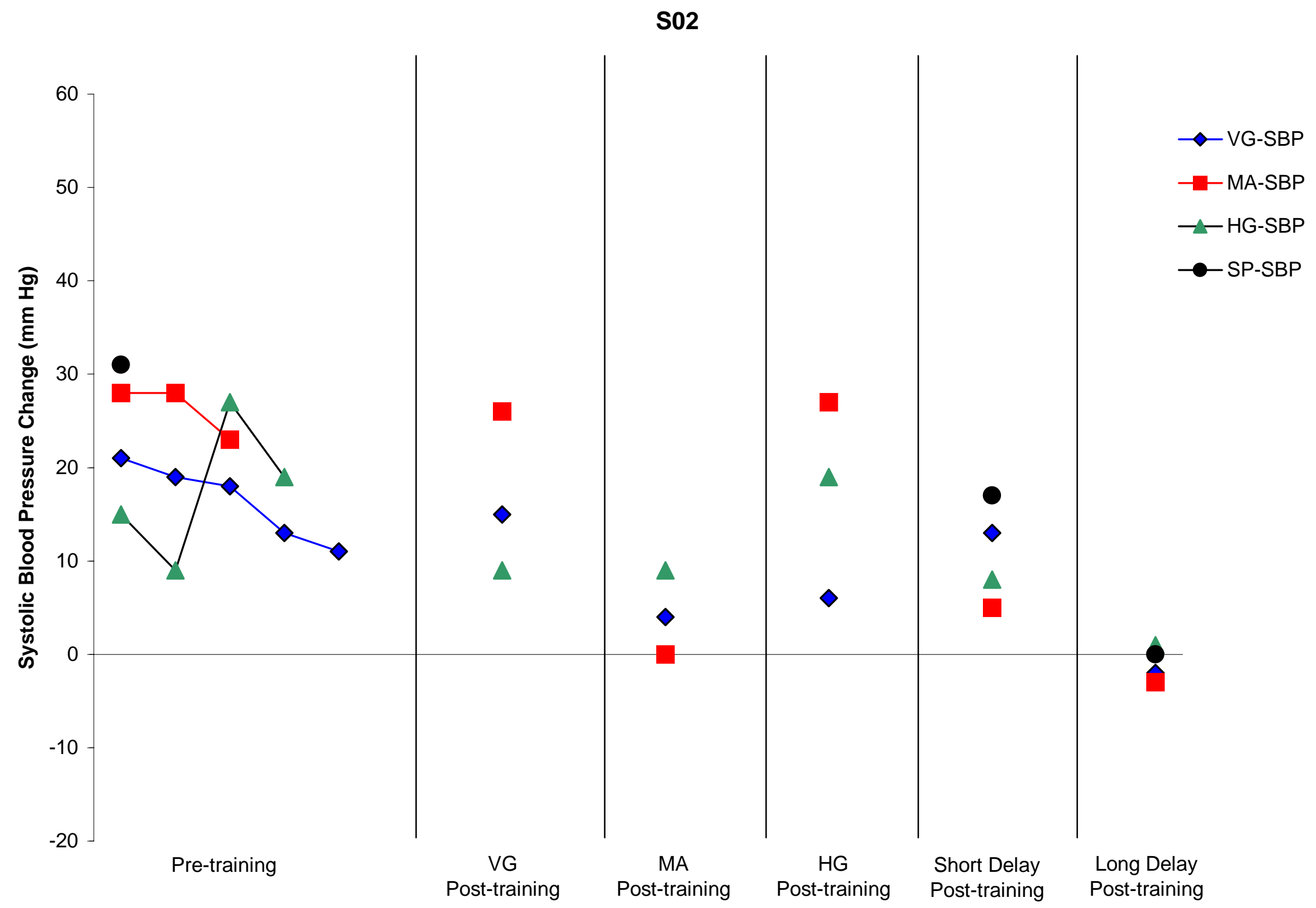


Figure 11.

\section{S04}

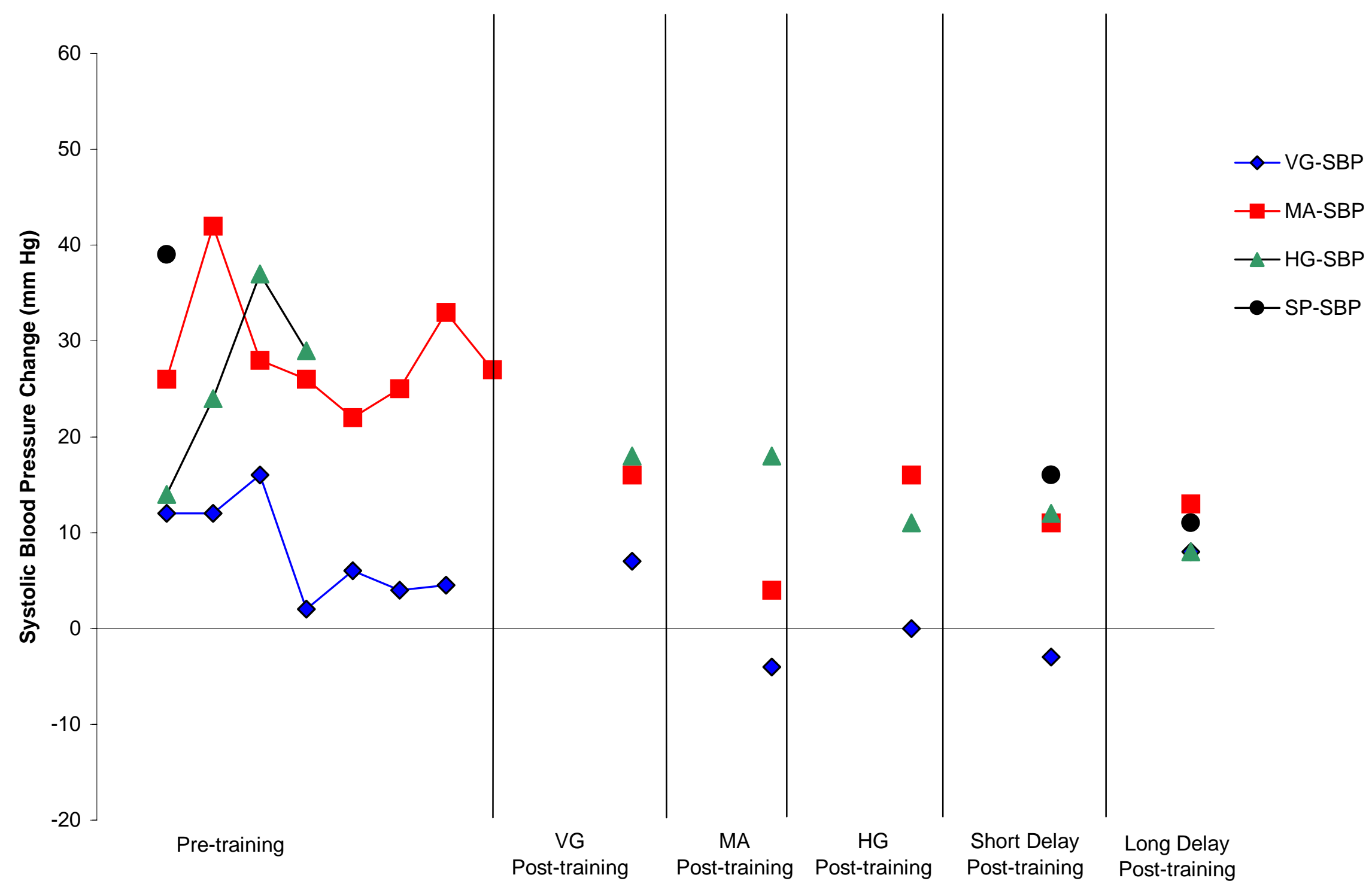


Figure 12.

\section{S05}

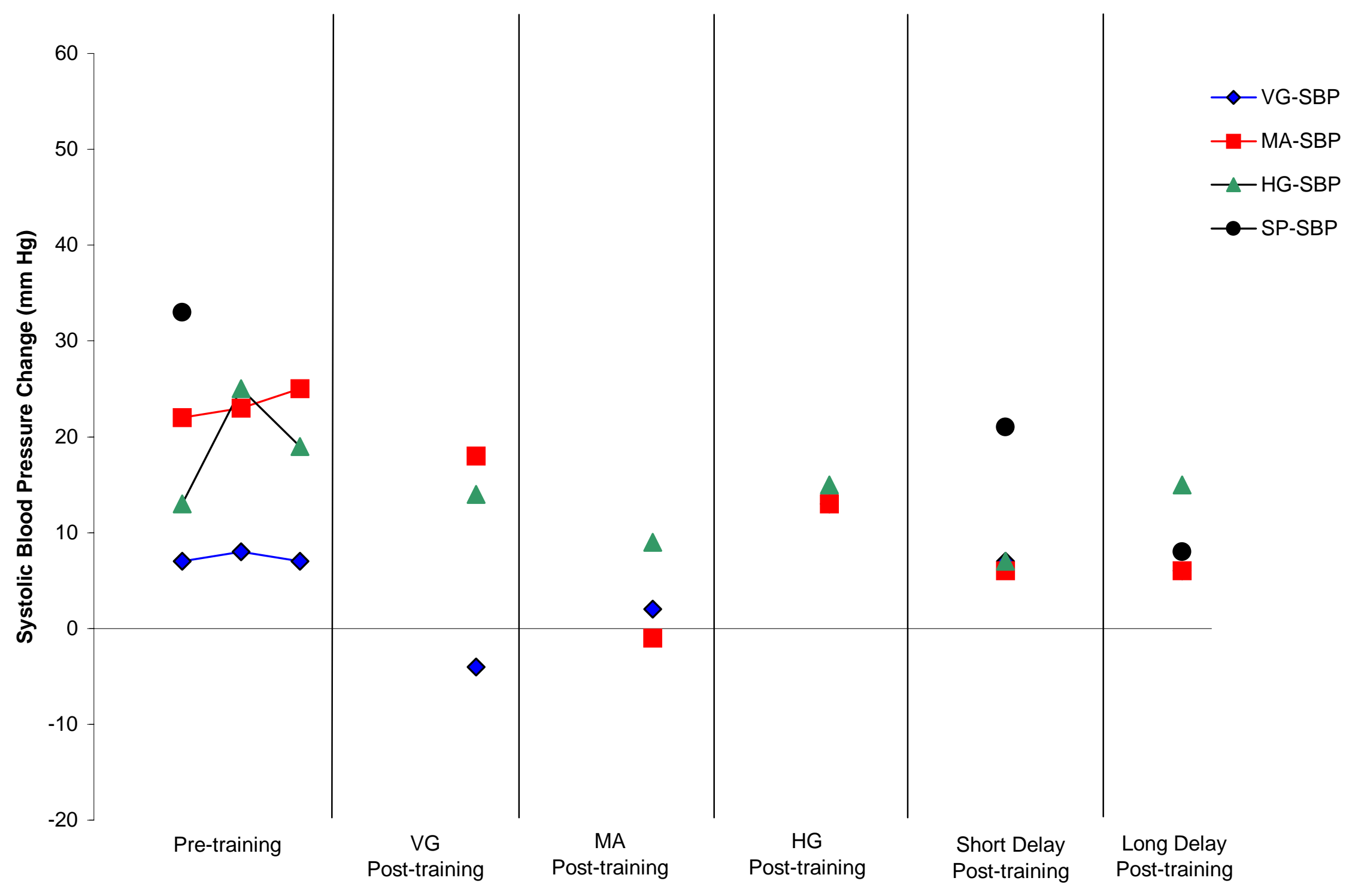


Figure 13.

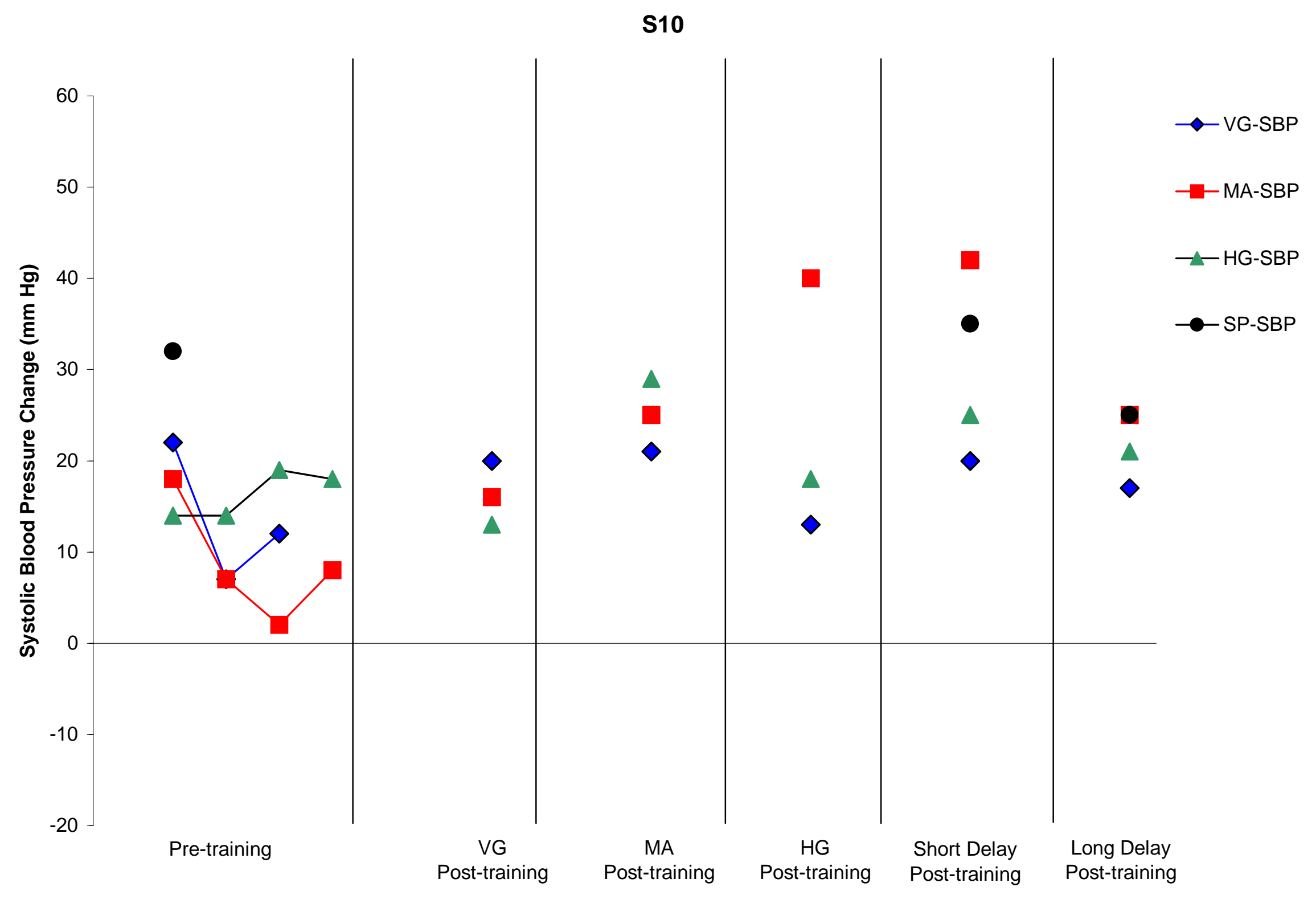


Figure 14.

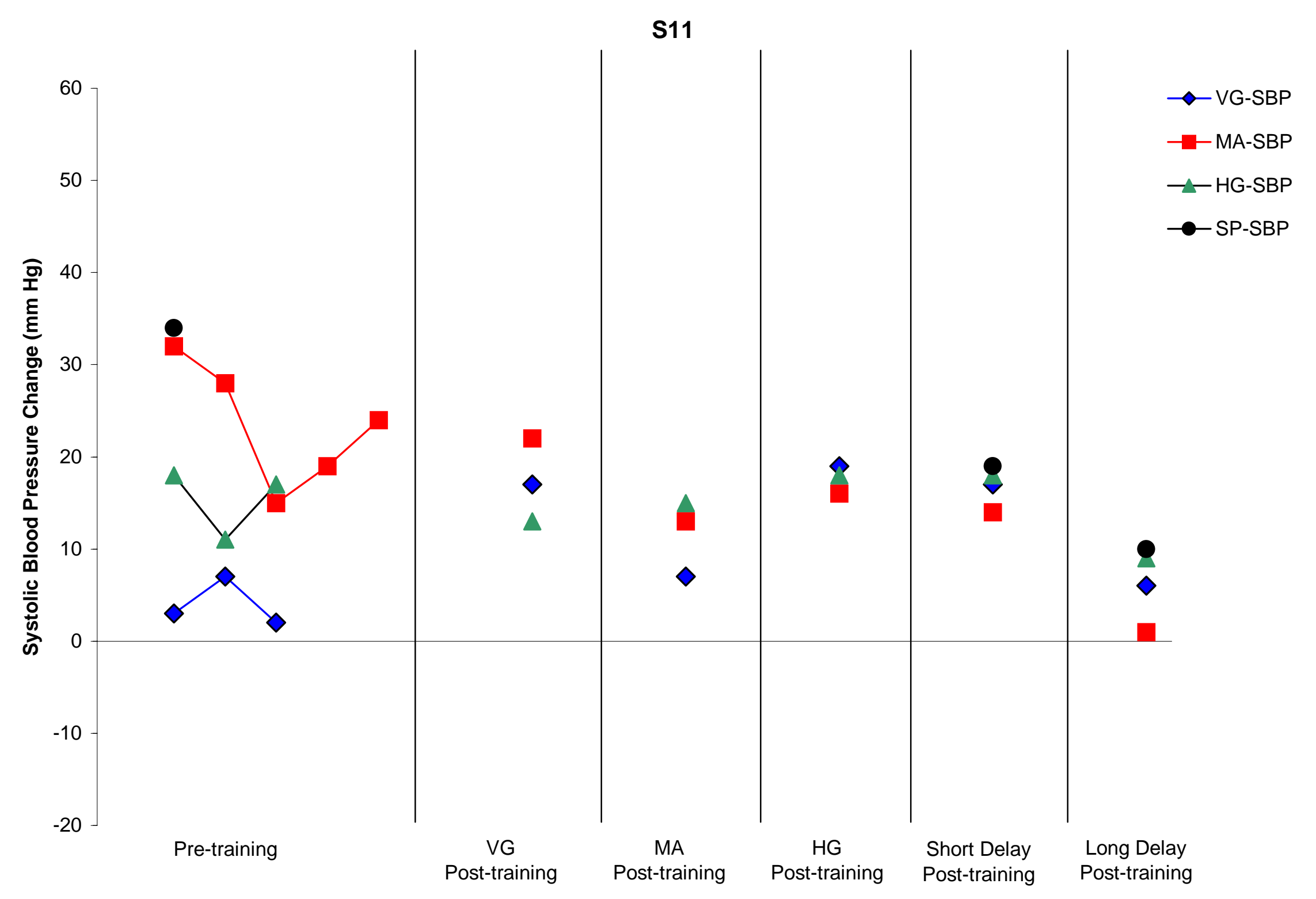


Figure 15.

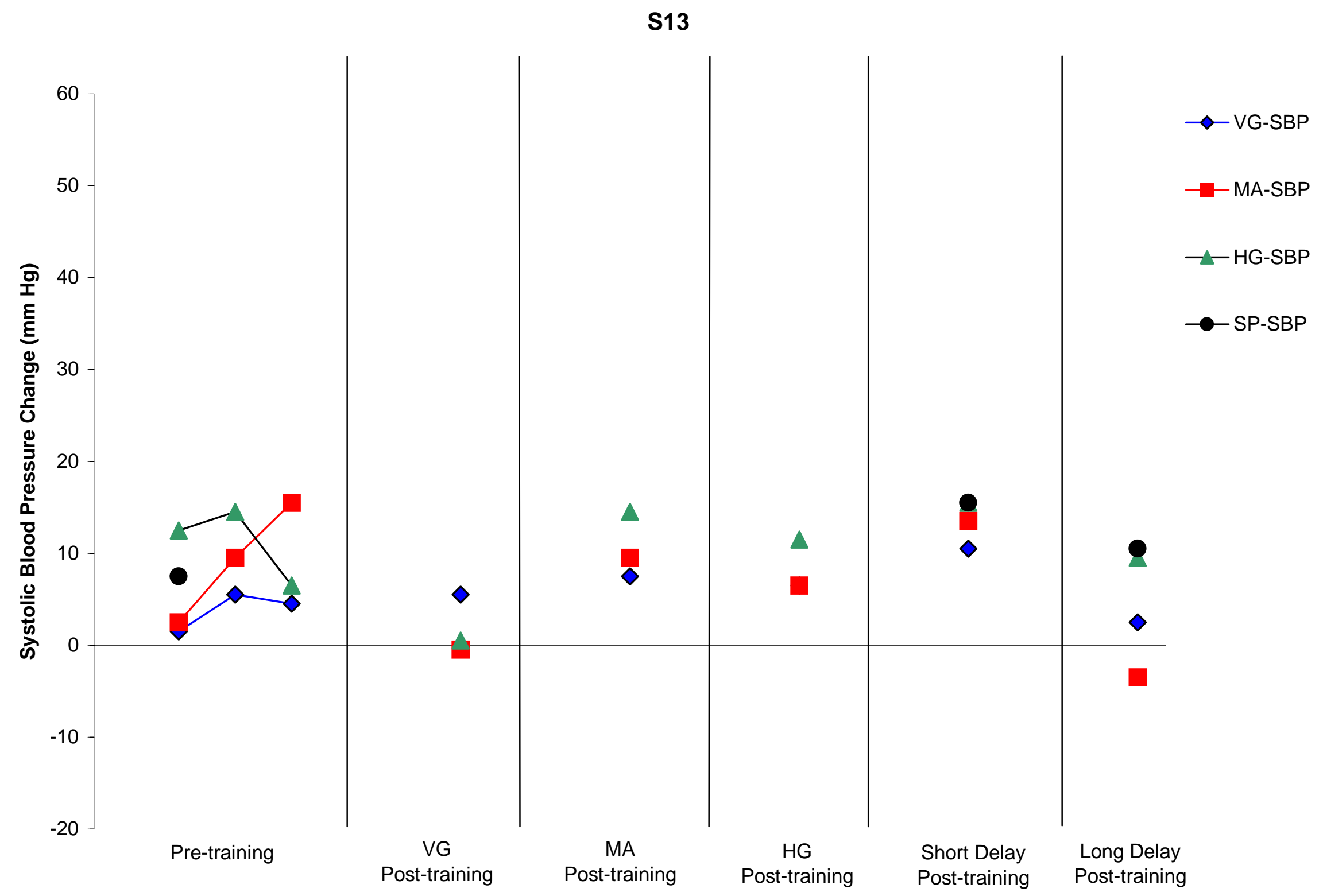


Figure 16.

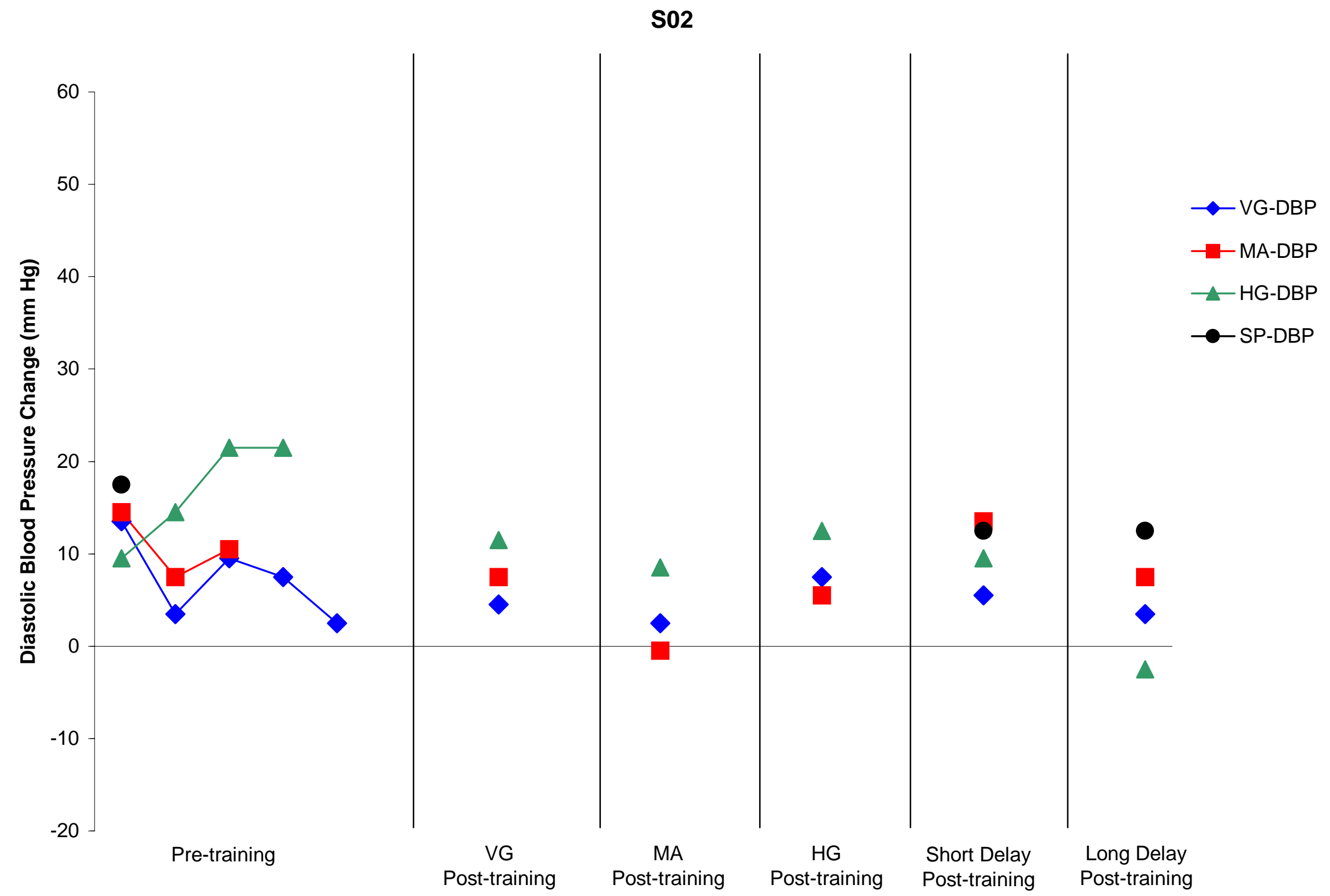


Figure 17.

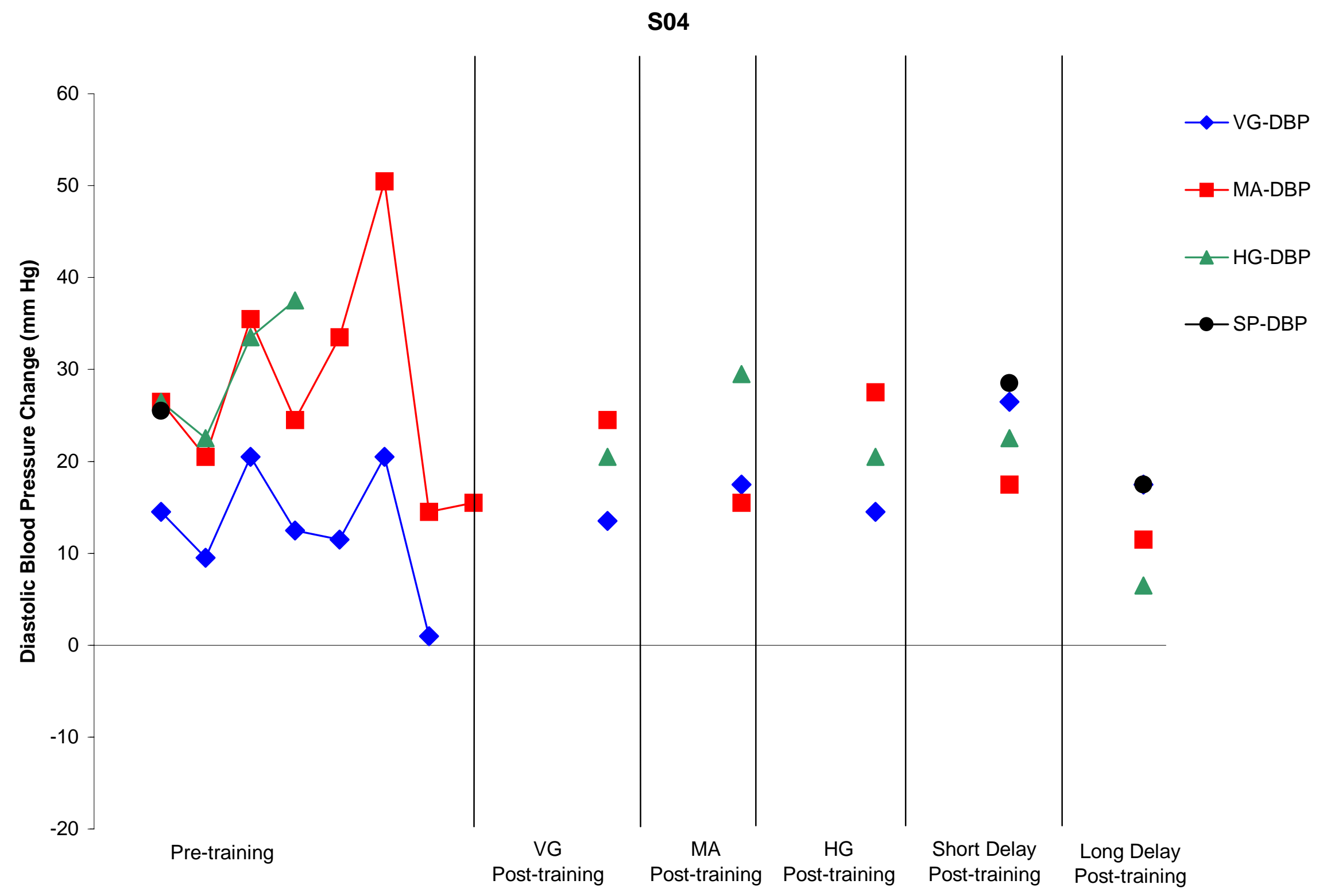


Figure 18.

\section{S05}

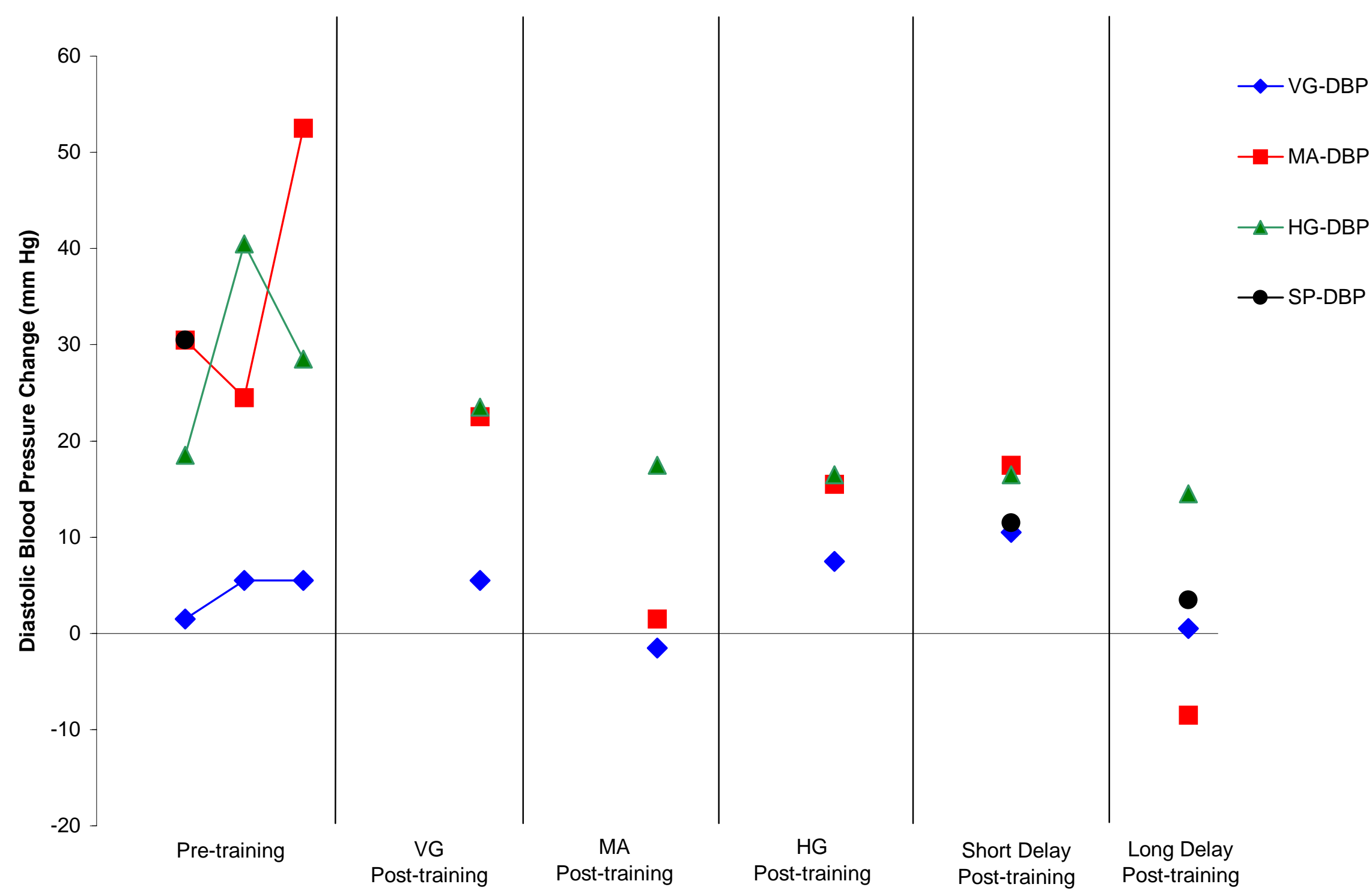


Figure 19.

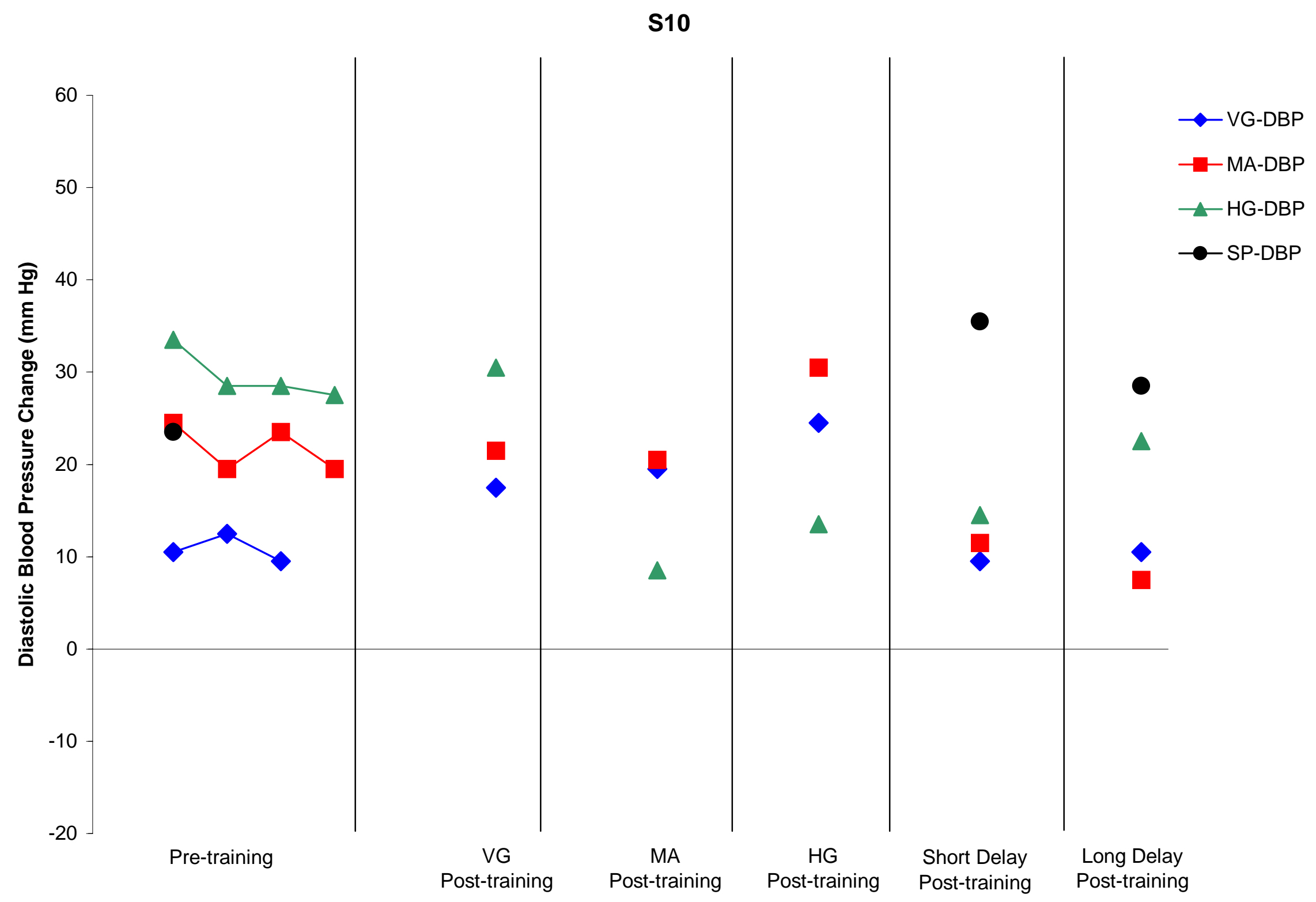


Figure 20.

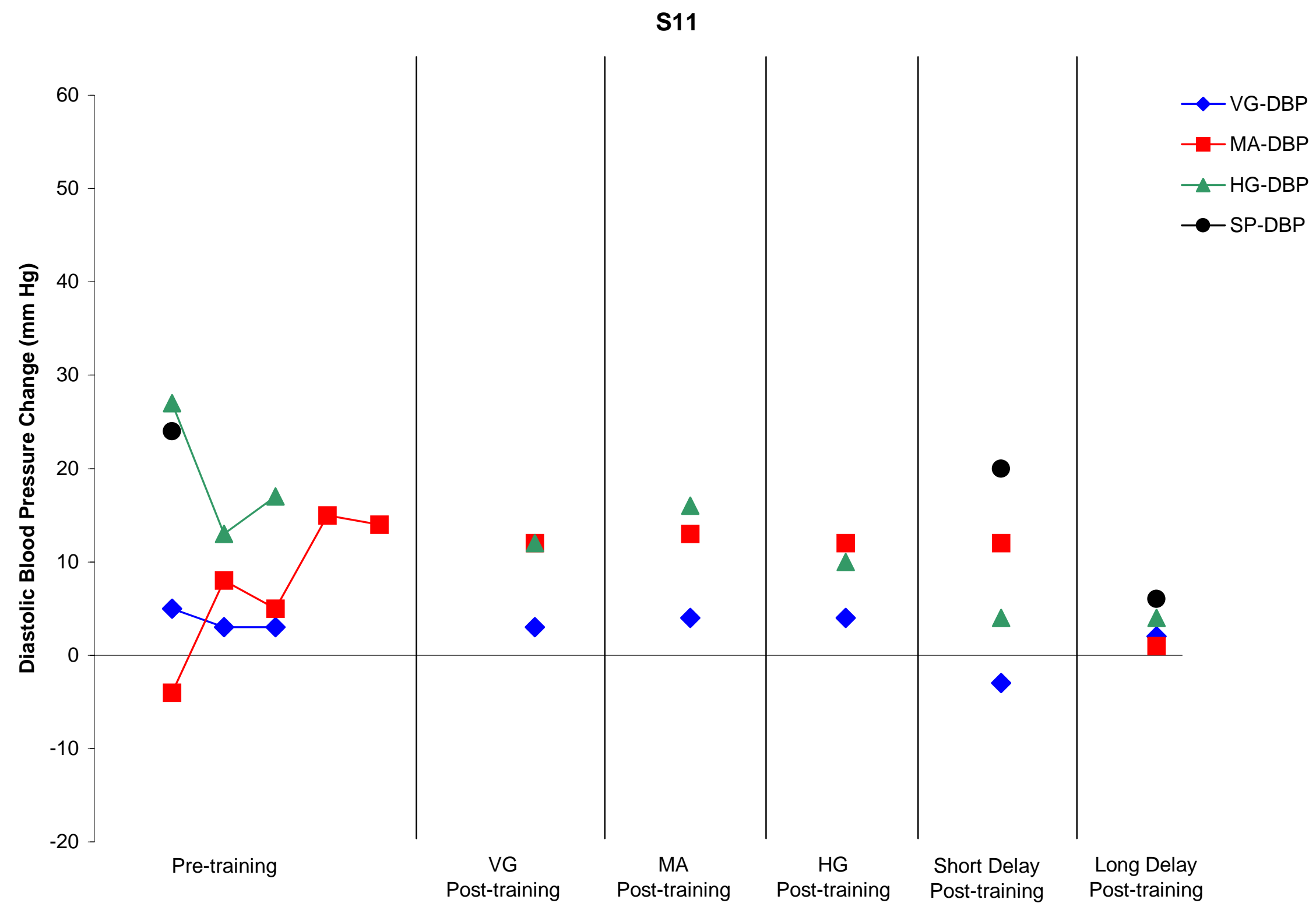


Figure 21.

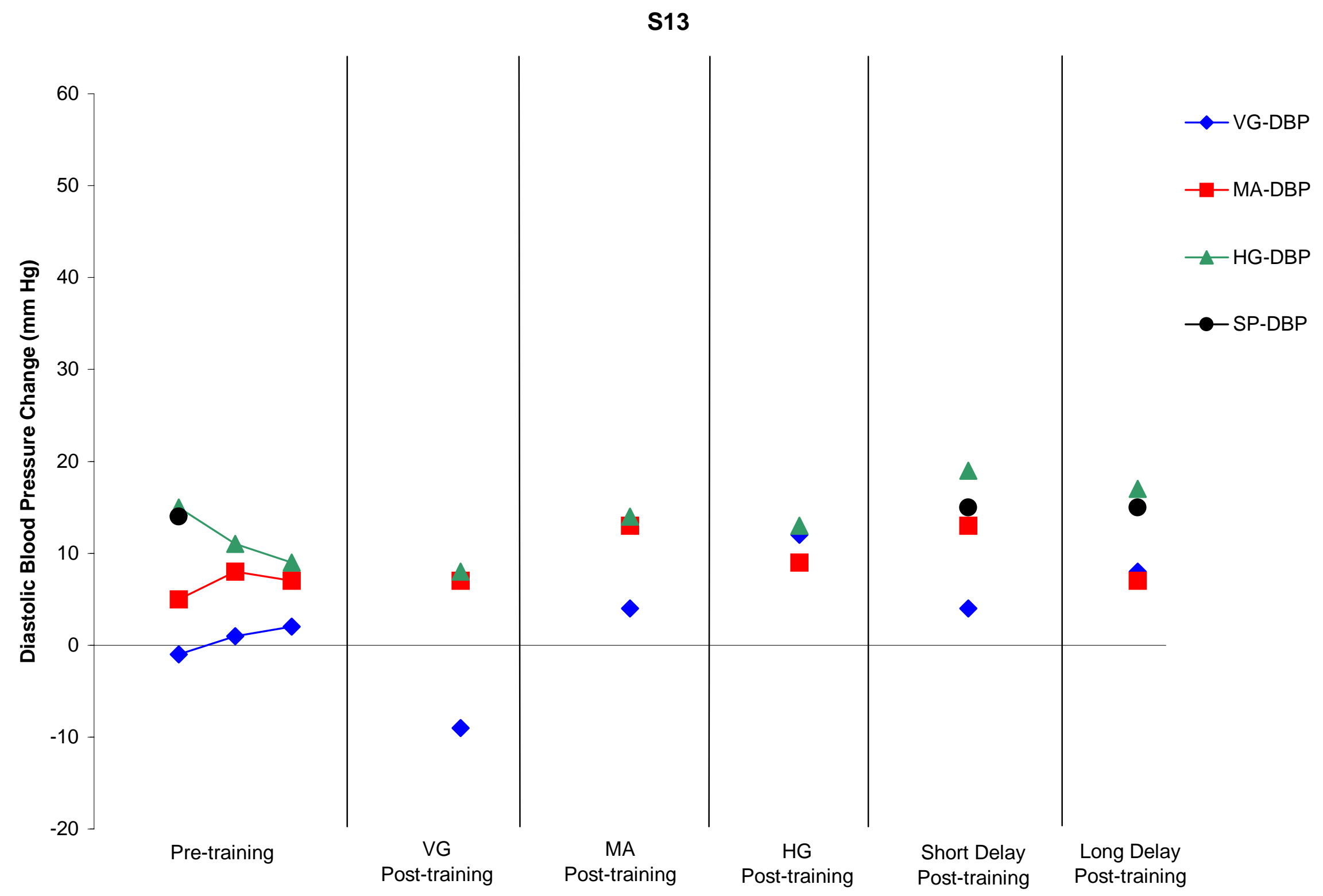


Figure 22.

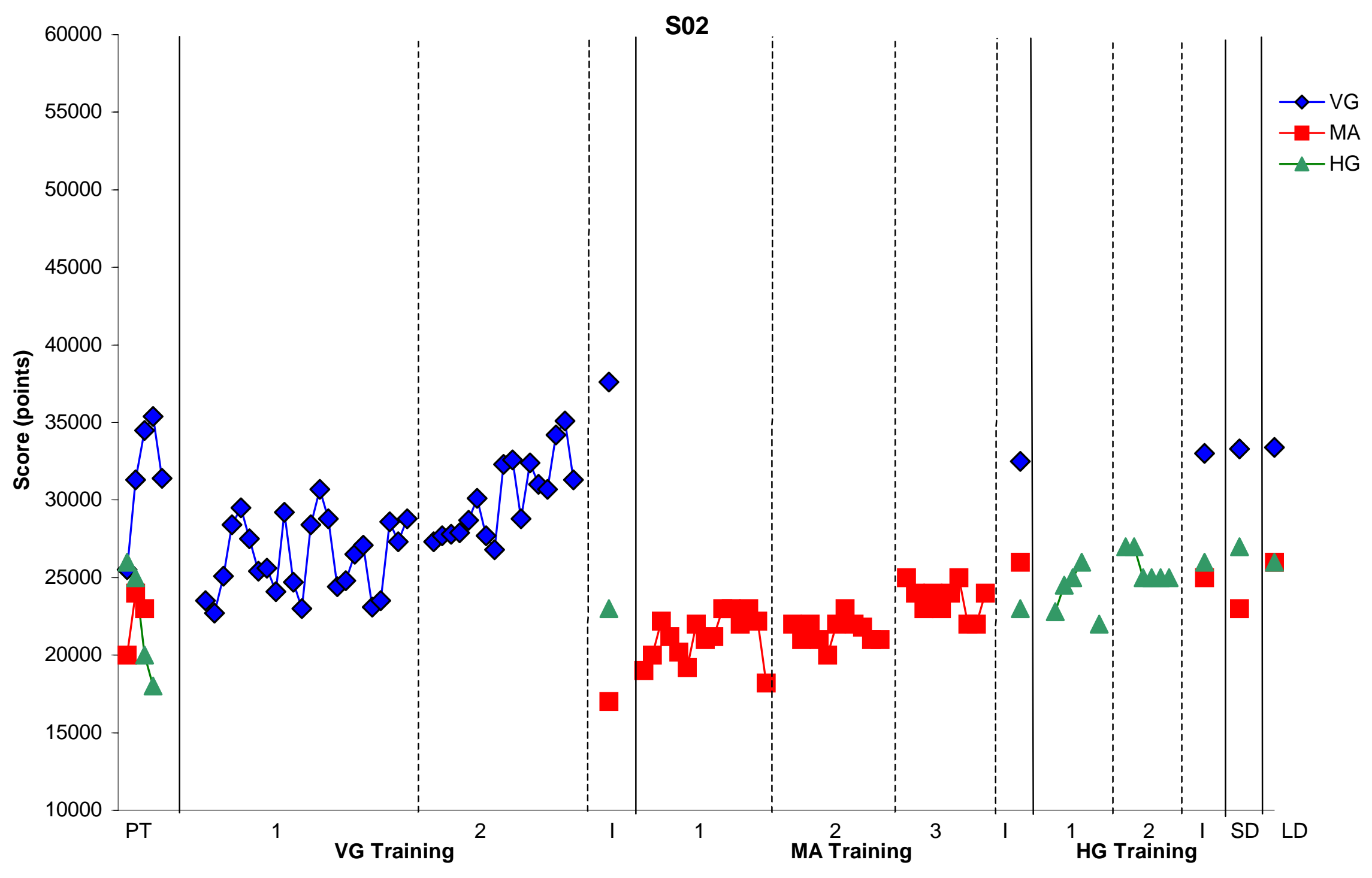


Figure 23.

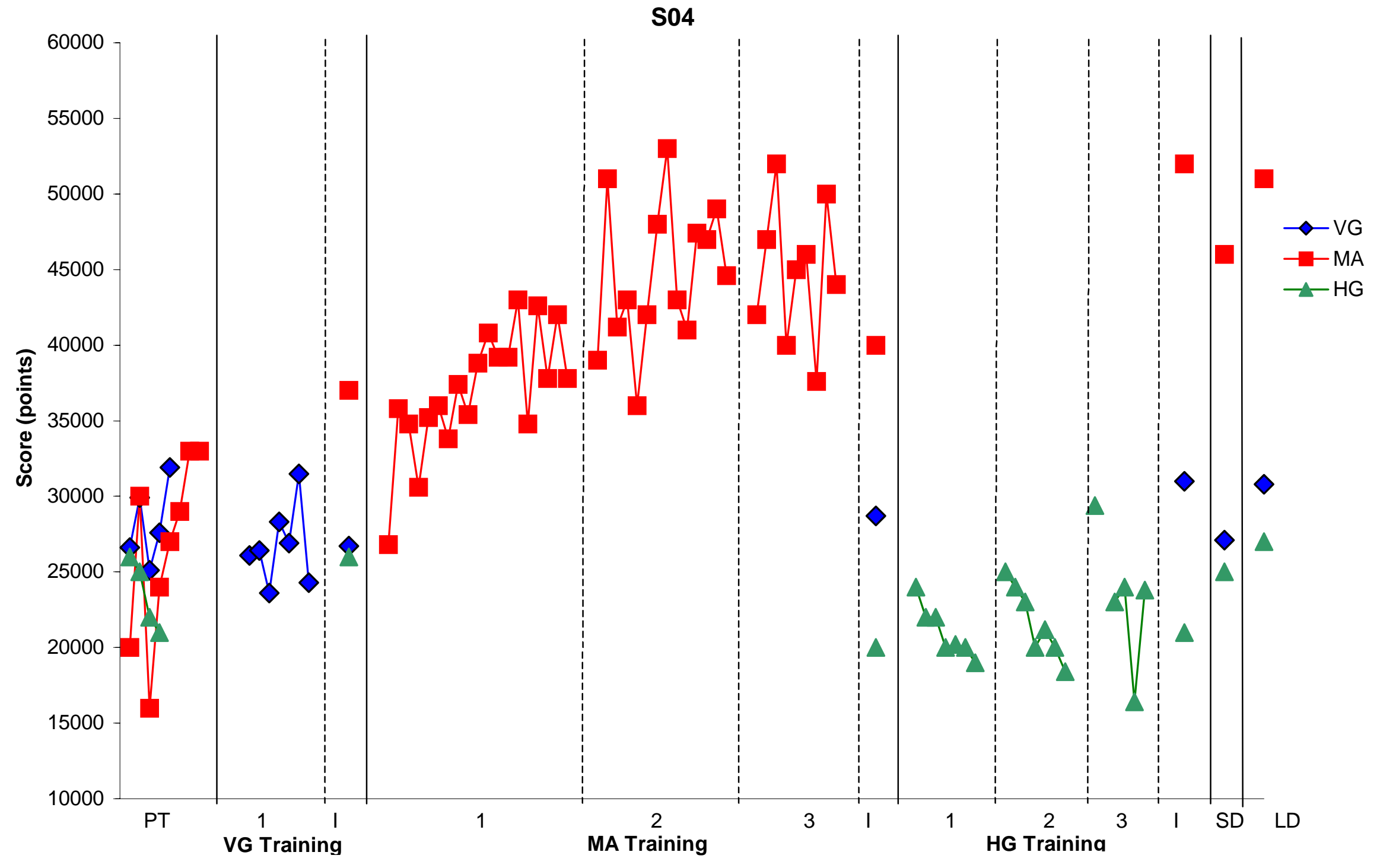


Figure 24.

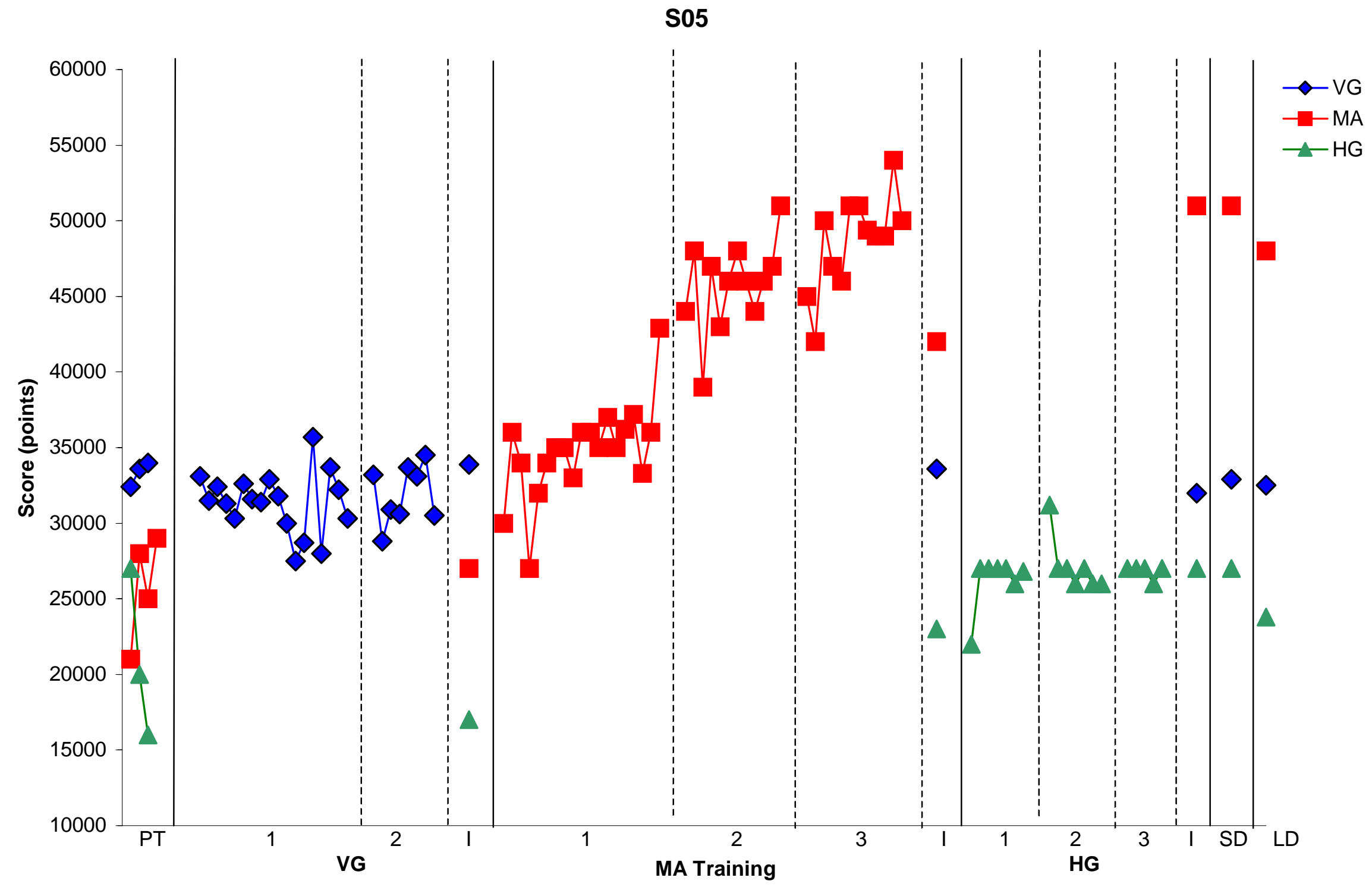


Figure 25.

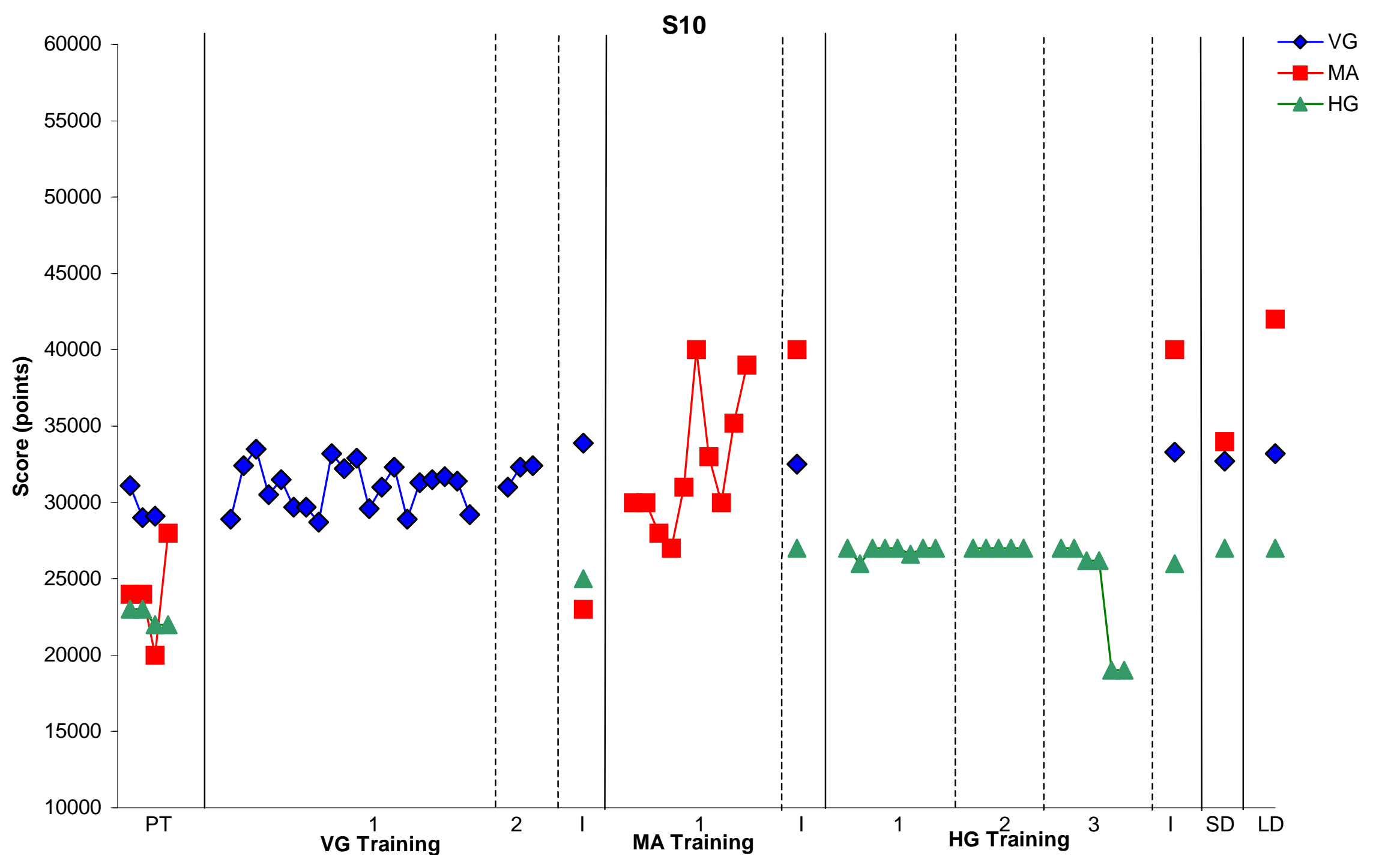


Figure 26.

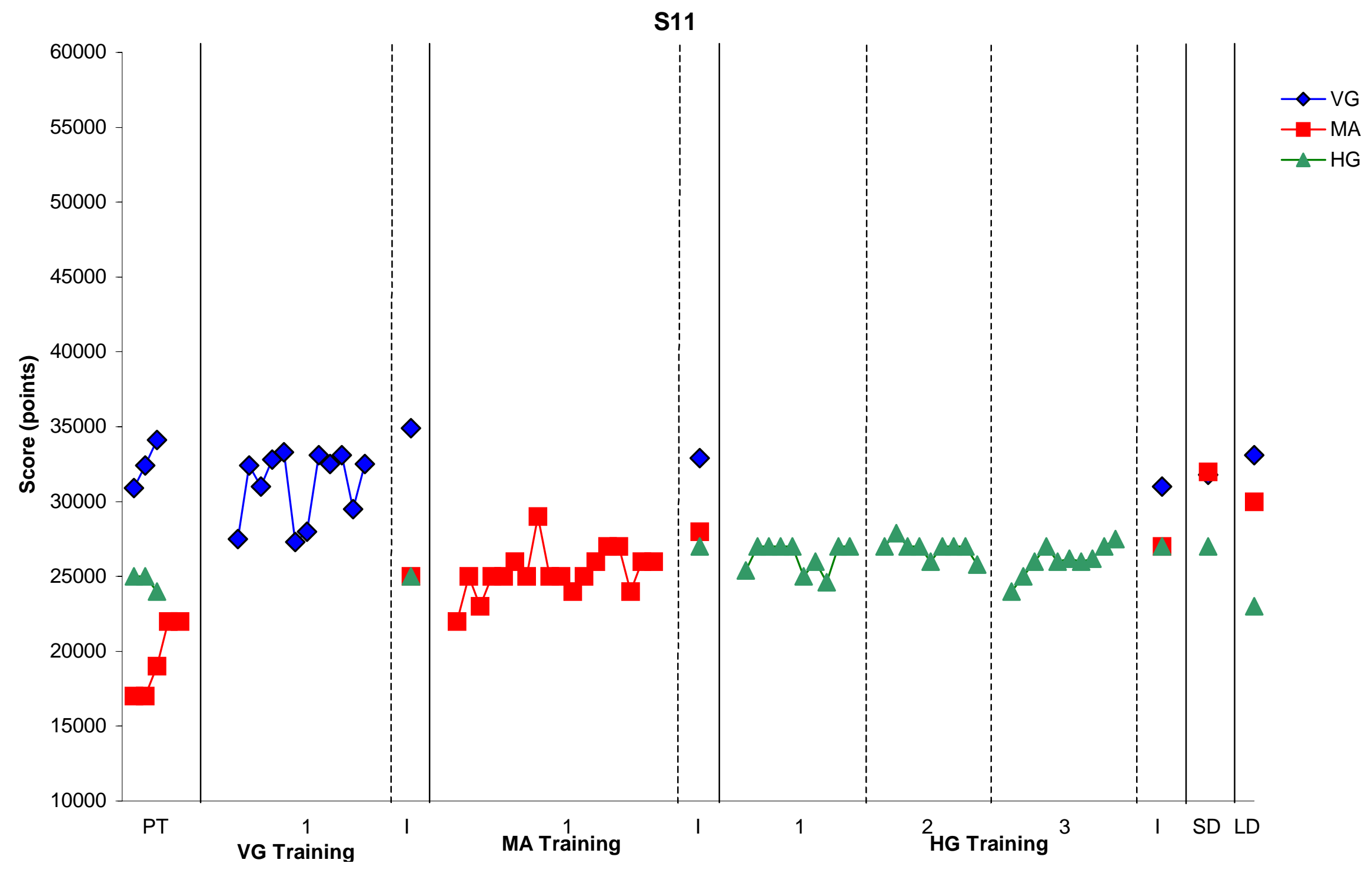


Figure 27.

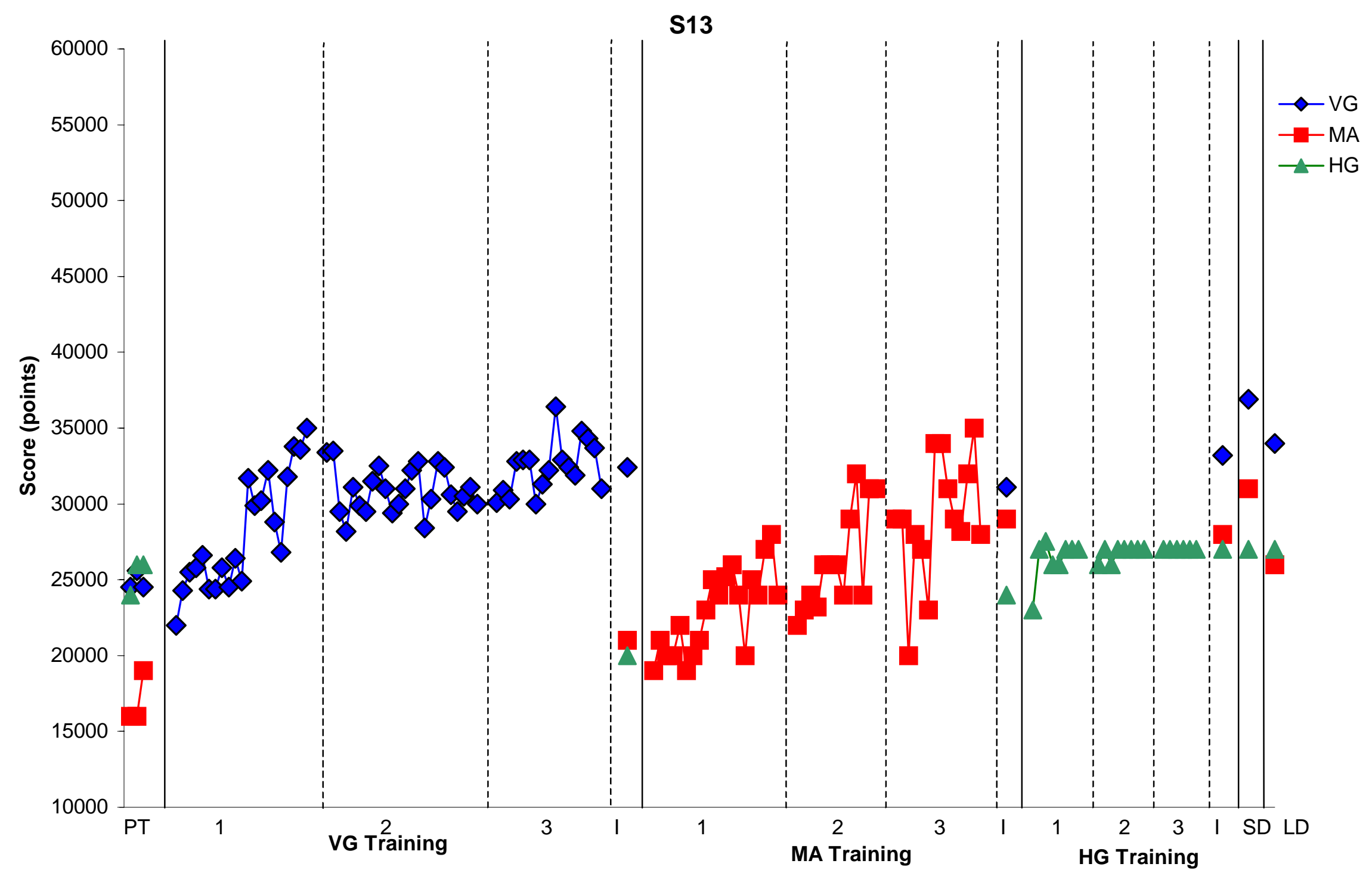


Figure 28.

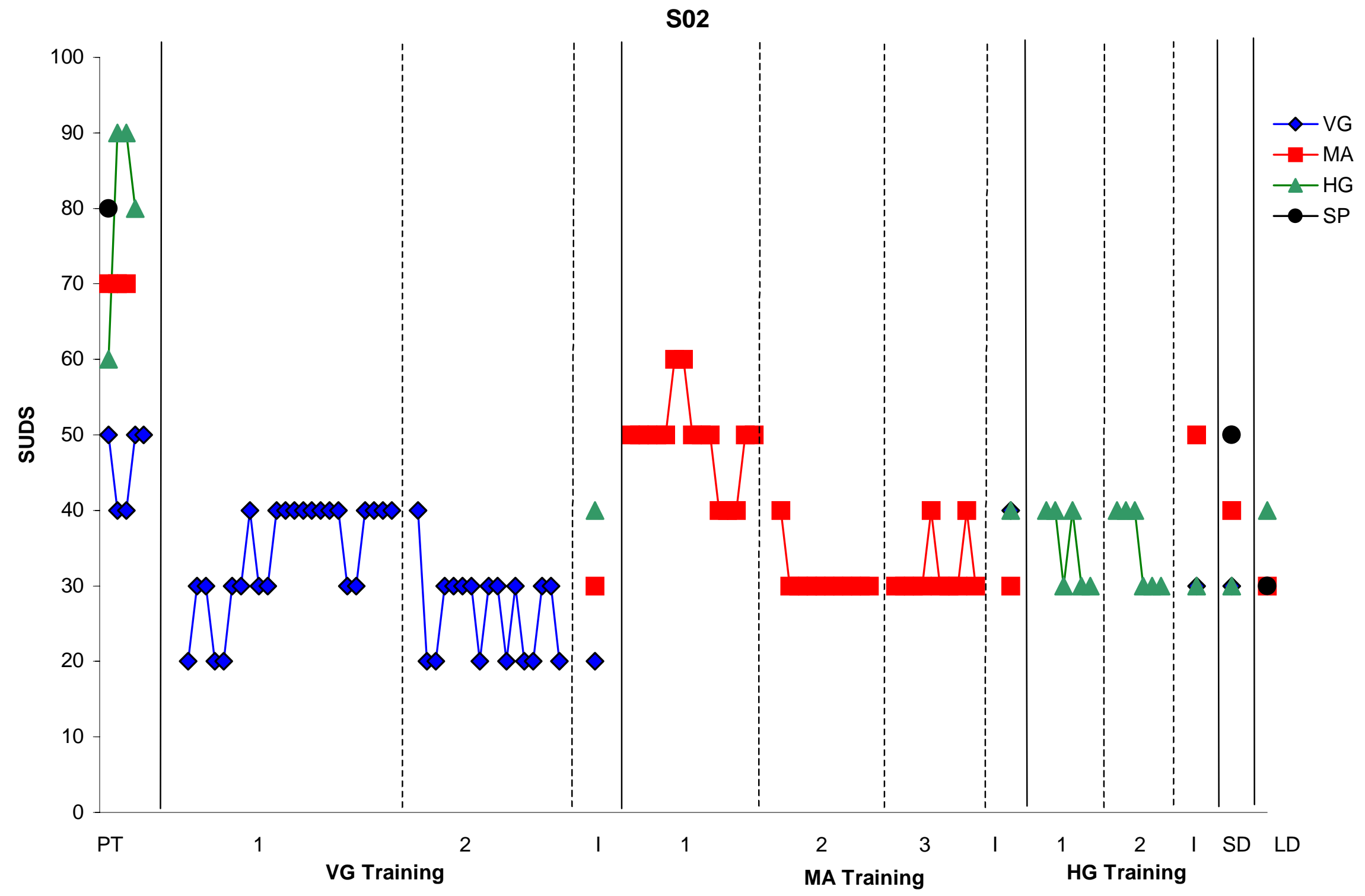


Figure 29.

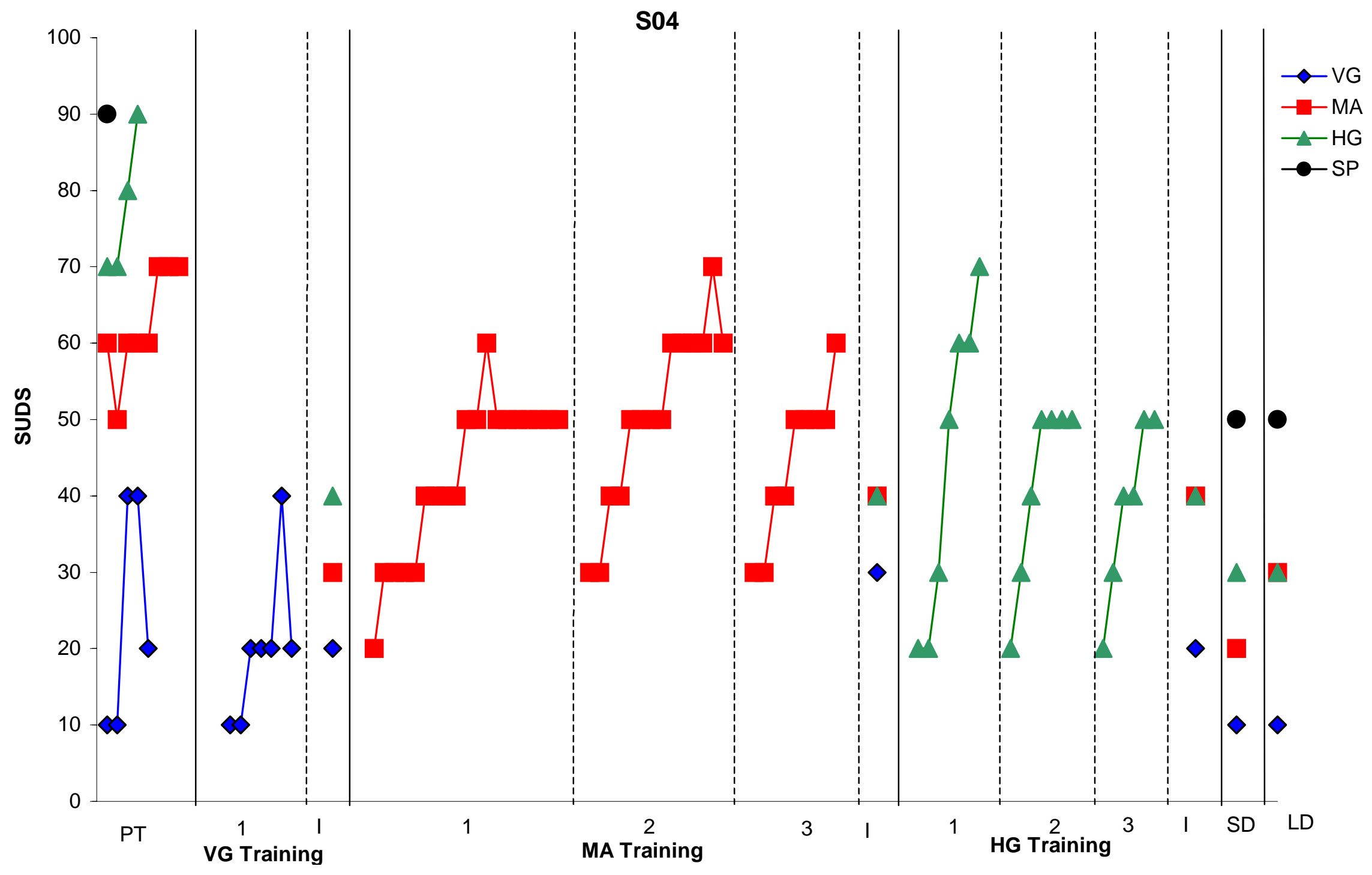


Figure 30.

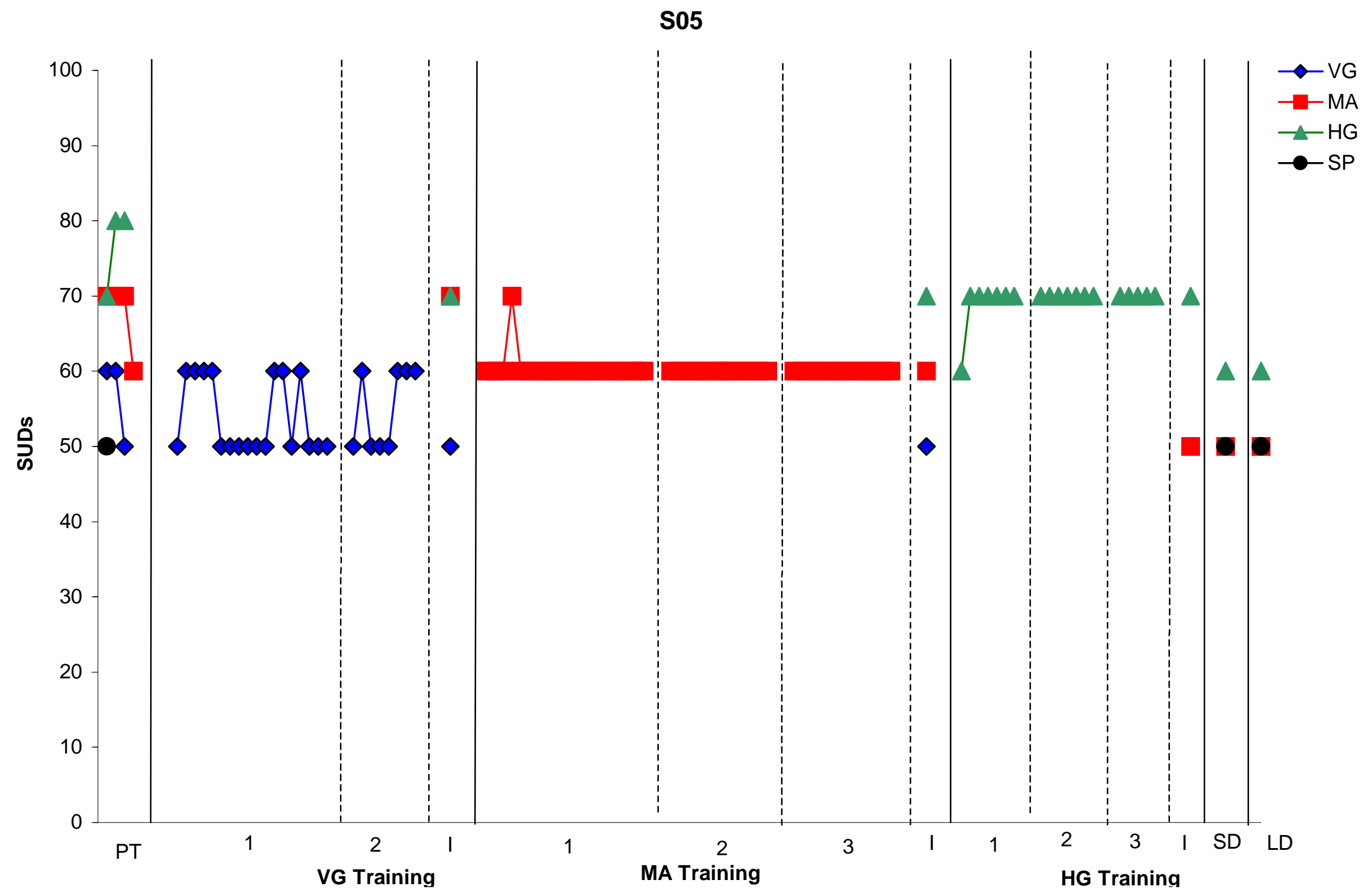




\section{Figure 31.}

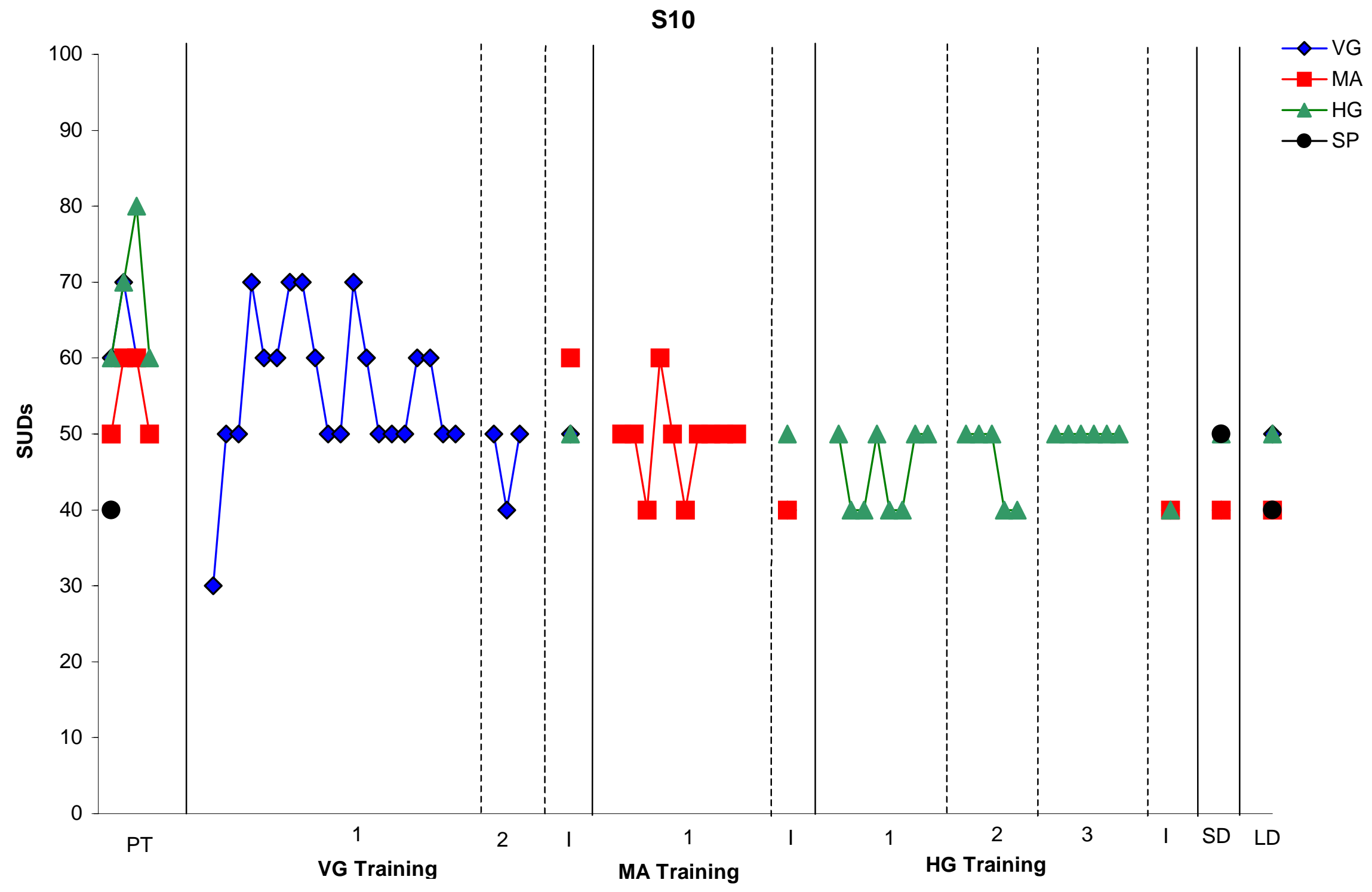


Figure 32.

\section{S11}

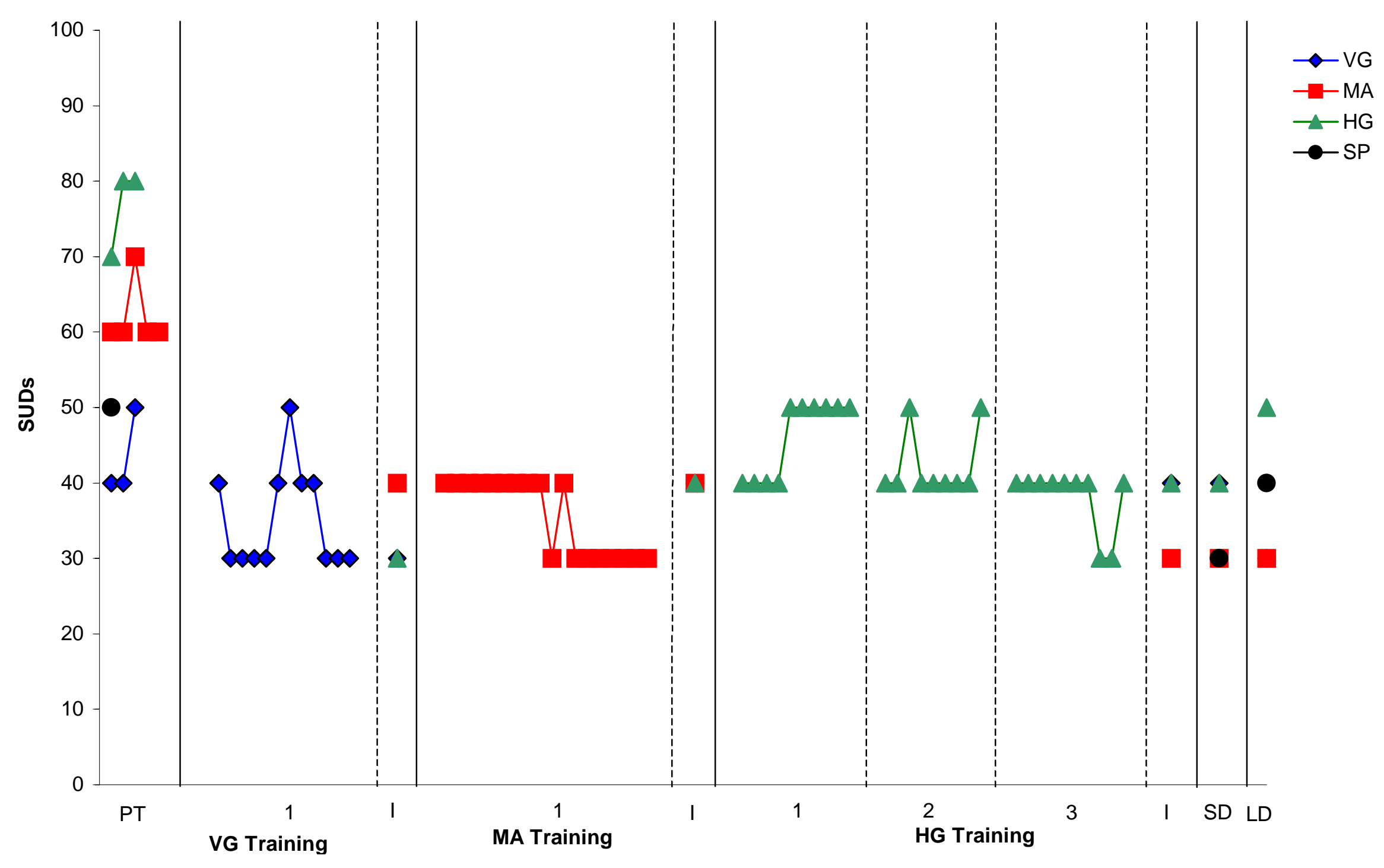


Figure 33.

S13

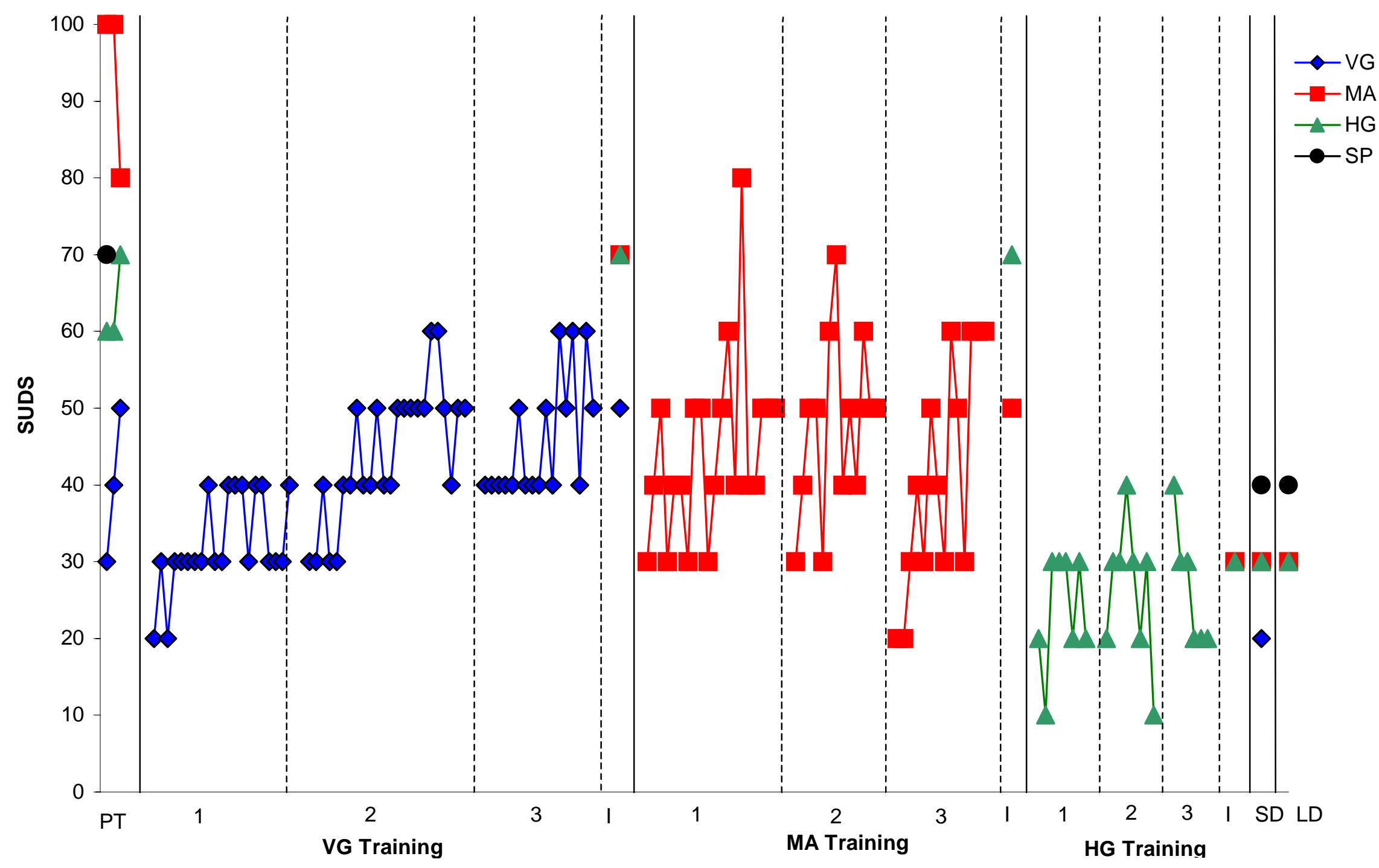




\title{
JEFFREY LOUIS GOODIE
}

\author{
CURRICULUM VITA
}

August 2001

\section{EDUCATION}

Clinical Internship

8/00-Present

Clinical Psychology Intern

Wilford Hall Medical Center, Lackland AFB, TX

Graduate

5/99-Present

Doctoral Candidate

Adult Clinical Psychology Ph.D. Program, West Virginia University

$8 / 96-12 / 98$

Master of Arts degree in Psychology

Adult Clinical Psychology Ph.D. Program, West Virginia University

Undergraduate

8/89-5/93

Bachelor of Arts degree in Psychology

Psychology Major, Dickinson College

Dean's List 1992-1993 (Top 5\% of students for two semesters)

\section{CLINICAL EXPERIENCE}

8/00-Present Psychology Resident

Wilford Hall Medical Center

Lackland AFB, TX

7/98-6/99

Pain Management Practicum Student

Center for Pain Management, Department of Anesthesiology

Morgantown, WV

5/99-6/99

Graduate Student Psychogeriatric Consultant

Department of Psychology

Hopemont Hospital, Terra Alta, WV

8/98-7/99

Graduate Student Therapist

Quin Curtis Center, Department of Psychology

West Virginia University, Morgantown, WV

8/97-6/98

Inpatient Psychogeriatric Practicum Student

Department of Psychology

Hopemont Hospital, Terra Alta, WV 
Graduate Student Therapist

Quin Curtis Center, Department of Psychology

West Virginia University, Morgantown, WV

$5 / 97-8 / 97$

Inpatient/Outpatient Practicum Student

Louis A. Johnson Veterans Memorial Hospital

Clarksburg, WV

$10 / 95-6 / 96$

Mental Health/Mental Retardation Technician

Personal-Touch

Pittsburgh, PA

$6 / 95-7 / 96$

Volunteer Psychiatric and Research Assistant

Western Psychiatric Institute and Clinic

University of Pittsburgh Medical School, Pittsburgh, PA

\section{RESEARCH EXPERIENCE}

7/99- $7 / 00$

\section{Graduate Research Assistant}

Pathophysiological and Behavioral correlates of Isolated Clinic

Normotension

Department of Psychology

West Virginia University, Morgantown, WV

$5 / 99-5 / 00$

Co-Investigator

Anxiety and Cardiovascular Reactivity to Laboratory Stressors

Department of Psychology

West Virginia University, Morgantown, WV

6/98-3/00

Research Assistant

Neuropsychological Effects of Adult-Residual ADD

Department of Behavioral Medicine and Psychiatry

Chestnut Ridge Hospital, Morgantown, WV

$8 / 93-7 / 96$

\section{Research Specialist II}

Ambulatory Blood Pressure Monitoring and Daily Stress

Department of Psychology

University of Pittsburgh, Pittsburgh, PA

\section{TEACHING EXPERIENCE}

$1 / 00-5 / 00$

Instructor, Health Psychology

West Virginia University, Morgantown, WV 
$1 / 99-5 / 99 \&$

$1 / 00-5 / 00$

$8 / 98-12 / 98$

$8 / 98-12 / 98$

$7 / 98$

$1 / 98$

$8 / 97-12 / 97$

$11 / 97 \& 6 / 98$

$8 / 96-5 / 97$
Graduate Teaching Assistant, Behavioral Assessment II West Virginia University, Morgantown, WV

Graduate Teaching Assistant, Introduction to Clinical Psychology West Virginia University, Morgantown, WV

Graduate Teaching Assistant, Child Behavior Modification West Virginia University, Morgantown, WV

Workshop Co-Presenter, Psychological Factors Associated with Medical Disorders, Hopemont Hospital, Nursing staff

Workshop Co-Presenter, Aggression in Alzheimer's Patients

Alzheimer's Caregivers Support Group

Graduate Teaching Assistant, Introduction to Clinical Psychology West Virginia University, Morgantown, WV

Workshop Co-Presenter, Behavior Modification with Older Adults Hopemont Hospital

Course Instructor, Experimental Analysis of Behavior

West Virginia University, Morgantown, WV

\section{PUBLICATIONS}

Frazer, N., Larkin, K. T., \& Goodie, J. L., (in press). The relation between cardiovascular reactivity to stress and parental history of hypertension: Examination of covert and overt responses to stressors. Health Psychology.

Goodie, J. L., \& Larkin, K. T. (in press). Changes in hemodynamic response to mental stress with heart rate feedback training. Applied Psychophysiology and Biofeedback.

Zvolensky, M. J., Goodie, J. L., Ruggiero, K. J., Black, A. L., Larkin, K. T., \& Taylor, B. K. (in press). Perceived stress and anxiety sensitivity in the prediction of anxiety-related responding: A multichallenge evaluation. Anxiety, Stress, and Coping: An International Journal.

Davig, J., Larkin, K. T., \& Goodie, J. L. (2001). Does cardiovascular reactivity to stress measured in the laboratory generalize to thesis and dissertation meetings among doctoral students? International Journal of Behavioral Medicine.

Goodie, J. L., Larkin, K. T., \& Schauss, S. (2000). Validation of the Polar heart rate monitor for assessing heart rate during physical and mental stressors. Journal of Psychophysiology, 14, 159-164. 
Zvolensky, M. J., Goodie, J. L., McNeil, D. W., Sperry, J. A., \& Sorrell, J. T. (in press). Anxiety sensitivity in the prediction of pain-related fear and anxiety in a heterogeneous chronic pain population. Behaviour Research and Therapy.

Edelstein, B., Goodie, J. L., \& Martin, R. (2000). Physiological and behavioral concomitants of aging. In R. Corsini, (Ed.), Encyclopedia of Psychology. New York: John Wiley and Sons.

Edelstein, B., Martin, R., \& Goodie, J. L. (2000). Brief therapy with older adults: Assessment and treatment issues. In M. Hersen, \& M. Biaggio, (Eds.), Effective brief treatment for adults: A clinician's guide.

Ruggerio, K. J., Goodie, J. L., \& Morris, T. (1999). Using item analysis to facilitate interpretation of empirical findings. Behavior Therapy and Experimental Psychiatry, 30, 63-69.

Kamarck, T., Shiffman, S., Smithline, L., Goodie, J., Paty, J., Gnys, M., \& Jong, J. (1998). The effects of task strain, social conflict, and emotional activation on ambulatory cardiovascular activity: Daily life consequences of 'recurring stress' in a multiethnic adult sample, Health Psychology, 17, 17-29.

Kamarck, T. Shiffman, S., Smithline, L., Goodie, J., Thompson, H., Ituarte, P., Jong, J., Pro, V., Paty, J., Kassel, J., Gnys, M., \& Perz, W. (1998). The diary of ambulatory behavioral states: A new approach to the assessment of psychosocial influences on ambulatory cardiovascular activity. In D. Krantz \& A. Baum (Eds.), Perspectives in Behavioral Medicine: Technology and Methodology in Behavioral Medicine (pp. 17-29). Hillsdale, NH: Lawrence Erlbaum.

Goodie, J. \& Kamarck, T. (1996). Use of the Accutracker DX to assess behavioral influences on ambulatory cardiovascular activity: A preliminary investigation. Blood Pressure Monitoring, 1, 135-140.

\section{MANUSCRIPTS SUBMITTED FOR PUBLICATION}

Kamarck, T. W., Raynor, D., Rutledge, T. Shiffman, S. M., Manuck, S. B., Debski, T. T., Kinder, L., Goodie, J. L., \& Thompson, H. (submitted 2001) Correspondence between laboratory and ambulatory measures of cardiovascular reactivity: A variance partitioning approach

\section{MANUSCRIPTS IN PREPARATION}

Joseph, K. A., Adams, C. A., \& Goodie, J. L. (in preparation). Treatment of panic disorder in an adolescent diagnosed with asthma: A case study. 
Goodie, J. L., \& Larkin, K. T. (in preparation). Prospective studies examining the relation between cardiovascular reactivity to stress and disorders of the cardiovascular system: A critical review.

Semenchuk, E., Larkin, K. T., \& Goodie, J. L. (in preparation). Coping behaviors and cardiovascular reactivity to stress.

\section{PUBLISHED ABSTRACTS AND PAPER PRESENTATIONS}

2001

Black, A., Edelstein, B., Martin, R., McKee, D., Goodie, J., Drozdick, L., \& Kalish, K. (July, 2001). Decision making across the lifespan: Preventive versus life-saving medical procedures. Paper presented at meeting of the World Congress of Behavioral and Cognitive Therapies. Vancouver, BC.

Black, A., L., Taylor, B. K., Goodie, J. L., \& Larkin, K. T. (March, 2001). Relation between home monitored blood pressure and mood state in a sample of hypertensive and normotensive individuals. Journal of Psychosomatic Medicine. Presented at the American Psychosomatic Society in Monteray, CA.

Goodie, J. L., Larkin, K. T., Taylor, B. K., Siegwarth, N., \& White, D. (March, 2001). An examination of the transfer of heart rate feedback training to reduce heart rate response to laboratory tasks. Journal of Psychosomatic Medicine,. Presented at the American Psychosomatic Society in Monteray, CA.

Goodie, J. L., Peterson, A. L., Talcott, G. W., Haddock, C. K., Dixon, C., Rugh, J., \& Wright, E. (March, 2001). State and trait anxiety in temporomandibular disorders (TMD): Relations with temporomandibular joint (TMJ) noise and facial pain. Presented at the Society for Behavioral Medicine Annual Meeting, in Seattle, WA.

\section{0}

Edelstein, B.A., McKee, D. R., Martin, R. R., Goodie, J. L., Black, A. L., Drozdick, L. W., Kalish, K. (November, 2000). The roles of experience and knowledge in medical decisionmaking. Presentation at the Gerontological Society of America.

Goodie, J. L., Zvolensky, M. J., Ruggiero, K. J., Black, A. B., Larkin, K. T., \& Taylor, B. K. (November, 2000). Predictors of emotional reactivity to laboratory challenges. Presented at the Association for the Advancement of Behavior Therapy.

Zvolensky, M. J., Goodie, J. L., McNeil, D. W., Sperry, J. A., \& Sorrell, J. T. (April, 2000). Anxiety sensitivity in the prediction of pain-related fear and anxiety in a heterogeneous 
chronic pain population. Presented at the Society for Behavioral Medicine Annual Meeting, in Nashville, TN.

1999

Boone, M., Drozdick, L., Wilson, C., Arias, R., Goodie, J., Federoff, L. \& Leach, S. (1999). Assessment of Adult-Residual ADD (ARADD) using neurocognitive and self-report measures. Archives of Clinical Neuropsychology, 14, 763-764. Presented at the National Academy of Neuropsychology Annual Meeting, in San Antonio, Texas.

Davig, J. P., Larkin, K. T., \& Goodie, J. L. (March, 1999). Does cardiovascular reactivity to stress measured in the laboratory generalize to thesis and dissertation meetings? Annals of Behavioral Medicine, 21, S135. Presented at the Society for Behavioral Medicine Annual Meeting, in San Diego CA.

Frazer, N. L., Larkin, K. T., Drozdick, L. W., Goodie, J. L., Aragona, B., \& Harburg, S. (March, 1999). The relation between family history of hypertension and cardiovascular reactivity to stress: Examination of covert and overt responses to stressors. Annals of Behavioral Medicine, 21, S139. Presented at the Society for Behavioral Medicine Annual Meeting, in San Diego CA.

Goodie, J. L. (November, 1999). Social relationship and health-related changes in older adults: Adaptations for assessment and therapy. Presented at the Association for the Advancement of Behavior Therapy Annual Meeting in Toronto, Ontario.

Goodie, J. L., Larkin, K. T., Black, A., \& Dailey, E. (November, 1999). Coping strategies associated with cardiovascular response reduction through heart rate feedback. Presented at the Association for the Advancement of Behavior Therapy Annual Meeting in Toronto, Ontario.

Goodie, J. L., Larkin, K. T., \& Dailey, E. (March, 1999). Changes in hemodynamic reactivity to a videogame challenge with heart rate feedback training. Annals of Behavioral Medicine, 21, S56. Presented at the Society for Behavioral Medicine Annual Meeting, in San Diego CA.

Goodie, J. L., Sperry, J. A., Sorrell, J. T., \& McNeil, D. W. (November, 1999). Anxiety sensitivity in a chronic pain population. Presented at the Association for the Advancement of Behavior Therapy Annual Meeting in Toronto, Ontario.

Goodie, J. L., Sperry-Clark, J. A., Ciano-Federoff, L. (March, 1999). The use of the PAI in a chronic pain population. Annals of Behavioral Medicine, 21, S56. Presented at the Society for Behavioral Medicine Annual Meeting, in San Diego CA.

Kamarck, T. W., Polk, D. E., Shiffman, S., Smithline, L., Thompson, H., Goodie, J., Paty, J., Kassel, J., Gnys, M. (March, 1999). Emotional support moderates the acute pressor 
effects of stress during daily life. Psychosomatic Medicine, 61, 112. Presented at the American Psychosomatic Society Annual Meeting, in Vancouver, B.C., Canada.

McKee, D. R., Goodie, J., Drozdick, L. W., Black, A., Martin, R., Kalish, K., \& Edelstein, B. A. (August, 1999). Medical decision-making regarding advance directives: The roles of heuristics and values. Presented at the American Psychological Association Annual Meeting, in Boston, MA.

1998

Drozdick, L. W., Goodie, J. L., James, J., \& Edelstein, B. (November, 1998). Relations between the AFABS and measures of executive and cognitive functioning. Poster presentation at the Gerontological Society of America in Philadelphia, PA.

Goodie, J. L. \& Kalish, K. (November, 1998). Functional assessment of nursing home residents. Presentation at the Association for the Advancement of Behavior Therapy Annual Meeting in Washington, DC.

Larkin, K. T., Schauss, S. L., Elnicki, D. M., Goodie, J. L. (March, 1998). Clinical indicators of "White Coat" hypertension. Annals of Behavioral Medicine, 20, S89. Presented at the Society for Behavioral Medicine Annual Meeting, in New Orleans, LA.

1997

Ciano-Federoff, L. M., Larkin, K. T., \& Goodie, J. L. (March, 1997). Coping mechanisms as partial mediators between depression and health status. Paper presented at the Society of Behavioral Medicine Annual Meeting, in San Francisco, CA.

Goodie, J. L., Schauss, S., Larkin, K. T., Aragona, B. (October, 1997). Validation of the Polar heart rate monitor for measuring heart rate responses to mental stress. Psychophysiology, 34, S38. Presented at the Society for Psychophysiological Research Annual Meeting, in Cape Cod, MA.

Gump, B., Kamarck, T., Shiffman, S., Smithline, L., Thompson, H., Goodie, J., Pro, V., Paty, J., Kassel, J., \& Gnys, M. (March, 1997). Marital satisfaction and cardiovascular reactivity: Looking within the home. Paper presented at the Society of Behavioral Medicine Annual Meeting, in San Francisco, CA.

Kamarck, T. W., Shiffman, S. S., Smithline, L., Thompson, H., Goodie, J., Paty, Gnys, M., \& Kassel, J. (October, 1997). The effects of psychosocial influences on ambulatory blood pressure: Contrasting different measurement and data analytic strategies. Psychophysiology, 34, S6. Presentation at the Society for Psychophysiological Research Annual Meeting, in Cape Cod, MA.

Raynor, D. A., Kamarck, T. W., Shiffman, S. S., Ituarte, P., Thompson, H., Smithline, L., Goodie, J., Paty, J., Gnys, M., \& Kassel, J. (October, 1997). The effects of social influence on 
cardiovascular responsiveness in the natural environment. Psychophysiology, 34, S73. Presented at the Society for Psychophysiological Research Annual Meeting, in Cape Cod, MA.

Smithline, L., Kamarck, T., Shiffman, S., Thompson, H., Goodie, J., Pro, V., Paty, J., Kassel, J., \& Gnys, M. (March, 1997). The effects of hostility on social conflict and cardiovascular reactivity during daily life. Paper presented at the Society of Behavioral Medicine Annual Meeting, in San Francisco, CA.

Smithline, L., Kamarck, T. W., Shiffman, S. S., Thompson, H., Goodie, J., Paty, J., Gnys, M., \& Kassel, J. (October, 1997). Job strain and ambulatory blood pressure in a healthy community sample: Failure to replicate. Psychophysiology, 34, S82. Paper presented at the Society for Psychophysiological Research Annual Meeting, in Cape Cod, MA.

\section{6}

Davig, J. P, Larkin, K. T., \& Goodie J. L. (November, 1996). Does cardiovascular reactivity to stress measured in the laboratory generalize to thesis and dissertation meetings? Paper presented at the Association for the Advancement of Behavior Therapy Annual Meeting in New York, NY.

Ituarte, P., Kamarck, T. W., Shiffman, S., Goodie, J., Smithline, L., Pro, V., Gnuys, M., Paty, J. \& Kassel, J. (March, 1996). Received support buffers the effects of daily stressors on well-being: An ecological momentary assessment approach. Annals of Behavioral Medicine, S92. Paper presented at the Society of Behavioral Medicine Annual Meeting, in Washington, DC.

Kamarck, T. W., Shiffman, S., Manuck, S. B., Debski, T. T., Cerrone, P., Smithline, L., Goodie, J. Pro, V., Gnys, M., Paty, J., \& Kassel, J. (March, 1996). Correspondence between laboratory and ambulatory measures of cardiovascular reactivity. Annals of Behavioral Medicine, S92. Paper presented at the Society of Behavioral Medicine Annual Meeting, in Washington, DC.

Kamarck, T. W., Shiffman, S., Smithline, L., Goodie, J. Pro, V., Paty, J., \& Kassel, J. (March, 1996). The effects of social behavior on ambulatory cardiovascular activity. Annals of Behavioral Medicine, S95. Paper presented at the Society of Behavioral Medicine Annual Meeting, in Washington, DC.

\section{4}

Goodie, J. \& Kamarck, T. (October, 1994). Validation of the Accutracker DX ambulatory blood pressure monitor. Psychophysiology, 31, S52. Paper presented at the Society for Psychophysiological Research Annual Meeting, in Atlanta, GA. 


\section{EDITORIAL ACTIVITIES}

\section{Reviewer}

2000

Journal of Psychophysiology

1999

Journal of Psychopathology and Behavioral Assessment

Student Ad Hoc Editorial Reviewer with:

2000

Barry Edelstein, Ph.D.

The Journal of Gerontology: Psychological Science

1999

Kevin Larkin, Ph.D.

Journal of Psychosomatic Research

1999

Barry Edelstein, Ph.D.

Psychological Assessment

1998

Kevin Larkin, Ph.D.

Psychosomatic Medicine

1997

Kevin Larkin, Ph.D.

Journal of Behavioral Medicine

MEMBERSHIP IN PROFESSIONAL ORGANIZATIONS

Student Membership American Psychological Association

Association for the Advancement of Behavior Therapy

Society for Behavioral Medicine

Society for Psychophysiological Research 